

\section{A STUDY OF LOW ENERGY GALACTIC COSMIC RAYS \\ FROM 1961 TO 1965}

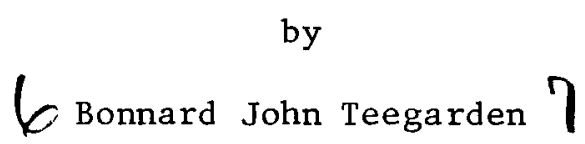

Thesis submitted to the Faculty of the Graduate School of the University of Maryland in partial fulfillment of the requirements for the degree of Doctor of Philosophy 1967 


\section{ABSTRACT}

Title of thesis: A Study of Low Energy Galactic Cosmic Rays from 1961 to 1965.

Bonnard Teegarden, Doctor of Philosophy, 1967

Thesis directed by: Frank B. McDonald

Visiting Professor

The results from a series of balloon flights beginning in 1961 and ending in 1965 are presented. Measurements of the cosmic ray intensity were made using $a \mathrm{dE} / \mathrm{dx}$ and $\mathrm{E}$ detector sensitive to energies from 15 to $80 \mathrm{MeV} /$ nucleon. The early balloon flights provided design information and also aided in the development of data handing techniques for later satellite versions of the detector which have been on IMP's I, II, and III and OGO's I and II. Proton and helium intensities at $85 \mathrm{MeV} /$ nucleon are presented for the five year period covered by the balloon flight series. The behavior of the proton to helium ratio as a function of time is discussed within the framework of Parker's model for the solar modulation of cosmic rays.

In 1965 a modified version of the $\mathrm{dE} / \mathrm{dx}$ and $\mathrm{E}$ detector with an extended energy range was flown for the first time. A cosmic ray helium spectrum from 60 to $500 \mathrm{MeV} /$ nucleon measured by this detector is presented. The change in proton and helium intensities in this energy region from 1963 to 1965 is examined and compared with the results predicted by the various special cases of Parker's model.

A totally empirical atmospheric secondary proton spectrum is derived, based on simultaneous balloon and satellite measurements. This spectrum is compared with the secondary spectrum obtained from a nuclear emulsion measurement and the differences are discussed. Using our empirical 
secondary spectrum, we obtain an upper limit for the re-entrant albedo at Sioux Falls which is significantly less than values reported by other observers.

A measurement of the intensities of secondary deuterium and tritium was made in 1965 . Using these results, we obtain a value for the global average production of tritium in the earth's atmosphere. The implications of this result regarding the problem of tritium balance in the atmosphere are discussed. 


\section{ACKNOWLEDGEMENTS}

The author wishes to express his deep gratitude for the guidance and encouragement of his thesis advisor, Dr. F. B. McDonald.

Special thanks are due to Dr. D. A. Bryant whose work during the early stages of the experimental program was of great value.

Enlightening discussions with Drs. V. K. Balasubrahmanyan, K. A. Brunstein, U. D. Desai, and D. E. Guss were particularly useful. Many people contributed to the design and construction of the experiment. The author wishes to particularly acknowledge the contributions of Mssrs. C. A. Cancro, F. Clese, A. P. Flanick, and R. G. McGowan.

The typing was ably done by Mrs. S. L. Premeaux. 
Chapter

ACKNOWLEDGMENTS . . . . . . . . . . . . . . . . . . ii

LIST OF FIGURES . . . . . . . . . . . . . . . . . . . . . iv

LIST OF TABLES . . . . . . . . . . . . . . . . . . . . . vi

I. INTRODUCTION •. . . . . . . . . . . . . . . . 1

II. EXPERIMENTAL PROCEDURE . . . . . . . . . . . . . . 15

A. The $\mathrm{dE} / \mathrm{dx}$ and $\mathrm{E}$ Technique ............ 15

B. The Design of the Detector . . . . . . . . . . 17

C. The Flight Circuitry . . . . . . . . . . . . 18

D. The Modified Detector . . . . . . . . . . 19

III. THE BALLOON FLIGHT SERIES . • • • • . . • . • • . • 25

IV. DATA ANALYSIS. . . . . . . . . . . . . . . . . . 29

A. Stopping Particle Analysis . . . . . . . . . . 29

B. Penetrating Particle Analysis . . . . . . . . 35

V. TIME VARIATIONS OF THE LOW ENERGY PROTON AND ALPHA PARTICLE

INTENSITIES . . . . . . • . • • • • • • • • 39

A. General Remarks ............... . 39

B. The Cosmic Ray Intensity at $85 \mathrm{MeV} /$ nucleon from 1961 to 1965 . . . . . . . . . . . . . . . . 44

C. Solar Modulation in the 80-500 MeV/nucleon Interval

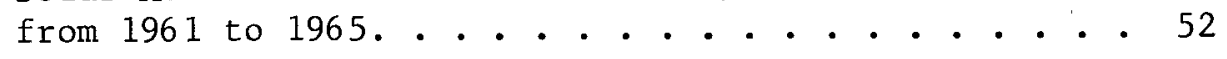

VI. ATMOSPHERIC SECONDARIES AND RE-ENTRANT ALBEDO • • • • • . 57

A. Atmospheric Secondaries . . . . . . . . . 57

B. Re-entrant Albedo ................. . . 64

VII. PRODUCTION OF DEUTERIUM AND TRITIUM IN THE EARTH's ATMOSPHERE . . . . . . . . . . . . . . . . 66

VIII. CONCLUDING REMARKS . • . • • • • . . . . . . • 76 REFERENCES . . . . . . . . . . . . . . . . . . . 108 


\section{LIST OF FIGURES}

Figure

Page

1. TOTAL COSMIC RAY SPECTRUM AT SOLAR MINIMUM . . . . . . . . 78

2. COSMIC RAY RELATIVE ABUNDANCES . . . . . . . . . . . . 79

3. IDEALIZED VIEW OF THE DE/DX AND E DETECTOR . . . . . . . . 80

4. $\Delta \mathrm{E}$ VS, $\mathrm{E}-\Delta \mathrm{E}$. . . . . . . . . . . . . . . . . 81

5. DE/DX AND E DETECTOR DIAGRAM . . . . . . . . . . . . . . 82

6. BLOCK DIAGRAM - MARK II CIRCUITRY . . . . . . . . . . 83

7. IDEALIZED VIEW OF THE MODIFIED DE/DX AND E DETECTOR • • . 84

8. $\triangle$ E VS. E- $\triangle E$ FOR PENETRATING PARTICLES . . . . . . . . . . . 85

9. BLOCK DLAGRAM - MARK VII CIRCUITRY . . . . . . . . . . . 86

10. SCHEMATIC DIAGRAM - PULSE RISE-TIME DISCRIMINATOR • • • . 87

11. Alt ITUde CURVE; FLIGHT $62-3 \ldots \ldots$. . . . . . . . . . . . . 88

\begin{tabular}{l} 
12. SMOOTHED SUNSPOT NUMBER AND MT. WASHINGTON NEUTRON MONITOR \\
RATE... \\
\hline
\end{tabular}

13. $\triangle \mathrm{E}$ VS. E- $\triangle \mathrm{E}$ (STOPPING PARTICLES); FLIGHT 65-1; $128 \times 128$ CHANNELS . . . . . . . . . . . . . . . . . 90

14. $\triangle E$ VS. E- $\triangle E$ (STOPPING PARTICLES); FLIGHT 65-1; $512 \times 512$ CHANNELS . . . . . . . . . . . . . . . . 91

15. PROTON MASS HISTOGRAMS: FLIGHT 65-1 . . . . . . . . . . . 92

16. $\triangle E$ VS. E- $\Delta$ E (PENETRATING PARTICLES); FLIGHT 65-1; $128 \times 128$ CHANNELS . . . . . . . . . . . . . . . . . . 93

17. $\Delta$ E VS. E- $\Delta$ E (PENETRATING PARTICLES); FLIGHT 65-1; $128 \times 128$ CHANNELS; SELECTION CRITERIA IMPOSED . . . . . . . . . . . 94

18. $\Delta$ E VS. E-AE (PENET RATING PART ICLES); FLIGHT 65-1; $512 \times 512$ CHANNELS; SELECTION CRITERIA IMPOSED . . . . . . . . . . . 95

19 CROSS - SECTIONS OF THE PENETRATING HELIUM LINE . • • • • 96

20. TIME VARIATIONS DURING FLIGHT 63-1 . . . . . . . . . . . 97

21. PROTON AND HELIUM INTENSITIES AT $85 \mathrm{MEV} /$ NUCLEON...$\quad$. . 98

22. PROTON TO HELIUM RATIO AT 85 MEV/NUCLEON . . . . . . . . . 99 
Figure $\quad$ Page

23. 1965 HELIUM SPECTRUM • . . . . . . . . . . . . . . . . 100

24. RATIO OF 1963 AND 1965 INTENSITIES . . . . . . . . . . . . 101

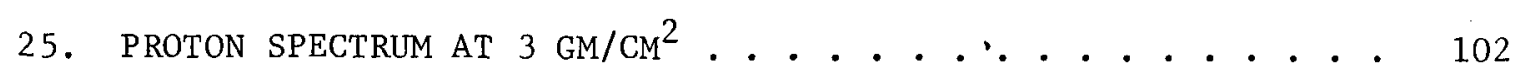

26. SECONDARY PROTON INTENSITY VS. DEPTH IN ATMOSPHERE . . . . . 103

27. COMPARISON OF SECONDARY SPECTRUMS . . . . . . . . . . . . . 104

28. RE-ENTRANT ALBEDO AT SIOUX FALLS (5 $\mathrm{GM}^{\left.-\mathrm{CM}^{2}\right)}$. . . . . . • . 105

29. DEUTERIUM AND TRITIUM MASS HISTOGRAMS . . • . . • . . . • . . 106

30. SECONDARY PROTON AND DEUTERON ALTITUDE DEPENDENCE . . . . • . 107 
Table

Page

I. FLIGHT DATA . . . . . . . . . . . . . . . . . 26

II. SUMMARY OF INTENSITIES AT FORT CHURCHILL . . . . . . . . . . . 40

III. HYDROGEN ISOTOPE PRODUCTION . . . . . . . . . . . . . 70 


\section{INTRODUCTION}

In recent years cosmic rays have become an increasingly important tool for studying problems of astrophysical interest. Classical astronomy and astrophysics have traditiona1ly relied upon studies of stellar electromagnetic radiation in the visible and, more recently, in the radio region. Cosmic rays, consisting of energetic charged particles, constitute an entirely different type of radiation. The corpuscular cosmic radiation is therefore a potential source of new information not obtainable from the more familiar methods of optical and radio astronomy.

Since cosmic rays consist of charged particles, they will, unlike photons, interact with the interplanetary and galactic magnetic fields. An important facet of cosmic ray research concerns the influence of such magnetic fields on the propagation and distribution of cosmic rays in the solar system and in our galaxy. Evidence exists that there is at least an approximate equipartition of energy between the galactic magnetic field and galactic cosmic rays. Each has an energy density of approximately $1 \mathrm{eV} / \mathrm{cm}^{3}$. Such an equipartition suggests that cosmic rays may play an important role in magnetohydrodynamical processes within our galaxy.

We now ask what are the observable properties of galactic cosmic rays, and what, specifically, can be learned from each observation? The observations can be divided into four categories:

1. The energy spectrum.

2. Nuclear and isotopic abundances.

3. Time variations.

4. Arrival directions. 
These observational categories are not independent since, for example, the time variations and isotopic composition are both energy dependent.

The total cosmic ray kinetic energy spectrum at solar minimum is shown in Figure 1. This is necessarily a compilation of many different experimental results, since no single experiment could span such a wide range of energy. Cosmic ray measurements have extended over approximately 14 orders of magnitude in energy. Air shower experiments have measured cosmic ray energies of $\sim 10^{20} \mathrm{eV}$, the largest single particle energy ever recorded.

We see in Figure 1 that the energy spectrum undergoes a maximum in the vicinity of $500 \mathrm{MeV}$. At present it is not clear whether this is a property of primary cosmic rays or a local effect caused by the solar modulation of galactic cosmic rays. At higher energies where solar modulation has little or no influence, the cosmic ray spectrum exhibits a power law dependency $\left(\mathrm{dj} / \mathrm{dE} \propto \mathrm{E}^{-\mathrm{n}} ; \mathrm{n} \cong 2.5\right)$. This behavior persists over an extremely wide range in energy, approximately ten orders of magnitude. Evidence exists, however, indicating the presence of some fine structure in the spectrum at very high energies $\left(\gtrsim 10^{16} \mathrm{eV}\right)$. Such a fine structure is related to the problem of containment of cosmic rays within our galaxy and also to the question of whether cosmic rays are present in intergalactic as well as interstellar space.

The composition of galactic cosmic rays is summarized in Figure 2 . It is interesting to compare the relative abundances of galactic cosmic rays with the so-called cosmical abundances as determined from meteorites and stellar spectrums. Qualitatively, the cosmic ray and cosmical abundances exhibit similar behaviors. In both cases hydrogen is overwhelmingly the 
single most abundant element, followed by helium, carbon, and oxygen. In addition, an odd-even effect $c$ an be found in both the cosmic ray and cosmical abundances, whereby nuclei of even $\mathrm{Z}$ are in general more abundant than nuclei of odd $z$. An anomalously high abundance of heavy galactic cosmic rays, particularly in the iron, cobalt, nickel group, has been observed compared with the cosmical abundances. The cosmical abundances are, of course, representative of average or typical conditions. The anomalously high abundance of the heavy elements in the galactic cosmic radiation suggests that the acceleration of cosmic rays takes place in an atypical region such as might be found, for example, in a supernova. An anomalously high abundance in the galactic cosmic radiation has also been observed for the light nuclei lithium, beryllium, and boron. It is believed that such nuclei are produced by the fragmentation of heavier nuclei during their passage through interstellar material. From measurements of the relative abundances of the light nuclei, it has been deduced that cosmic rays, on the average, pass through approximately $3 \mathrm{gm} / \mathrm{cm}^{2}$ of interstellar material.

Cosmic ray time variations have been the subject of much research ir recent years. Several different types of short term time variations (lasting from a few hours to several weeks) have been observed. These are the diurnal and semi-diurnal variations, the Forbush decrease, and the 27 day variation. The short-term time variations are, in genera1, related to the spatial structure and to time variations of the interplanetary magnetic field.

The anti-correlation between the long-term time variation in the galactic cosmic ray intensity and the 11 year sunspot cycle was first noted by Forbush [1954]. The eleven year variation in cosmic ray 
intensity is attributed to modulation of the galactic cosmic ray intensity by the solar magnetic field. High latitude neutron monitors, sensitive to average energies of 3-5 $\mathrm{BeV}$, characteristically show variations in intensity of $30 \%$ over a solar cycle.

Cosmic ray arrival directions are very nearly isotropic. Isotropy is, in fact, expected as a result of scattering by irregularities in the galactic magnetic field. Careful observations, however, have shown that a sma11 quiescent anisotropy $(\sim .5 \%)$ exists, with a maximum at approximately $90^{\circ}$ to the earth-sun line. This anisotropy manifests itself in the form of the diurnal variation mentioned previously. Its magnitude and direction are consistent with the hypothesis that the cosmic ray gas is swept along with the interplanetary magnetic field which, in turn, is co-rotating with the sun. In addition, larger, but short-lived, anisotropies have been observed in conjunction with Forbush decreases.

In the context of this thesis we shall be concerned only with the low energy cosmic rays $(\mathrm{E} \lesssim 500 \mathrm{MeV} /$ nucleon). The low energy cosmic rays are much more susceptible to the influence of interplanetary and galactic magnetic fields. Solar modulation is consequently much more effective at low energies than at high energies. The proton intensity at $85 \mathrm{MeV}$, which will be discussed more fully later, has been observed to change by a factor of four from 1961 to 1965; therefore the change between solar maximum and solar minimum is expected to be even larger. This is to be compared with a change of only $30 \%$ in the intensity at $4 \mathrm{BeV}$ from solar maximum to solar minimum as indicated by sea-level neutron monitors. Studies of the time variation at low energies are thus particularly useful in attempting to understand the process of solar modulation. 
The behavior of the galactic cosmic ray spectrum at low energies is as yet an unsolved problem. One hopes that with a better understanding of solar modulation it will be possible to demodulate the spectrum observed at the earth's orbit and thus obtain a galactic cosmic ray spectrum. One expects that effects due to ionization losses occurring during passage through the interstellar gas will become important at low energies. Thus, a knowledge of the behavior of the low energy portion of the cosmic ray spectrum should improve the understanding of the acceleration and propagation of cosmic rays within our galaxy. In addition, the low energy region is a particularly fertile area for the study of the isotopic composition of cosmic rays. While charge identification is easy at high energies, mass separation is quite difficult. At low energies, however, it is possible in certain cases to separate isotopes of a single element. Studies have been made, for example, of the $\mathrm{He}^{3} / \mathrm{He}^{4}$ ratio in the low energy region. Early measurements presented a somewhat confusing picture of the low energy portion on the cosmic ray spectrum. High altitude latitude surveys [see, for example; Meredith, Van Allen, and Gottlieb, 1955; Meyer and Simpson, 1955, 1957; Winckler and Anderson, 1957] provided the first experimental evidence relating to the behavior of low energy cosmic rays. A "knee" or plateau in the latitude curve was observed: the intensity as a function of latitude increased up to a certain point and thereafter remained constant. The knee was not always present; nor was its position always the same, but seemed to be correlated with solar activity. The early experimenters were confronted with the problem of whether the knee really reflected the absence of low energy particles or was simply due to atmospheric absorption. Unfortunately, incorrect theoretical estimates of the geomagnetic cut-offs at high latitudes led in many cases to a 
misinterpretation of the data. Certain latitude surveys were interpreted as indicating the absence or extreme scarcity of cosmic rays below energies as high as 500 to $600 \mathrm{MeV}$. Later, more direct measurements showed that, in fact, the knee in the latitude curve is due to atmospheric absorption of the low energy primary cosmic rays.

Additional evidence on the flux at low energies was obtained in the early 1950's through studies of the ionization rate as a function of depth in the atmosphere [see, for example; Neher, 1957]. The ionization rate characteristically exhibits an exponential dependence on depth in the atmosphere. However, Neher found a "turning up" in the ionization vs. depth curve at high altitudes $\left(<10 \mathrm{gm} / \mathrm{cm}^{2}\right)$ near the geomagnetic pole at solar minimum. This behavior was attributed to particles with ranges in the neighborhood of $10 \mathrm{gm} / \mathrm{cm}^{2}$, specifically, protons with energies of approximately $100 \mathrm{MeV}$. A correlation was found between the shape of the ionization vs. depth curve and solar activity, and some speculation ensued concerning the relation of the "knee" to the ionization-depth curve. The first indication of the strong influence of solar modulation at low energies was obtained from these studies. Data on the latitude and depth dependence of the ionization rate, however, cannot provide detailed information on the behavior of low energy cosmic rays.

Nuclear emulsions provided one of the earliest means of obtaining direct measurements of the cosmic ray spectrum at low energies. The phrase "direct observations" should perhaps be somewhat qualified since the measurements are contaminated by secondary protons and deuterons produced in the atmosphere above the detector, and by re-entrant albedo. Emulsions provide a quite versatile method for charged particle detection. 
A number of different techniques permit identification of a particle's charge, mass and energy; but careful and lengthy analysis is necessary to yield enough data to determine a statistically significant spectrum. Primary cosmic ray studies by means of nuclear emulsions began around 1948 and continue through the present. Comprehensive summaries of the early work have been presented by Singer [1958] and Waddington [1960]. More recent emulsion are summarized in several review papers [Webber, 1962, 1964; Biswas and Fichte1, 1965].

The early emulsion measurements provided the first evidence on the composition of cosmic rays. The anomalously high abundance of heavy nuclei mentioned previously was first discovered with emulsions. A large abundance of the light elements as compared to universal abundances was also observed. Initially there was some ambiguity as to whether these were really primary particles or just fragmentation products from interactions in the atmosphere. Later experiments, however, have shown that these particles are, in fact, present in the primary cosmic radiation. Except for a few cases, the early emulsion experiments relied on the latitude effect to magnetically analyze the cosmic ray spectrum. Values for the integral intensities of the various cosmic ray components were obtained at different latitudes. This technique, however, was not we11 suited to the study of the low energy cosmic ray spectrum.

The measurements of Waddington [1954] provided one of the first direct determinations of the differential cosmic ray spectrum at low energies: the alpha particle spectrum was obtained down to $400 \mathrm{MeV} /$ nucleon. Above $400 \mathrm{MeV} / \mathrm{nucleon}$, this spectrum was observed to decrease monotonically towards higher energies. Then the emulsion measurements of Ney [1955] 
extended the differential alpha spectrum down to $150 \mathrm{MeV} /$ nucleon: a maximum was observed at approximately $300 \mathrm{MeV} /$ nucleon, and thereafter the spectrum decreased towards lower energies.

A number of emulsion measurements of the differential spectrum at low energies were made in the late 1950 's [see for example, Duke, 1960; Freier et al., 1959; Fowler et a1., 1958]. With these measurements in addition to the Cerenkov detector measurements of McDonald and Webber, a fairly consistent picture of the low energy spectrum began to emerge.

The Cerenkov-scintillator telescope of McDonald and Webber provided an extremely useful tool for the measurement of cosmic rays in the 300$800 \mathrm{MeV} /$ nucleon range. The combined Cerenkov and scintillator output gives a unique determination of a particle's charge and velocity. An extensive series of balloon flights was carried out by McDonald and Webber during the middle and late 1950's [Webber and McDonald, 1955, 1964; McDonald and Webber, 1956, 1957, 1959, 1962].

McDonald and Webber's first measurements of the alpha particle spectrum were in essential agreement with emulsion measurements. In later balloon flights the proton spectrum was measured between 250 and $750 \mathrm{MeV}$ [McDonald and Webber, 1959]. One of the most significant aspects of the data was that the differential rigidity spectra of the alpha particles and protons were related by a simple constant of proportionality. This led to the conclusion that the mechanism producing the shape of the spectrum in this region, whether it be primary acceleration, solar modulation, or a combination of both, must be rigidity dependent. It was a reasonable conclusion, since acceleration and modulation mechanisms due to magnetic fields characteristically have particle rigidity as a fundamental para- 
meter. One must remember, however, that the above observations will be strongly dependent on the solar cycle. The proton spectrum measurement of McDonald and Webber was made near maximum solar activity and, consequently, the conclusions may not necessarily be valid during more quiet periods. Also, the region of overlap of the proton and alpha rigidity spectra was not large and did not really preclude a splitting of the spectra. In fact, certain more recent observations near solar minimum appear to indicate a velocity-dependent modulation mechanism at low energies.

Before 1960 the cosmic ray spectrum had been measured down to an energy of approximately $250 \mathrm{MeV}$ for protons and $150 \mathrm{MeV} / \mathrm{nucleon}$ for alphas. Interest naturally arose in the spectrum at still lower energies since it appeared to depend more strongly on solar modulation and was potentially a new source of information on astrophysical processes within the galaxy. The measurements of Meyer and Vogt in the early 1960's [Vogt, 1962; Meyer and Vogt, 1963] were the first to provide information in this energy region. Their experimental technique involved the simultaneous measurement of a particle's energy loss rate $(\mathrm{dE} / \mathrm{dx})$ and its range. The range was measured by using alternate layers of lead absorber and scintillator; this combination provided a unique identification of a particle's charge, mass, and energy, and was able to detect protons in the 80-300 MeV interval. Thus, unlike McDonald and Webber's experiment, it distinguished electrons from protons. Alphas and heavier nuclei, however, were difficult to detect because of nuclear interactions in the large thickness of absorber.

The primary proton spectrum observed by Vogt in 1960 appeared to increase towards lower energies below about $250 \mathrm{MeV}$. The high abundance 
of low energy protons and their spectral behavior led to speculation that at least during periods of high solar activity, the sun might continuously emit energetic particles. Interpretation of the data of Vogt [1962] and Meyer and Vogt [1963] is complicated by atmospheric secondaries which become particularly important at very low energies. Analysis is also complicated by nuclear interactions within the detector itself. Furthermore, small solar cosmic ray events, when they occur, would dominate the low energy region.

Later balloon and satellite measurements [see, for example Brunstein, 1964; Fichte1, et al., 1964; McDonald and Ludwig, 1964] have not found the large number of low energy protons reported by Meyer and Vogt. This does not, however, preclude the possibility that these were present during the time of Vogt's measurement (1960) and not present during later periods of less solar activity. The data to be presented in this paper have some bearing on this question, as will be discussed later.

Emulsion and counter measurements of the low energy spectrum have continued through the declining solar cycle. Detailed information on the time variation as a function of energy of proton and alpha particle intensities has been obtained. The advancement of satellite technology has permitted direct measurements uncomplicated by albedo and atmospheric secondaries.

A detector aboard the eccentric orbiting Explorers XII and XIV provided detailed information on low energy solar cosmic rays [Bryant, et a1., 1962, 1962, 1965]. Ion chambers on deep space probes have observed the gross temporal and spatial behavior of cosmic rays [see, for example, Neher and Anderson, 1964; Anderson, 1963, 1964]. 
The first direct measurements of galactic cosmic rays below 100 MeV were made by detectors aboard the IMP-I satel1ite in 1963. The detectors were of the $\mathrm{dE} / \mathrm{d} \mathbf{x}$ and $E$ type and were similar in principle to that used by Meyer and Vogt. Instead of measuring a particle's range, however, a total absorption scintillator was used to measure its energy. This technique has the advantage of providing a continuous measurement of a particle's total energy as opposed to determining in which range interval (out of perhaps 6 or 7) a particle falls. Background produced within the detector can be eliminated more easily and, as a result, particles of much higher charge and mass can be measured. Proton and alpha particle intensities were obtained between approximately 30 and $80 \mathrm{MeV} /$ nucleon [Fan, et a1., 1964, 1965a, 1965b; Gleockler, 1965; McDonald and Ludwig, 1964; Balasubrahmanyan, et a1., 1965]. There was general agreement between the satellite measurements and balloon measurements at comparable and higher energies carried out during the same period of time [see, for example, Ormes and Webber, 1963; Freier and Waddington, 1964]. The observed spectra exhibited no surprising characteristics. The low energy portion showed a maximum at about $300 \mathrm{MeV} /$ nucleon and then decreased monotonically down to the lowest measured energies of approximately 20 MeV/nucleon. Identical detectors were installed on IMP's II and III, and on OGO I. Through the combined balloon and satellite observations, statistically significant detailed information on the behavior of the cosmic ray charge and energy spectrum has thus been obtained.

The improved quality of the data has permitted a more detailed analysis of the solar modulation mechanism. A number of theoretical models for the solar modulation have been presented. Parker's mode1, 
involving diffusion and outward convection of galactic cosmic rays by irregularities in the solar magnetic field, has perhaps received the most attention. The simplest form of the model predicts a velocity dependent modulation, at least at lower energies where the scale size of the magnetic irregularities exceeds the particles' radius of gyration in the solar field. In fact, the velocity spectra of protons and alphas at low energies appear to be identical when appropriately normalized. A more detailed analysis by Gloeckler [1965] of the time variation of the alpha particle spectrum revealed a velocity dependent modulation below $100 \mathrm{MeV} /$ nucleon. The Goddard group [Balasubrahmanyan, et a1., 1966] has reported agreement with Parker's model, using the thick scattering center approximation, up to approximately $1 \mathrm{BeV}$. Many aspects of the recent data are still not fully understood. At present, sufficiently accurate data exists covering a period of only two or three years near solar minimum. One cannot, therefore, be certain that the conclusions drawn will be valid over the entire solar cycle. The continuation of similar observations up to and beyond the next solar maximum is therefore of primary importance.

As was stated earlier, the low energy cosmic rays are particularly sensitive to solar modulation. With this in mind a detector designed to measure the intensity of cosmic rays in the 20-80 MeV interval was flown in a series of balloon flights beginning in 1961 and ending in 1965, thus providing a monitor of the low energy cosmic ray intensity over a major portion of the solar cycle. The early balloon flights also provided design data for the later satellite flights.

The detector is of the $\mathrm{dE} / \mathrm{dx}$ and $E$ type and is essentially identical 
to that used by McDonald and co-workers on the IMP and OGO satellites. The satellite version of the detector, of course, has inherent advantages over a balloon-borne experiment in that it is free from background due to secondaries produced in the atmosphere above the balloon. However, the first satellite, IMP I, carrying this experiment was not launched until November, 1963. Consequently, the balloon data, which cover a larger portion of the solar cycle are expected to provide more information on long-term time variations than at present can be obtained from the satellite data.

While specifically designed to provide monitor the low energy primary cosmic ray intensity, the series of balloon flight experiments also yielded information on other areas of interest. In addition to the data on low energy primaries we shall present in the following chapters data pertaining to the re-entrant albedo and to the production of secondary protons, deuterons, and tritons in the earth's atmosphere. Specifically:

1. Data will be presented on the time variation of the proton and helium intensities at $85 \mathrm{MeV} /$ nucleon from 1961 to 1965. We shall show that the low energy intensity is indeed anti-correlated with solar activity, and that there does not appear to be any quiescent solar particle contribution at least as far back as 1961 .

2. In order to extend the energy response a new version of the detector was developed and flown for the first time in 1965 . We shall present a cosmic ray alpha particle spectrum measured by this detector in 1965 . The fractional change in intensity in the 80 to $500 \mathrm{MeV} /$ nucleon interval from 1963 to 1965 will be examined and compared with the predictions of the various special cases of Parker's model of the solar modulation. We 
shall show that no single case appears to provide a consistent fit to all the data.

3. We shall derive a completely empirical atmospheric secondary spectrum based on simultaneous balloon and satellite measurements using the same detector of the low energy intensity. As part of the balloon flight series, several flights were made at lower latitudes where the geomagnetic cutoff is well above the maximum energy acceptable by the detector. Using the results from these flights combined with our empirical atmospheric secondary spectrum, we will place an upper bound on the re-entrant albedo intensity that is significantly smaller than the absolute intensities given by other experimentors.

4. Finally, data on the production of deuterium and tritium in the earth's atmosphere will be presented. Our experimental result for the tritium production rate will be compared with the theoretical results of other workers, and the question of the tritium balance in the earth's atmosphere will be discussed. 


\section{EXPERIMENTAL PROCEDURE}

A. The $d E / d x$ and E Technique.

We now ask, what requirements must be met by an experiment intended to measure the intensity and composition of low energy cosmic rays?

Under the most favorable conditions - i.e. at solar minimum - the cosmic ray nuclei with energies less than $100 \mathrm{MeV} /$ nucleon comprise less than $5 \%$ of the total cosmic radiation. We must therefore construct a detector capable of identifying these low energy particles in the presence of a much more intense and higher energy background radiation.

In nearly al1 experiments designed to measure low energy intensities, the incident particle loses part or al1 of its energy by ionization in the detector. This energy loss is then measured by a variety of techniques; e.g., light emission from a scintillator, or a current pulse from a solid state detector. It is possible, however, for a particle to lose energy by a nuclear interaction within the detector; and a higher energy particle producing such a spurious event could be incorrectly identified as a low energy particle. This problem becomes important when the high energy intensity is much larger than the low energy intensity.

The measurement of a single parameter (e.g. energy, energy loss rate, range) is not sufficient to determine the charge and mass of a low energy particle. For example, a slow proton might have the same energy loss rate as a fast alpha particle. In general, two or more measurements are required. Multiparameter analysis has the additional advantage of permitting discrimination against the aforementioned spurious events. In the detector developed for this work, two parameters are measured: the particle's energy loss rate, $d E / d x$, and its total energy, E. The $d E / d x$ and $E$ detector was first developed for use in nuclear physics as a convenient method for doing 
nuclear spectroscopy when dealing with different species of particles. The energy loss rate can be expressed in the form $d E / d x=z^{2} f(v)=$ $\mathrm{Z}^{2} \mathrm{~g}(\mathrm{E} / \mathrm{M})$ where $\mathrm{E}$ is the particle's incident energy, $\mathrm{v}$, its velocity, and $M$ its mass. The function $g(E / M)$ can be written $g(E / M)=g_{1}(M) g_{2}(E)$ and the energy loss rate becomes $\mathrm{dE} / \mathrm{dx}=\left[\mathrm{Z}^{2} \mathrm{~g}_{1}(\mathrm{M})\right] \mathrm{g}_{2}(\mathrm{E})$. Therefore the simultaneous measurement of $\mathrm{dE} / \mathrm{dx}$ and $\mathrm{E}$ determines the value of $\mathrm{z}^{2} \mathrm{~g}_{1}(\mathrm{M})$. For the particles of interest here, the value of $\mathrm{z}^{2} \mathrm{~g}_{1}(\mathrm{M})$ uniquely determines the specie of the particle.

Figure 3 is an idealized drawing of the detector. The $d E / d x$ measurement is accomplished by the thin upper scintillator. It is then possible to select those events in which a particle stops in the thick lower scintillator by demanding an anti-coincidence with the plastic guard scintillator C. For an infinitely thin upper scintillator of thickness $\Delta x$ the energy deposition is simply $\Delta E=\frac{\mathrm{dE}}{\mathrm{d} x} \Delta \mathrm{x}$, and the energy deposition in the lower scintillator is $\mathrm{E}-\Delta \mathrm{E}=\mathrm{E}$ since $\Delta \mathrm{E}$ is infinitely sma11. The upper or $\Delta \mathrm{E}$ scintillator, of course, has a finite thickness which is taken into account as follows. The range of particle can be written, to a very good approximation in the region of interest, as:

$$
R(E)=C Z^{2} M^{2-\gamma} E^{Y} \quad(C=\text { constant })
$$

The CsI crystals used in this experiment have a $\gamma$ of 1.7 . After a particle passes through the $\Delta \mathrm{E}$ scintillator its energy is $\mathrm{E}-\Delta \mathrm{E}$ and its range becomes

$$
R(E-\Delta E)=C Z^{3} M^{2-Y}(E-\Delta E)^{Y}
$$

It follows that

or

$$
\begin{aligned}
& R(E)=\Delta x+R(E-\Delta E) \\
& C Z^{2} M^{2}-V^{\gamma}=\Delta x+C Z^{2} M^{2-\gamma}(E-\Delta E)^{\gamma} .
\end{aligned}
$$

Solving for $\Delta E$, gives 


$$
\Delta E=\left[\frac{\Delta x}{C Z^{2} M^{1-Y}}+(E-\Delta E)^{Y}\right] 1 / Y .
$$

This function is plotted for several different particles in Figure 4. The dashed lines represent characteristic energy losses for particles which have penetrated the thick crysta 1 . One can readily see that a unique $\Delta E$ vs. E - $\Delta \mathrm{E}$ line exists for each stopping particle and this permits reliable particle identification. In fact, the separation of the lines is sufficiently large that resolution of the hydrogen and helium isotopes can be obtained by conventional scintillator techniques.

B. The Design of the Detector.

An internal view of the detector is shown in Figure 5. Both the $\Delta \mathrm{E}$ and $E-\Delta E$ scintillators are CsI(T1) crystals chosen for their high density, relatively linear light output, non-hygroscopic nature, and good mechanical properties. The thickness of the upper scintillator $\left(.45 \mathrm{gm} / \mathrm{cm}^{2}\right)$ was so chosen that an easily measurable light output and acceptable resolution would be attained for the minimum value of $\Delta \mathrm{E}$. A particle whose range equals the sum of the thicknesses of the $\Delta E$ scintillator and the light baffle separating the two crystals (see Figure 5 ) will have the minimum measurable energy. For protons this is approximately $15 \mathrm{MeV}$. The maximum letectable energy is determined by the thickness of the E - $\Delta E$ scinti11ator $\left(9 \mathrm{gm} / \mathrm{cm}^{2}\right)$. For protons, this is $79.3 \mathrm{MeV}$. Thus we have a detector capable of measuring protons in roughly the 15-80 MeV interval. Similarly the detector is sensitive to electrons in the 3-12 $\mathrm{MeV}$ interval, deuterons in the 20-106 MeV interval, tritons in the 24-126 MeV interval, and alphas in the 60-320 MeV interval.

The CsI scintillators are each $5 \mathrm{~cm}$ in diameter and separated by a distance of $10 \mathrm{~cm}$. This results in a geometry factor of $3.3 \mathrm{~cm}^{2}$-steradian 
at $15 \mathrm{MeV} /$ nucleon and $2.2 \mathrm{~cm}^{2}$-steradian at $80 \mathrm{MeV} /$ nucleon. The maximum angle of acceptance, 26 degrees, results in an average path length variation in the detector of approximately $3 \%$.

Each of the scintillators is viewed by an RCA 7151 photomultiplier. The $\Delta \mathrm{E}$ and $\mathrm{E}-\Delta \mathrm{E}$ phototubes are removed from the path of a particle entering the detector in order to minimize the amount of material traversed by the particle. It was found that a coating of highly reflective white paint $\left(\mathrm{TiO}_{2}\right.$ base) on the inner walls of the detector housing gave the best resolution. An effective integration cavity for the light from the scintillator is thus formed.

C. The Flight Circuitry.

The flight circuitry has undergone a gradual evolution starting in 1961 with the earliest version, termed the "Mark I", and ending with the "Mark VII" in 1965. The Mark I and Mark II versions are not significantly different and will be discussed here as one single configuration. The Mark IV through Mark VII versions are also quite similar and will be discussed in the next chapter in connection with the modified detector.

A block diagram for the Mark I and II circuitry packages is shown in Figure 6. The outputs of each of the RCA 7151 phototubes viewing the $A(\Delta E)$ and $B(E-\Delta E)$ scintillators are amplified, shaped, and sent into each of two 128 channel pulse height analyzers. These analyzers typically have integral linearities better than $1 \%$. It is required that an $A B$ coincidence occur before the pulse height analysis can take place. The 14 bits of information from the two pulse height analyzers are then recorded in 16 track magnetic tape. The output of the $C$ plastic anti-coincidence shield (Figure 3) is sent into a discriminator with a threshold corresponding to a few tenths of an MeV of energy lost in the $\mathrm{C}$ scintillator. The 
discriminator output is then also recorded on the magnetic tape. Those events having a non-zero recording from the plastic anti-coincidence scintillator can be identified in the subsequent data analysis. Thus, we can limit our analysis only to events of the type $A B \bar{C}$.

In order to increase the dynamic range of the circuitry a gain switching procedure was incorporated. In alternate time periods the gain was changed between two values differing roughly by a factor of 5 . The high gain mode was adjusted to optimize the position of the proton and electron distributions, and the low gain mode was adjusted to optimize the position of the alpha particle line. The occurrence of a gain change was recorded by simulating an event falling in the $64^{\text {th }}$ channel of the A and B pulse height analyzers. The probability of a real event falling in this particular pair of channel numbers is quite small. Also, the gain change markers occur at regularly spaced time intervals throughout the flight. Thus, the probability of confusing a real event with a gain change marker is minimized.

The magnetic tape was run at a constant, well-regulated speed of 10 inches per minute throughout the entireduration of the flight. The size of the tape reel permitted data accumulation for a period of about 18 hours, providing a comfortable margin in excess of the average fiight duration of 10 to 15 hours. Time resolution was obtained by simply measuring the distance along the tape to where the event occurred. In addition the gain change marker was used as a consistency check. Gain switching occurs at known and constant time intervals throughout the flight and thus provides independent timing information.

D. The Modified Detector.

In 1965, the last year of the balloon flight series reported here, a 
modified version of the $d E / d x$ and $E$ detector was flown for the first time. The physical construction of the detector itself remained essentially unchanged except for the addition of a third CsI(T1) scintillator directly behind the thick E - $\Delta E$ scintillator (B). The scintillator configuration for the modified detector is shown in Figure 7. The added CsI scintillator and the altered plastic anti-coincidence shield are both viewed by the same phototube. A phoswitch is used to distinguish between the slow light pulses from the CsI and the fast pulses from the plastic. A third pulse height analyzer has also been added to measure the light output from the additional CsI scintillator.

As with the original version of the detector, a11 events producing an $A B$ coincidence are recorded. The various possible types of acceptable events are labelled (1), (2), and (3) in Figure 7. Trajectory (1) is characterized by an output in the zeroth channel of the $C$ pulse height analyzer. This is just the $\mathrm{dE} / \mathrm{dx}$ and $\mathrm{E}$ or stopping particle mode of operation of the detector. Trajectory (2) will have a non-zero recording in the $C$ pulse height analyzer but will have a zero output from the phoswitch; this corresponds to the penetrating particle mode of operation. Trajectory (3) has a non-zero output from the phoswitch and is rejected in the subsequent data analysis.

To understand the response of the detector to penetrating particles, refer back to Figure 4. It will be recalled that the solid lines represented the energy losses respectively in the $A$ and $B$ crystals under the restriction that the particle stopped in the B crystal. If we now relax this restriction and allow the particle to penetrate the thick crystal we obtain the dashed curves shown in Figure 4. Since the energy loss rate $\mathrm{dE} / \mathrm{dx}$ rapidly increases with decreasing energy, a particle stopping in the thick 
crystal will lose most of its energy near the end of its trajectory. If the particle is allowed to penetrate the thick crystal this high $\mathrm{dE} / \mathrm{dx}$ region no longer contributes to the energy loss in the thick crystal. Consequently the energy deposited in the thick scintillator abruptly begins to decrease rapidly. This then causes the sharp reversal of the curves shown in Figure 4.

The curves for both downward and upward moving penetrating particles are reproduced in Figure 8 . The solid lines in this figure are identical to the dashed lines in Figure 4. The label $\mathrm{E}-\Delta \mathrm{E}$ has been retained on the horizontal axis for simplicity. It must be remembered however, that the particle is no longer stopping in the thick crystal. The alpha particle incident energy in MeV/nucleon is denoted by the numbers along the alpha-particle line. One sees that the curves for upward and downward moving particles converge as the incident energy increases and eventually become identical at energies corresponding to minimum ionization. Of course, the lines shown in Figure 8 are idealizations. In reality the values of $\Delta E$ and $E-\Delta E$ will be scattered about these lines due to statistical fluctuations in energy loss and light output. Distribution widths range from as 1ittle as $3 \%$ FWHM for stopping particles to as much as $40 \%$ FWHM for energetic penetrating particles. This broadening has the effect of making the lines for upward and downward moving particles converge at energies considerably less than minimum ionization. In the presence of both downward and upward moving particles an upper limit is thus imposed on energies measurable by the detector.

The exact value of this upper limit will vary with the type of particle under consideration. Heavier particles will deposit more energy in the scintillators than light particles and consequently will be more 
easily resolved. One thus expects a higher upper 1 imit for heavy particles. An additional difficulty is presented by the fact that the alpha particle minimum ionization point lies very close to the proton line. Such an effect will also exist for adjacent pairs of lines for heavier particles. There will thus be an incident energy interval for each type of particle in which the pairs of 1 ines will not be separable.

These difficulties are partially solved by using a third available parameter, namely the output of the C CsI scintillator. Previously it was only possible to analyze the data in a two dimensional representation. With the third parameter provided by the C crystal a three dimensional analysis becomes possible. The background becomes distributed over a three dimensional space instead of being compressed into a plane and thus is more easily separable from true events. Similarly, upward and downward moving particles and adjacent pairs of lines are more easily distinguished.

It is, of course, difficult to construct a three dimensional display of the data. An alternative approach having a similar effect is to use the third parameter to impose consistency criteria on the data and then display it in a two-dimensional array. For a given type of particle the outputs of the $\mathrm{A}$ and $\mathrm{B}$ scintillators uniquely determine (neglecting statistical fluctuations) the output of the C scintillator. Thus for an event producing a given (A, B) coordinate pair, one can require that the $C$ coordinate be within certain suitably chosen limits about the theoretically predicted value. Very broad limits $( \pm 40 \%)$ were used to insure that no true events were rejected. However, since the background distribution is much broader than the distributions of the characteristic particle lines, a substantial reduction in background was achieved. 
A block diagram of the Mark VII circuitry package is shown in Figure 9. In this version three 512 channel pulse height analyzers are used. Sufficient dynamic range is thus available so that gain switching is not necessary. The pulse rise-time discriminator (phoswitch) has also been designed to operate over a wide dynamic range of pulse height. It is not a standard item and has several unique features worthy of mention here.

A schematic diagram of the pulse rise-time discriminator is shown in Figure 10. Positive pulses from the tenth dynode of the $\mathrm{C}$ phototube are sent into an isolating stage $(\mathrm{Q} 1, \mathrm{Q} 2)$ and then differentiated. The differentiated pulse is then passed through three stages of amplification with a total gain of 1000 . At each amplification stage, diode limiting is used to prevent pulse widening for very large input pulses. The output of the last amplification stage is then fed into a double pulse monostable $(Q 11, Q 12)$. This circuit generates a positive 0.3 volt, 100 nanosecond pulse at the leading edge of the differentiated input pulse and a negative 0.3 volt, 100 nanosecond pulse at the zero crossover point of the input pulse. The positive portion of the output of the double pulse monostable is then amplified $(Q 13, Q 14)$ and differentiated $(C 32, R 57)$. The negative portion of this differentiated pulse is used to drive a single pulse monostable $(Q 15, Q 16)$ which generates a standard pulse .75 microseconds wide. This pulse and the negative 100 nanosecond pulse from the double pulse monostable are fed into a diode AND gate (CR6,CR7). For a fast (plastic) input pulse the negative portion of the output of the double pulse monostable occurs less than $.75 \mu \mathrm{sec}$ after the standardized pulse generated by $\mathrm{Q} 15, \mathrm{Q} 16$. If the input pulse is slow (CsI) no coincidence will occur. The output of the AND gate is fed into another single pulse monostable whose output is then inverted. This output and the output 
from the $A B$ coincidence circuit are then fed into another AND gate (Q20,Q21). Thus an output from the pulse rise time discriminator is obtained only for an event of the type $A \overline{A B C}_{f}$, where the subscript $f$ denotes a fast pulse from the $\mathrm{C}$ phototube. This output is then recorded on magnetic tape along with the pulse height information for each event.

When a primary particle undergoes a nuclear interaction within the $B$ scintillator, it is possible that the reaction products might simultaneously deposit energy in both the plastic and CsI portions of the C scintillator. It is then possible that the slow component of the light from the CsI might overwhelm the fast component from the plastic and thereby produce a spurious $A \overline{B C}_{f}$ event. One can, however, discriminate against such events in the subsequent data analysis by imposing the consistency criteria discussed earlier.

The logic circuitry used in the Mark VII is essentially identical to that used in the earlier Mark I and II circuitry packages. As before, an $\mathrm{AB}$ coincidence is required to open the linear gates and initiate the pulse height analysis and recording sequence. The output of the three pulse height analyzers and the phoswitch are recorded on 16 track magnetic tape in the form of two sixteen bit words. As before, the constant tape speed is used to obtain time resolution. 


\section{THE BALLOON FLIGHT SERIES}

The data to be discussed here were obtained during a series of 15 successful balloon flights beginning in June, 1961 and ending in June, 1965. A total of 30 launches were made with a success rate of $50 \%$. Most of the failures were due to balloon bursts usually occuring near the tropopause where atmospheric temperatures are lowest. The balloons used are the largest currently available, ranging in volume from 3 to 10 million cubic feet when fully inflated. This huge surface area of extremely thin material (usually $\frac{1}{2}$ to $1 \mathrm{mil}$. polyethylene) represents a very fragile structure. During the ascent a balloon's volume commonly expands by a factor of 300. Stresses occuring during this expansion and unfolding are a likely cause of balloon failure.

Since our data were recorded on magnetic tape and no telemetry was used, it was essential to recover the balloon payload after cut-down. During a typical flight a ba11oon would travel a distance of approximately 500 miles. Frequently the impact area would be in extremely difficult terrain. It is a tribute to the tracking and recovery crews that all successfully launched payloads were recovered.

A summarization of the pertinent flight data is presented in Table $I$. The majority of the flights were launched from Fort Churchill, Manitoba where the geomagnetic cut-off is less than $20 \mathrm{MeV}$ for protons. Since the purpose of these flights was to monitor the primary cosmic ray intensity at low energies, a low geomagnetic cut-off was required. Several flights were made at lower geomagnetic latitudes with correspondingly higher geomagnetic cutoffs.

The geomagnetic cutoffs for the low latitude flights were al1 wel1 above the maximum energy acceptable by the detector. Thus the data obtained 
TABLE I

FLIGHT DATA

\begin{tabular}{|c|c|c|c|c|c|c|}
\hline $\begin{array}{l}\text { Flight } \\
\text { Number }\end{array}$ & $\begin{array}{l}\text { Geographic } \\
\text { Latitude }\end{array}$ & Location & Date & $\begin{array}{l}\text { Duration } \\
\text { at Altitude }\end{array}$ & $\begin{array}{l}\text { Avg. Floating } \\
\text { Altitude }\left(\mathrm{gm} / \mathrm{cm}^{2}\right)\end{array}$ & $\begin{array}{l}\text { Geomagnetic } \\
\text { Cutoff }(\mathrm{BeV})\end{array}$ \\
\hline $61-1$ & $59^{\circ}$ & $\begin{array}{l}\text { Ft. } \\
\text { Manitoba }\end{array}$ & $7 / 31 / 61$ & $8 \mathrm{hr} .20 \mathrm{~min}$. & 3.7 & $<.02$ \\
\hline $61-2$ & $43.6^{\circ}$ & $\begin{array}{l}\text { Sioux Fa11s, } \\
\text { S. D. }\end{array}$ & $12 / 05 / 61$ & $2 \mathrm{hr} .30 \mathrm{~min}$. & 3.9 & .99 \\
\hline $62-1$ & $59^{\circ}$ & Ft. Churchil1 & $6 / 18 / 62$ & $6 \mathrm{hr}$. & 3.9 & $\therefore .02$ \\
\hline $62-2$ & $59^{\circ}$ & $\begin{array}{c}\text { Ft. } \\
\text { Manitoba }\end{array}$ & $6 / 23 / 62$ & 12 hr. $10 \mathrm{~min}$. & 4.0 & $<.02$ \\
\hline $62-3$ & $59^{\circ}$ & $\begin{array}{c}\text { Ft. Churchill } \\
\text { Manitoba }\end{array}$ & $6 / 30 / 62$ & $9 \mathrm{hr}$. & 3.4 & $\therefore .02$ \\
\hline $62-4$ & $59^{\circ}$ & $\begin{array}{c}\text { Ft. Churchill } \\
\text { Manitoba }\end{array}$ & $7 / 15 / 62$ & $12 \mathrm{hr} .30 \mathrm{~min}$. & 3.4 & $\therefore .02$ \\
\hline $63-1$ & $59^{\circ}$ & $\begin{array}{c}\text { Ft. Churchill } \\
\text { Manitoba }\end{array}$ & $6 / 15 / 63$ & $10 \mathrm{hr} .50 \mathrm{~min}$ & 3.2 & $\therefore .02$ \\
\hline $63-2$ & $59^{\circ}$ & Ft. $\begin{array}{ll}\text { Churchil11 } \\
\text { Manitoba }\end{array}$ & $6 / 24 / 63$ & $7 \mathrm{hr} .30 \mathrm{~min}$. & 3.8 & $\therefore .02$ \\
\hline $64-1$ & $59^{\circ}$ & Ft. $\begin{array}{l}\text { Churchi11 } \\
\text { Manitoba }\end{array}$ & $6 / 18 / 64$ & $8 \mathrm{hr} .50 \mathrm{~min}$. & 3.0 & $<.02$ \\
\hline $64-2$ & $59^{\circ}$ & 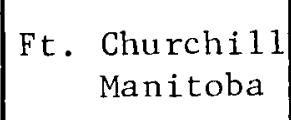 & $6 / 21 / 64$ & $9 \mathrm{hr} .10 \mathrm{~min}$ & 2.7 & $\therefore .02$ \\
\hline $64-3$ & $59^{\circ}$ & $\begin{array}{c}\text { Ft. Churchill } \\
\text { Manitoba }\end{array}$ & $6 / 24 / 64$ & $9 \mathrm{hr} .30 \mathrm{~min}$. & 3.2 & $\therefore .02$ \\
\hline $64-4$ & $44.3^{\circ}$ & $\begin{array}{l}\text { Fairibault, } \\
\text { Minn. }\end{array}$ & $8 / 06 / 64$ & $9 \mathrm{hr}$. & 5.0 & .79 \\
\hline $64-5$ & $32^{\circ}$ & $\begin{array}{c}\text { Palestine } \\
\text { Texas }\end{array}$ & $10 / 17 / 64$ & $10 \mathrm{hr} .30 \mathrm{~min}$. & 3.0 & 3.6 \\
\hline $65-1$ & $59^{\circ}$ & $\begin{array}{l}\text { Ft. Churchil1 } \\
\text { Manitoba }\end{array}$ & $6 / 12 / 65$ & $10 \mathrm{hr} .30 \mathrm{~min}$. & 2.8 & .02 \\
\hline $65-2$ & $59^{\circ}$ & $\begin{array}{l}\text { Ft. } \\
\text { Manitoba }\end{array}$ & $6 / 23 / 65$ & $8 \mathrm{hr}$. & 2.8 & .02 \\
\hline
\end{tabular}


during these flights consists entirely of atmospheric secondaries and re-entrant albedo. The purpose of these flights was, in fact, to obtain values for the atmospheric secondary flux so that corrections could be applied to the Churchill data.

One can see from Table I that floating altitudes ranging between 2.8 and $5.0 \mathrm{gm} / \mathrm{cm}^{2}$ of residual atmosphere were reached. The lower limit of $2.8 \mathrm{gm} / \mathrm{cm}^{2}$ is close to the optimum condition attainable with the presently available balloon technology. A typical altitude curve is shown in Figure 11. One can see that the floating altitude remained constant within a few tenths of a $\mathrm{gm} / \mathrm{cm}^{2}$.

The smoothed sunspot number and Mt. Washington neutron monitor rate (on an inverted scale) are shown in Figure 12. The date of each group of flights is denoted by an arrow. The letter and number denote respectively the location and number of successful flights. One can see that the balloon flight series covered approximately half of the time interval between solar maximum and solar minimum. During this period the Mt. Washington neutron monitor intensity varied between approximate1y 2200 and 2500 . This is approximately $3 / 5$ of the total neutron monitor variation observed between solar maximum and solar minimum.

Three point proton energy spectra were obtained from each of the flights at energies of 29, 48 and $69 \mathrm{MeV}$.at the detector. As stated earlier the energy "window" seen by the detector is $20-80 \mathrm{MeV} / \mathrm{nucleon}$. The balloon flight altitudes were characteristically $3-4 \mathrm{gm} / \mathrm{cm}^{2}$ of residual atmosphere. The $20-80 \mathrm{MeV} /$ nucleon interval when transformed to the top of the atmosphere then typical1y becomes 70-100 MeV/nucleon. Since the energy interval at the top of the atmosphere is so narrow we have 
combined the three points measured at the detector into a single point at the top of the atmosphere. The data then consist primarily of a single point on the primary differential energy spectrum with an energy of approximately $85 \mathrm{MeV} /$ nucleon measured as a function of time from 1961 to 1965 .

We have yet to discuss the largest correction to the proton data, namely the atmospheric secondaries produced above the detector. This will be done in detail in Chapter VI. The procedure adopted consisted of first subtracting the secondary intensity at the altitude of the detector from the total intensity observed at the detector. We thus obtained the primary intensity at the detector. This intensity must then be multiplied by the ratio of the width of the energy interval at the detector to that at the top of the atmosphere (typically a factor of 1.5) to obtain the intensity at the top of the atmosphere. 


\section{DATA ANALYSIS}

\section{A. Stopping Particle Analysis}

As was mentioned previously, the raw data from each flight are in the form of binary bits recorded on a 16 track magnetic tape. A buffer system is used to convert the flight tape into one which is compatible with the IBM 7094 computer. A variety of computer programs can then be used to read this tape.

The most useful and revealing format for data presentation is the computer generated plot shown in Figure 13. This is effectively a plot of $\Delta \mathrm{E}$ vs. $\mathrm{E}-\Delta \mathrm{E}$. The vertical scale is simply the direct unnormalized output of the $A(\Delta E)$ pulse height analyzer. Similarly the horizontal scale represents the output of the $B(E-\Delta E)$ pulse height analyzer. At each pair of coordinates on the plot the total number of events during a given time period producing that particular combination outputs of the $A$ and $B$ pulse height analyzers is printed. The plot represents a distribution function $\varphi(\Delta E, E-\Delta E)$ giving the number of events as a function of $\triangle E$ and $E-\Delta E$. Figure 13 contains the first 128 channels of pulse height analyzer data from flight 65-1. One can readily distinguish a proton line. The end point of this line is well-defined. This corresponds to a proton whose incident range equals the sum of the thicknesses of the $A(\Delta E)$ and the $B(E-\Delta E)$ scintillators plus a very small contribution from the light shield between the scintillators. The proton endpoint thus provides an in-flight energy calibration of the detector.

One can also without too much difficulty resolve a deuteron line lying above the proton line. A simple visual inspection is insufficient to determine whether or not a triton line exists, however, detailed evidence for the presence of tritium will be presented later. 
A similar plot containing a11 512 channels of pulse height analyzer data is shown in Figure 14. The horizontal and verticle scales have both been compressed by a factor of four relative to Figure 13 . One can readily resolve an alpha particle line. For particles having the same velocity the alpha particle energy loss is a factor of four higher than the proton energy loss. Since the scale in Figure 14 has been compressed by a factor of four the alpha particle line is essentially identical to the proton line in Figure 13.

In the older versions of the circuitry where only 128 channels were available this compression was accomplished by a gain change. This, of course, led to a smaller data accumulation time for each type of particle and consequently poorer statistics. The improved circuitry used in 1965 permitted simultaneous analysis of both proton and alpha particle fluxes during the entire flight.

In Figures 13 and 14 it is apparent that there is a certain amount of background distributed over the array. The large accumulation of events in the lower left corner of Figure 13 is due to low energy (2-12 $\mathrm{MeV}$ ) electrons stopping in the detector. Part of the background in the vicinity of the proton line is associated with the tail end of this electron distribution. The remainder of the background seen in the array is attributed to nuclear interactions occurring within the detector. For example, a particle might enter through the side of the detector and produce a star in the thick scintillator. It is then possible that one of the reaction products might escape throught the upper surface of the thick scintillator and enter the thin scintillator. Such a spurious event could produce an $\mathrm{AB} \bar{C}$ coincidence and be analyzed. 
During a typical balloon flight the penetrating particle rate is approximately five times as large as the stopping particle rate. This is indicative of the significant probability of a nuclear interaction occurring within the detector.

An empirical approach has been adopted to correct for this type of background produced within the detector. Figure 15 illustrates the background correction procedure. Three mass histograms are plotted corresponding to different energy intervals. The histograms were obtained by constructing a series of lines parallel and equidistant from the proton line and summing the total number of events between each adjacent pair of lines. The horizontal scale in Figure 15 is then just the line number or perpendicular distance from the proton line in arbitrary units.

A clearly resolved proton line is seen in all three energy intervals. A distinct valley between the proton and deuteron distributions is observed. The dashed lines in each of the histograms represent an extension of the background distribution through the region of the proton line. An upper limit of approximately $5 \%$ of the total number of protons can be placed on this type of background correction. We will see later that other much larger corrections due to atmospheric secondaries must be made.

To obtain energy spectra at the detector it is necessary to know the incident energy as a function of light output in either the thin or the thick crystal. The energy loss in CsI as a function of incident energy is well known. One of the most complete compilations of range and energy loss rate as a function of energy is found in the tables of Barkas and Berger [1964]. These have been used extensively through- 
out the entire data analysis.

The light output of CsI as a function of energy loss (dL/dE vs. $\mathrm{dE} / \mathrm{dx}$ ) is not as wel1 known. A summary of al1 the existing experimental data is presented in a paper by Gwin and Murray [1962]. Unfortunately a gap in the $d L / d E$ vs. $d E / d x$ data exists in the region of interest for this detector. From an interpolation it is possible that a non-1inearity of as much as $20 \%$ could exist in the light output of CsI in this region.

Two items of evidence indicate that the non-1inearity in light output is small ( $\pm 5 \%)$. First, the theoretically predicted proton line (assuming no non-linearities) is in reasonably good agreement with the empirical 1ine. Second, accelerator calibrations of the detector have been carried out at the Oak Ridge Isochronous Cyclotron (ORIC) and the Harvard cyclotron. These calibrations consisted of exposing the detector to monoenergetic proton, deuteron, and alpha particle beams of varying but known energies. Unfortunately, they were not entirely successful, and the information obtained on light output non-linearities was incomplete. However, the existing data agrees with the original hypothesis that the non-linearities are small.

The accelerator calibrations also provided information on the resolution of the detector at various energies. For stopping protons the resolution in the thin scintillator was typically $20 \%$ (full width at half maximum). The resolution in the thick scintillator at the same energies was characteristically $3-5 \%$, except at very low energies where it became worse. It is, of course, expected that the resolution in the thick scintillator will be better since, first, photon statistics are better and, second, the particles are required to stop in the 
scintillator. For this reason the energy loss in the thick crystal was used to determine a particle's energy.

Atmospheric secondaries constitute the largest source of background in our experiment. These particles are produced by collisions of primary cosmic rays with air nuclei. Copious quantities of low energy protons $(\lesssim 30 \mathrm{MeV})$ are thus produced due to nuclear evaporation. Owing to their low energies, however, these particles do" not travel very far in the atmosphere. The multiplicity of higher energy reaction products is smaller [see, for example, Powe11, Fowler, and Perkins, 1959], but because of their greater range, they survive longer and thus can become a significant source of background. Since the energy window of our detector is $20-80 \mathrm{MeV}$ for protons, it will be these higher energy secondaries which make the largest contribution to the background.

In 1965 the secondary intensity at $3 \mathrm{gm} / \mathrm{cm}^{2}$ was $50 \%$ of the total proton intensity at the detector in the 20-80 $\mathrm{MeV} / \mathrm{nucleon}$ interval. In 1961 the secondary contribution was even larger, $80 \%$ of the total proton intensity. This large correction is then the greatest single contribution to the total experimental uncertainty. We are confronted with a situation where we must subtract two intensities which differ only by 20 to $50 \%$. The fractional error in the difference then becomes much larger than the fractional error in either of the original intensities. Corrections to the stopping alpha particle intensities are somewhat more straightforward. The small number of events (typically 30-50 during a single flight) does not permit the construction of mass histograms as was done for the proton distributions. It is expected, however, that the alpha particle background produced within the detector will be even smaller than the proton background since the tail of the 
electron distribution will not contribute. The ionization loss correction is the same as that for protons when done on an energy/nucleon basis.

A correction must be made for nuclear interactions in the atmosphere above the detector. Two effects must be considered. First, a certain fraction of the primary alpha particles will undergo catastrophic interactions with atoms in the atomsphere. This will cause a degradation in the alpha particle intensity observed at the detector. Second, heavier particles will undergo fragmentation in the atmosphere and a certain fraction of these interactions will produce secondary alpha particles. This will contribute a small increase in the alpha particle intensity at the detector. A number of experimentors [McDonald, 1956; Davis et al.; 1956; Webber and Ormes, 1963] have measured the alpha particle intensity as a function of depth in the atmosphere and have arrived at an "effective" absorption mean free path for alpha particles in air. The adjective "effective" is used since the mean free path results from a combination of the two effects discussed above. We have used the value of $52 \mathrm{gm} / \mathrm{cm}^{2}$ obtained by Webber and Ormes for correcting the alpha particle intensity.

One must also consider the effect of alpha particles undergoing nuclear interactions within the detector itself. The thickness of the B scintillator $\left(9 \mathrm{gm} / \mathrm{cm}^{2}\right)$ is approximately one tenth of an interaction mean free path for alpha particles in CsI. Thus one expects about $5 \%$ of the incident alpha particles to interact in the detector, since on the average, the path length of a stopping particle will be approximately half the total thickness of the crystal. If an elastic collision occurs the residual range of the alpha particle will be unchanged and the alpha particle will very probably lose all of its remaining energy in the $B$ 
scintillator. If an inelastic collision occurs it is unlikely that any of the charged reaction products will have ranges larger than the residual range of the alpha particle. It is possible, however, that some energy might be carried off by secondary neutrons, since they would lose no energy due to ionization. Events of this type, however, will be quite rare, and consequently their effects will be neglected in the data analysis.

B. Penetrating Particle Analysis

As with stopping particles, the most convenient method of displaying the data is a computer plot similar to those in Figures 13 and 14. We are now interested in particles which traverse the CsI portion of the $\mathrm{C}$ scintillator, but produce no output in the plastic guard scintillator. Consequently we will look at events of the type $A B \bar{C}_{p}$ where the subscript $p$ refers to the plastic portion of the $C$ scintillator. Such a plot is shown in Figure 16 using the data from flight 65-1. This is a 128 by 128 channel plot which is only $1 / 16$ of the total array (since 512-channel pulse height analyzers were used in 1965). The dominant feature is the clustering of events about a line which gradually rises as one moves along the horizontal axis. This is the high energy portion of the penetrating alpha particle line. To understand this more fully we refer back to Figure 4. The empirical line in Figure 16 then corresponds to a segment of the dashed portion of the alpha particle line in Figure 4. This portion of the array includes alpha particles with energies greater than $200 \mathrm{MeV} /$ nucleon. Referring to Figure 8 , we see the location of the penetrating proton line relative to the penetrating alpha particle line. The proton line in Figure 16 is thus completely obscurred by the high density of events 
in the lower portion of the plot. Analysis of the proton line was made extremely difficult by an instrumental effect (to be discussed 1ater) which caused high background in the region of the proton 1ine, and also by the poor resolution at high energies due to statistical fluctuation in light output and energy loss. For this reason only alpha particle intensities were analyzed in the penetrating particle mode of operation of the detector.

As has been mentioned, a third parameter is available to aid in identifying a particle's charge, mass, and energy: the energy loss in the C cesium iodide scintillator. A computer program was written which compared the energy losses respectively in the $\mathrm{B}$ and $\mathrm{C}$ scintillators. For a particular type of particle the energy loss in B uniquely determines the energy loss in $\mathrm{C}$ (neglecting statistical fluctuations in energy loss and light output). The program demanded that the $\mathrm{B}$ and $\mathrm{C}$ outputs bear the proper relation to each other (allowing for a $\pm 40 \%$ fluctuation). Those events which did not satisfy this condition were discarded. Figure 17 is a plot of only those events which satisfy the above criteria. Comparing Figure 17 with Figure 16, one can see that the background surrounding the alpha particle line has been significantly reduced.

In Figure 18 we have a plot of the entire penetrating alpha particle line. As with Figure 17 consistency criteria have been imposed. Several features of this plot are worth mentioning. First, we note that the density of points along the alpha particle line decreases rapidly as one goes towards lower energies. The energy loss rate is a rapidly increasing function of energy as one goes toward lower energies. Consequently, for a given increment in energy loss (or increment along 
the alpha particle line) the increment in incident energy becomes increasingly larger. Thus, if the incident energy spectrum is not rapidly changing, one expects the density of points along the alpha particle line to be much less at lower energies. Second, a clustering of events is noted near the low energy end and above the alpha particle line. These points fall near the expected position of the lithium line. The poor statistics do not permit a meaningful analysis of these events. However, they do provide an indication of the usefulness for heavier particles of a satellite version of the detector where data accumulation can take place over a much longer period of time. Third, there is a clustering of points near and parallel to the vertical axis of the plot. This is the instrumental effect mentioned earlier. Unfortunately, there was no overflow indicator in the pulse height analyzers. The output of the linear portion of the circuitry is limited at approximately six volts. This would effectively correspond to channel 600 in the pulse height analyzer. Since the pulse height analyzer is modulo 512, such a six volt signal would fall in the 88th channel. This clustering is in fact concentrated in channels 0 through 90 along the horizontal axis.

Cross-sections of the penetrating alpha particle line for various energy interva1s are shown in Figure 19. The corrections for background produced within the detector are somewhat larger than they were in the stopping particle mode. The background is seen to be particularly large in the 270-330 MeV/nucleon interval. This is due to the overflow discussed earlier.

One cannot neglect nuclear interactions of penetrating alpha particles within the detector. We shall assume that all peretrating 
particles that interact in the detector are rejected. The rejection occurs in one of two ways. First, it is possible that a reaction product or scattered alpha particle will enter the plastic guard scintillator. More important, we have required consistency between the energy losses in the $\mathrm{B}$ and $\mathrm{C}$ scintillators. It is unlikely that a penetrating particle undergoing an interaction within the detector will satisfy these requirements. We must then correct for the degradation in intensity due to such interactions; this correction is of the order of $10 \%$. 


\section{TIME VARIATIONS IN THE LOW ENERGY \\ PROTON AND ALPHA PARTICLE INTENSITIES}

\section{A. General Remarks}

We shall be concerned here only with the long-term temporal variations in the proton and alpha particle intensities. With a single balloon flight typically lasting 10 hours, it is difficult to obtain meaningful information on short-term fluctuations in the cosmic ray intensity lasting typically several days. The long-term time variation in the cosmic ray intensity has a period of some eleven years. Thus our balloon flight measurements, lasting typically ten hours, may be considered as a set of discrete points of the cosmic ray intensity as a function of time during the period from 1961 to 1965 . These points correspond to the cosmic ray intensities at an energy of approximately $85 \mathrm{MeV} /$ nucleon.

A summary of the proton and alpha particle measurements obtained in this experiment is presented in Table II. Included here are only flights which took place at Fort Churchill, Manitoba since the lower latitude flights yield no information on the primary intensity at low energies. The table contains both the total uncorrected proton and alpha particle intensities at the detector in addition to the corrected primary intensities at the top of the atmosphere. One can see from the table that in 1961 (Flight 61-1) the secondary contribution was largest, approximately $80 \%$ of the total intensity at the detector.

Also included in Table II are the average energies at the top of the atmosphere for each $f 1$ ight (the same in MeV/nucleon for both protons and alpha particles). The small variations in these values are due to the variation in floating altitude from one balloon flight to the next. 
TABLE II

SUMMARY OF INTENSITIES AT FORT CHURCHILL

\begin{tabular}{|c|c|c|c|c|c|}
\hline \multirow[b]{2}{*}{$\begin{array}{l}\text { Flight } \\
\text { Number }\end{array}$} & \multicolumn{2}{|c|}{ Protons } & \multicolumn{2}{|l|}{ Helium } & \multirow[b]{2}{*}{$\begin{array}{l}\text { Average energy } \\
\text { at top of } \\
\text { atmosphere } \\
\text { (MeV/nucleon) }\end{array}$} \\
\hline & \begin{tabular}{|l|} 
Total \\
Intensity \\
at \\
Detector \\
\end{tabular} & $\begin{array}{l}\text { Primary } \\
\text { Intensity } \\
\text { at top of } \\
\text { Atmosphere } \\
\end{array}$ & $\begin{array}{l}\text { Total Intensity } \\
\text { at Detector }\end{array}$ & $\begin{array}{l}\text { Primary } \\
\text { Intensity } \\
\text { at top of } \\
\text { Atmosphere }\end{array}$ & \\
\hline $61-1$ & $.88 \pm .05$ & $.24 \pm .12$ & $.07 \pm .02$ & $.12 \pm .04$ & 89.1 \\
\hline $62-1$ & $.85 \pm .09$ & $.26 \pm .20$ & --- & -- & 89.6 \\
\hline $62-2$ & $.94 \pm .04$ & $.33 \pm .13$ & -- & -- & 89.8 \\
\hline $62-3$ & $.86 \pm .06$ & $.26 \pm .14$ & --- & -- & 85.4 \\
\hline $62-4$ & $.92 \pm .04$ & $.35 \pm .12$ & --- & --- & 85.4 \\
\hline $\begin{array}{l}1962 \\
(\operatorname{avg} .)\end{array}$ & $.90 \pm .02$ & $.32 \pm .09$ & $.09 \pm .02$ & $.15 \pm .03$ & 87.6 \\
\hline $63-1$ & $1.06 \pm .07$ & $.59 \pm .15$ & --- & --- & 83.8 \\
\hline $63-2$ & $1.08 \pm .07$ & $.39 \pm .17$ & -- & -- & 96.7 \\
\hline $64-1$ & $.93 \pm .09$ & $.70 \pm .11$ & -- & -- & 82.3 \\
\hline $64-2$ & $.99 \pm .08$ & $.74 \pm .10$ & -- & -- & 79.9 \\
\hline $64-3$ & $.99 \pm .98$ & $.73 \pm .10$ & --- & -- & 80.7 \\
\hline $\begin{array}{l}1964 \\
(\operatorname{avg} .)\end{array}$ & $.98 \pm .05$ & $.73 \pm .07$ & -- & $\ldots$ & 81.0 \\
\hline $65-1$ & $1.14 \pm .05$ & - & $.12 \pm .02$ & $.18 \pm .03$ & 80.7 \\
\hline $65-2$ & $1.15 \pm .05$ & - & $.17 \pm .03$ & $.26 \pm .05$ & 80.7 \\
\hline $\begin{array}{l}1965 \\
\text { (avg.) }\end{array}$ & $\mid 1.14 \pm .03$ & $.84 \pm .08$ & $.15 \pm .02$ & $.22 \pm .03$ & 80.7 \\
\hline
\end{tabular}

NOTE: Al1 intensities are in particles $/ M^{2}-M e V-s e c-s t e r$. 
The average kinetic energy at the top of the atmosphere for all balloon flights at Fort Churchill is $85 \mathrm{MeV} /$ nucleon. The maximum individual deviations from this average are $\pm 5 \mathrm{MeV} /$ nucleon. A small error will be introduced in our intensity vs. time curve due to the variation in the average energy at each point. However, the expected variation in the energy spectrum over this small interval ( $85 \pm 5 \mathrm{MeV})$ is insignificant compared to the errors introduced by the secondary proton correction. This error can therefore be safely neglected. Each measurement will consequently be considered as a single point on the differential energy spectrum at $85 \mathrm{MeV} /$ nucleon taken at a single instant in time.

The primary proton intensities listed for the years 1964 and 1965 are the satellite measurements of McDonald and Ludwig [1964」 and Balasubrahamanyan, et a1. [1966]. These are the only years for which satellite measurements are available at low energies. The simultaneous satellite and balloon observations of the cosmic ray intensity in 1964 and 1965 have been used to obtain the atmospheric secondary intensity. The corrected primary intersities obtained from the balloon measurements in 1964 and 1965 are then necessarily identical to the satellite-measured intensities during these years.

As has been stated, we are interested here in the quiescent or galactic cosmic ray intensity. We must, therefore, take note of two effects which could possibly lead to deviations from the quiet-time value of the cosmic ray intensity. The first of these, the Forbush decrease, manifests itself as a depression ( $\lesssim 10 \%$ ) in the sea-level neutron monitor rate. Second, it is possible that the quiescent galactic intensity could at times be contaminated by particles of solar origin, 
i.e. during a solar proton event. Sea level neutron monitors are sensitive only to the largest flare-associated solar proton events. We therefore need an alternate more sensitive indication of the presence of solar protons. This is provided by satellite-borne detectors which, on the average, measure particles of much lower energy than do sea level neutron monitors and balloon-borne detectors. Fortunately, the cosmic ray intensity was monitored by satellite-borne detectors during most of the time period covered by the balloon flight series.

Effects of the type mentioned above occurred in two of the five years encompassed by the balloon flight series. Flight $61-1$ on $30 \mathrm{July}$ 1961 took place during the last part of an active period beginning in mid-July. The activity was associated with an active region (Mt. Wilson 15353) which passed the central meridian on $14 \mathrm{July.} \mathrm{Several} \mathrm{flares} \mathrm{and}$ Forbush decreases were associated with this active region. A low energy solar proton event was observed on 26-27 July by Maehlum and O'Brien [1962] using detectors aboard the Injun I satellite. Associated with this was a Forbush decrease starting on 27 July. Evidence exists, however, that this activity had little or no influence on the intensities measured on 31 July during Flight 61-1. During this same period five balloon flights were made by Meyer and Vogt [1963] on $22 \mathrm{July}, 29 \mathrm{Ju} 1 \mathrm{y}$, 1 August, 6 August, and 8 August. Their detector, described earlier, was sensitive to cosmic ray energies at the top of the atmosphere as low as $80 \mathrm{MeV} /$ nucleon. During the last three flights of Meyer and Vogt no change in intensity was observed. A small enhancement at low energies was observed on 29 July attributed to the residual effects of the low energy solar proton event on $26-27$ July. Any possible solar proton contamination of our data taken on $30 \mathrm{July}$ must certainly be less than 
that observed by Meyer and Vogt on $29 \mathrm{July}$. Also, conditions had returned to normal by no later than 1 August, the date of Meyer and Vogt's next flight. We therefore submit that it is improbable that any significant deviation from the quiescent intensity occurred during Flight 61-1. One additional item of evidence exists to support this argument. On $7 \mathrm{July}$ 1961, wel1 before the onset of the period of activity, the low energy cosmic ray intensity was measured by Fichtel and co-workers [1964] using an emulsion stack. The low energy proton intensity obtained by them is in good agreement with that measured in Flight 61-1.

A very small solar proton event was observed on the Explorer XIV satellite on 15 June 1963, coincidentally with Flight 63-1 [Bryant, et a1.,1965]. The event started at $0300 \mathrm{UT}$ on 14 June and reached a maximum at 0300 UT on 15 June. During the balloon flight (1200-2200 UT) the solar proton event was therefore in its declining phase. A decrease in intensity was, in fact, observed during Flight 63-1. The time variation of the uncorrected energy spectrum observed at the detector during Flight 63-1 is shown in Figure 20. The flight was simply divided into three time intervals of equal length. The change in intensity is seen to be concentrated in the lowest energy point. The spectrum in the early portion of the flight is decreasing towards higher energies, whereas later in the flight it becomes flat or slightly increasing towards higher energies. A power law spectrum of the form $j \propto \mathrm{E}^{-\gamma}(\gamma=3-5)$ is typically observed during a solar proton event. This is qualitatively consistent with the spectral shape seen during the early portion of Flight 63-1. The slowly rising spectrum during the latter portion of the flight is consistent with the type of spectrums seen on other flights and expected during quiet conditions. We therefore, argue on the basis of 
the above remarks on the spectral shape that the solar proton contribution during the latter portion of Flight 63-1 cannot be large.

Additional support of this argument is provided by the results from Flight 63-2, launched on 24 June 1963. A decrease in intensity was also observed during the course of this flight. The Explorer XIV data of Bryant, et al., [1965] shows no activity on 24 June. However, on 25 June a small recurrent event was observed. It is likely that the depression in intensity during Flight 63-2 was due to disturbed magnetic fields associated with the stream of solar protons observed on the following day. The agreement of the intensities measured during the latter portion of Flight 63-1 with those measured early in Flight 63-2 suggests that these measurements are, indeed, representative of the quiescent galactic cosmic ray intensity.

No alpha particle data were obtained in 1963 and 1964. Since in 1963 we were able to use on ly a small portion of the data, it was impossible to obtain any statistically significant alpha particle information. In 1964 a circuitry modification unexpectedly caused a low-leve1 saturation of the photomultipliers in a region such that protons could be analyzed, but alpha particle information was lost.

B. The Cosmic Ray Intensity at $85 \mathrm{MeV} /$ nucleon from 1961 to 1965

The proton and alpha particle intensities obtained in this experiment are plotted as a function of time in Figure 21. Also plotted are measurements of other observers at similar energies. In some cases a small interpolation or extrapolation was involved due to small differences in average energy. Both the proton and alpha particle intensities appear to have increased by roughly a factor of four from 1961 to 1965 . This is to be compared with an increase of approximately $15 \%$ of the Mt. Washington neutron monitor rate during the same period of time. Since the 
Washington neutron monitor is sensitive on the average to primary particles with energies of approximately $3 \mathrm{BeV}$, we may conclude that the solar modulation of $85 \mathrm{MeV}$ protons was approximately 30 times larger than that for $3 \mathrm{BeV}$ protons.

The proton to alpha ratio at $85 \mathrm{MeV} /$ nucleon is plotted as a function of time in Figure 22. We have also included proton-alpha ratio measurement of McDonald and Webber [1964] made near solar maximum in 1959. This measurement is not directly comparable to the others since it was taken at a somewhat higher energy (200 MeV/nucleon). The protonalpha ratio in 1959, however, decreases as one goes toward lower energies. We may then consider the measurement of McDonald and Webber at $200 \mathrm{MeV} /$ nucleon as imposing an upper bound on the ratio at $85 \mathrm{MeV} / \mathrm{nucleon}$. This is indicated in Figure 22 by the arrow pointing downward from the 1959 point.

A number of different models for the solar modulation of galactic cosmic rays has been proposed. Elliot [1960] has developed a model based on an assumed dipole structure for the magnetic field. Direct satellite observations of the interplanetary magnetic field have shown, however, that it is not a simple dipole [see, for example, Ness, 1964 ]. Nagashima [1963] and Ehmert [1960] have assumed that there exists a potential difference between the earth's orbit and near galactic space and have developed a modulation model on this basis. Freier and Waddington [1965] have recently found good agreement between the experimental data and the predictions of this model. It is difficult to conceive how such a potential difference could be maintained in view of the high conductivity of the interplanetary plasma. However, the fact that the mathematical treatment appears to provide a good description of the experimental 
situation suggests that the solar modulation mechanism, whatever it may be, might simulate the effects of a conservative electric field.

We shall examine our data within the framework of the model for the solar modulation of galactic cosmic rays advanced by Parker [1963]. Parker has assumed that the degradation of the galactic cosmic ray intensity is caused by the solar magnetic field. Observations [see, for example, Ness, 1964] have shown that the solar magnetic field possesses a rather irregular structure superposed on an underlying Archimedes spiral configuration. The disorder is probably greater at large distances than in the immediate vicinity of the sun ( $<1$ A.U.). Parker treats these irregularities as scattering centers and uses a diffusion equation to describe the interaction of galactic cosmic rays with the solar magnetic field.

We shall consider several simple cases of Parker's model. The analysis will not include the possible effects of adiabatic deceleration, [Parker, 1965], since the present uncertainties in the data do not appear to permit such a detailed analysis. It will also be assumed that the diffusion coefficient is isotropic and depends only on the radial distance from the sun. Physically, this means that the solar magnetic field is taken to be spherically symmetric and that the disorder of the field is assumed to be so great that tendency toward non-random motion along the field lines can be neglected. Parker's diffusion equation can then be written as follows:

$$
\frac{\partial \mu}{\partial t}=-\vec{\nabla} \cdot(\vec{V} \mu)+1 / 3 w \lambda \nabla^{8} \mu,
$$


where

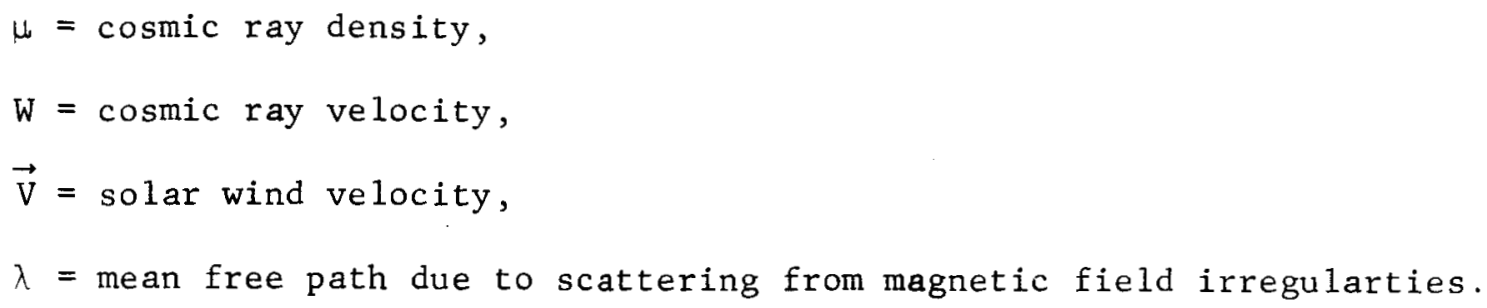

$$
-\frac{1}{r^{2}} \frac{\partial}{\partial r}\left(r^{2} V \mu\right)+1 / 3 w \lambda \frac{1}{r^{2}} \frac{\partial}{\partial r}\left(r^{2} \frac{\partial \mu}{\partial r}\right)=0
$$

Integrating equation (2) twice gives:

$$
\begin{aligned}
\mu(r, W) & =\mu(\infty, W) \exp \left[-\int_{r_{e}}^{\infty} \frac{3 V}{W \lambda} d r\right] \\
& =\mu(\infty, W) \exp \left[-\frac{3 V}{W} \int_{r_{e}}^{\infty} \frac{d r}{\lambda}\right]
\end{aligned}
$$

Experimentally, we have observed the differential intensity of protons and helium nuclei at the earth's orbit. We would like to relate this to the cosmic ray intensity outside of the sun's influence. To do this we rewrite equation (3) in the following form:

$$
\frac{d j}{d E_{n}}=\left(\frac{d j}{d E_{n}}\right)_{\infty} \exp \left[-\frac{3 V}{W} \int^{\infty} r_{e} \frac{d r}{\lambda}\right] \text {, }
$$

Where $\mathrm{E}_{\mathrm{n}}$ = energy/nucleon,

$$
\begin{aligned}
& \frac{\mathrm{d} j}{\mathrm{dE}_{\mathrm{n}}}=\text { differential intensity at earth's orbit, } \\
& \mathrm{r}_{\mathrm{e}}=\text { radius of earth's orbit. }
\end{aligned}
$$


Now we must examine the behavior of the exponent in equation (4). The quantities $V$ (the solar wind velocity) and $W=\beta c$ (the cosmic ray particle velocity) are known. But we do not know the value of the integral $\int_{r_{e}}^{\infty} \frac{d r}{\lambda}$. Parker has considered two simple cases. First, he has examined the case where the particle's radius of gyration, $a$, is much less than the scale size, $\ell$, of the magnetic irregularities. Then a scattering occurs each time a particle encounters a magnetic field irregularity, and we have simply $\int_{r_{e}}^{\infty} \frac{d r}{\lambda}=\nu$ where $\nu$ is the number of scattering centers between the earth's orbit and infinity. Second, Parker has considered the case where the radius of gyration is much larger than the scale size of the irregularities. Then the angular deflection is approximately $\Delta \theta \cong(\ell / a)$. A deflection of $90^{\circ}$ will therefore occur when a particle has made $(a / l)^{2}$ collisions. We can summarize these two simplified cases as follows:

$$
\begin{array}{rlrl}
\int_{r_{e} \frac{d r}{\lambda}}^{\infty} & =\nu & & \text { for } a<l \quad \text { (low energy) } \\
& =\nu(l / a)^{2} & \text { for } a>l \text { (high energy) }
\end{array}
$$

The radius of gyration is given by $a=R / B$ where the rigidity $\mathrm{R}=\mathrm{Pc} / \mathrm{Ze}$ and $\mathrm{B}=$ magnetic flux density. Using this and equation (5) we can rewrite equation (4) as follows:

$$
\begin{aligned}
\frac{d j}{d E_{n}} & =\left(\frac{d j}{d E_{n}}\right)_{\infty} \exp \left[-\left(\frac{3 V \nu}{c}\right)^{1 / \beta}\right] \quad \text { for } a<l \quad \text { (low energy), } \\
& =\left(\frac{d j}{d E_{n}}\right)_{\infty} \exp \left[-\left(\frac{3 W}{c} l^{2} B^{2}\right) 1 / \beta R^{2}\right] \text { for } a \gg l \text { (high energy). }
\end{aligned}
$$

In the low energy case the exponent varies as $1 / \beta$; this is therefore a velocity dependent modulation. At higher energies the exponent has a $1 / \beta R^{2}$ dependence. If, in fact, the kinetic energy is larger than the rest energy of the cosmic ray particle we can make the approximation that 
$\beta \cong 1$. The exponent therefore varies approximately as $1 / R^{2}$. This then is a rigidity dependent modulation. .

In interplanetary space there will be a distribution of scale sizes, $\ell$, of the magnetic field irregularities. For a particle with a given radius of gyration, a, one will find some scattering centers with $\ell<a$ and some with $\ell>a$. Only in the extreme cases, i.e. at either very small or very large energies can we apply the above simplifications. For the extreme low energy case there is a further difficulty. If the particle's radius of gyration is much less than $l$ we can invoke the adiabatic approximation wherein a particle's guiding center simply follows the field line. The previous notions of fixed scattering centers in three dimensional space then no longer apply.

Dorman $[1960,1963]$ has proposed a model for the intermediate energy region wherein scattering centers both larger and smaller than the particle's radius of gyration exist. He has assumed that in this case the scattering mean free path $\lambda=a$, the radius of gyration. This assumption leads to a $1 / \beta R$ dependence for the modulation. The equation relating cosmic ray intensities inside and outside the sun's influence then becomes:

$$
\frac{d j}{d E_{n}}=\left(\frac{d j}{d E_{n}}\right)_{\infty} \exp \left[-\left(\begin{array}{ll}
\frac{3 V \nu}{c} & \ell B
\end{array}\right) 1 / B \bar{R}\right]
$$

We now ask what each of the foregoing special cases of Parker's solar modulation model predict regarding the behavior of the protonhelium ratio as a function of time. We recall that the measurements presented here were all taken at the same velocity or energy/nucleon. Referring to equation (4) we then see that the ratio of proton and helium intensities at the same energy/nucleon is given by: 


$$
\begin{aligned}
& \Gamma_{p, H e}=\frac{\left(d j / d E_{n}\right) p}{\left(d_{j} / d E_{n}\right) H e} \\
& =\frac{\left(d j / d E_{n}\right) p, \infty \exp \left[\frac{-3 V}{W} \int_{r_{e} \frac{d r}{\lambda_{p}}}^{\infty}\right]}{\left(d j / d E_{n}\right) H e, \infty \exp \left[\frac{-\frac{3 V}{W}}{d_{r_{e}}^{\infty} \frac{d r}{\lambda_{H e}}}\right]} \\
& =\left(\Gamma_{\mathrm{p}, \mathrm{He}}\right)^{\infty} \exp \left[-\frac{3 \mathrm{~V}}{\mathrm{~W}} \int_{\mathrm{r}}^{\infty}\left(\frac{1}{\lambda_{\mathrm{p}}}-\frac{1}{\lambda_{\mathrm{He}}}\right)\right]
\end{aligned}
$$

Again, we would like to express the result (8) in a form more closely related to the quantities that we actually measure. For the three special cases discussed previously the proton helium ratio can be written:

$$
\begin{aligned}
& \Gamma_{\mathrm{P}, \mathrm{He}}=\left(\Gamma_{\mathrm{p}, \mathrm{He}}\right)_{\infty} \quad \Gamma_{0<l} \quad \text { (low energy), } \\
& \Gamma_{\mathrm{p}, \mathrm{He}}=\left(\Gamma_{\mathrm{p}, \mathrm{He}}\right)_{\infty} \exp \left[-\left(\frac{3 \mathrm{~W}}{\mathrm{c}} \ell \mathrm{B}\right) 1 / \beta\left(1 / \mathrm{R}_{\mathrm{p}}-1 / \mathrm{R}_{\mathrm{He}}\right)\right] \\
& \text { al (medium energy), } \\
& \Gamma_{\mathrm{p}, \mathrm{He}}=\left(\Gamma_{\mathrm{p}, \mathrm{He}}\right)_{\infty} \exp \left[-\left(\frac{3 W}{c} \ell^{2} \mathrm{~B}^{2}\right) 1 / B\left(1 / \mathrm{R}_{\mathrm{p}}{ }^{2}-1 / \mathrm{R}_{\mathrm{He}}{ }^{2}\right)\right] \\
& \text { a }>\text { l (high energy), }
\end{aligned}
$$

For the low energy case $(a<<)$ the exponents in the proton and alpha particle modulation expressions are identical in a velocity (energy/nucleon) representation. The exponents therefore cancel in the proton-alpha ratio and the ratio observed at the earth's orbit is the same as that in local interstellar space. Furthermore, it is a reasonably safe assumption that the proton-alpha ratio in local interstellar space is constant in time at least over the relatively short time scale relevant to this experiment. Therefore, a velocity dependent modulation mechanism predicts that the proton-alpha ratio at the earth's orbit remains constant in time.

Since at the same velocity the proton and alpha particle rigidities are unequal $\left(R_{\alpha}(\beta)=2 R_{p}(\beta)\right)$ the exponents at medium and high energies do 
not cancel. As one approaches solar maximum and modulation of the galactic cosmic rays increases the quantities $\frac{3 W}{C} \ell B$ and $\frac{3 V \nu}{c} l^{2} B^{2}$ will become larger. One therefore expects that in the energy regions where equations (10) and (11) are valid the proton alpha ratio will decrease with increasing modulation.

It is entirely possible that the scale size, $l$, of the magnetic irregularities can change over a solar cycle. When interpreting data at a single velocity one must therefore allow for the possibility that the nature of the modulation mechanism (e.g. velocity dependent or rigidity dependent) could also change with varying degrees of modulation.

We now refer again to Figure 22 and reexamine the data on the basis of the previous discussion. The proton-alpha ratio from 1963 to 1965 is constant within the experimental errors. The data for these years is therefore consistent with a velocity dependent form for the solar modulation. Other investigators [Gloeckler, 1965; Balasubrahmanyan, et al., 1966] have in fact reported agreement with the velocity dependent model during these years. The proton-alpha ratio at $85 \mathrm{MeV} / \mathrm{nucleon}$ during the period from 1963-1965 had a value of approximately 4.0. The measurement of McDonald and Webber [1964] in 1959 placed an upper bound of 1.0 on this ratio. Even with the large experimental errors this is clearly not consistent with the measurements in 1963-1965. We therefore conclude that the nature of the modulation mechanism must have changed sometime between 1959 and 1963. Unfortunately, the experimental errors are too large to permit a more accurate determination of exactly when this transition occurred. The 1962 measurement falls somewhat below the values for 1963-1965 and provides a weak indication that the transition began at this time. Assuming that this is true one would 
then expect that the 1961 proton-alpha ratio would be further reduced. It is particularly unfortunate that the errors in the 1961 measurement are too large to test this hypothesis.

Courtier and Linney [1965] have plotted the proton-helium ratio from 1959 to 1965 at higher values of kinetic energy/nucleon than those discussed here. At these energies a similar trend was observed. The proton-helium ratio was also observed to rise with decreasing solar modulation.

C. Solar Modulation in the 80-500 MeV/nucleon Interval from 1963 to 1965.

The modified version of the detector was used only in the final year of the balloon flight series, 1965. Consequently data at higher energies were obtained only in 1965. The primary helium spectrum measured at Fort Churchill in June, 1965 is shown in Figure 23. The lowest energy point is at $65 \mathrm{MeV} /$ nucleon. Atmospheric absorption prevented measurements below this energy. Also plotted are the data of Balasubrahmanyan, et a1. [1966] from 30 to $750 \mathrm{MeV} /$ nucleon. The low energy portion ( $30-70 \mathrm{MeV} /$ nucleon) was obtained from the IMP III satellite data. The high energy portion was obtained from a double $\mathrm{dE} / \mathrm{dx}$ and Cerenkov telescope which was flown in the same balloon payload along with the $\mathrm{dE} / \mathrm{dx}$ and $\mathrm{E}$ detector. It is clear that the independent sets of data are in quite good agreement.

Previously we analyzed the solar modulation of galactic cosmic rays as a function of time at a single energy. We now adopt a different method of analysis and explore the dependency of the solar modulation on particle energy/nucleon (or velocity) during a fixed time interval, i.e. we will hold time constant and let energy vary. Again, the theoretical 
basis will be the various special cases of Parker's mode1. For convenience, the modulation equations can be rewritten in the following form:

$$
\frac{d j}{d E_{n}}=\left(\frac{d j}{d E_{n}}\right)_{\infty} \exp [-K(t) f(\beta, R)],
$$

where

$$
\begin{aligned}
f(\beta, R) & =1 / \beta \text { low energy, } \\
& =1 / \beta R \text { medium energy, } \\
& =1 / \beta R^{2} \text { high energy. }
\end{aligned}
$$

The method of analysis is similar to that used by Gloeckler [1965], Webber [1965], and Silberberg [1966]. Consider the ratio of intensities at times $t_{I}$ and $t_{2}$. It is easily shown that this ratio is given by:

$$
\begin{aligned}
\ln \left[\frac{\left(d j / d E_{n}\right)_{t}=t_{2}}{\left(d j / d E_{n}\right) t=t_{1}}\right] & =-\left[K\left(t_{2}\right)-K\left(t_{1}\right)\right] f(\beta, R) \\
& =-\Delta K f(B, R) .
\end{aligned}
$$

Earlier it was mentioned that it is possible for the form of the modulation mechanism, i.e. $f(B, R)$, to change during the course of a solar cycle. In our differential analysis we would like to insure that $f(B, R)$ does not change from $t_{1}$ to $t_{2}$, since such a change would make any meaningful interpretation of the data extremely difficult. If we take $t_{2}-t_{1}=\Delta t<<$, where $T$ is the period of the solar modulation $(T \cong 11$ years), it is then probable that $f(\beta, R)$ will not change. However, if $\Delta t$ is small, the fractional change in intensity will also be small. The experimental errors will then become increasingly important and eventually also prevent any meaningful analysis. We shall therefore compromise and take $t_{1}=$ January, 1964 and $t_{2}=$ June, 1965. The increment in time, $\Delta t=1.5$ years, is small compared to the eleven year 
period of the solar modulation. Also, the behavior of the proton-helium ratio indicates that a single modulation mechanism was in effect, at least at $85 \mathrm{MeV} /$ nucleon during this time interval.

We are confronted with the problem of data selection. In 1963 a number of different measurements of the primary intensity in the 100-500 $\mathrm{MeV} /$ nucleon interval was made [Ormes and Webber, 1964; Balasubrahmanyan and McDonald, 1965; Freier and Waddington, 1965]. We shall arbitrarily choose the data from only one measurement, that of Balasubrahmanyan and McDonald [1965]. Our only criterion will be that the same experimental apparatus was used both in 1963 and 1965. The data of Balasubrahmanyan and McDonald satisfy this requirement. Since we will be considering only the fractional change in intensity, systematic errors will tend to cancel if data from the same experimental apparatus are always used. Similarly in the energy region below $100 \mathrm{MeV} /$ nucleon we will use the helium data of Gloeckler [1965] and Gloeckler and Jokipii [1966] and the proton data of McDonald and Ludwig [1964] and Balasubrahmanyan, et al. [1966].

These data are presented in Figure 24. A small difficulty arises since the 1963 data of Balasubrahmanyan, et al. were taken during June 1963. We have used the regression curves of Webber [1963] to extrapolate these data to January 1964 and make them directly comparable to the data below $100 \mathrm{MeV} /$ nucleon.

In Figure $24 \ln \left[\left(d j / d E_{n}\right)_{t}=t_{2} /\left(d j / d E_{n}\right)_{t}=t_{1}\right]$ is plotted as a function of the particle velocity, $\beta$. The ratio of intensities decreases with velocity as is expected. It appears that the modulation of the helium intensity is slightly greater than the modulation of the proton intensity especially at very low energies. The various modulation functions are also plotted in Figure 24; the value of $\Delta \mathrm{K}$ has been obtained by 
normalization at $\beta=0.8$ in all cases. The medium and high energy expressions $\left(1 / \beta R\right.$ and $\left.1 / \beta R^{2}\right)$ clearly disagree with the data. First, the slopes are inconsistent; and second, the predicted relative modulation of protons and helium disagree. The medium and high energy expressions predict that the fractional change in intensity will be larger for protons than it will be for helium. The data indicate that, in fact, the fractional change in the proton intensity is slightly less than that for the helium intensity.

The low energy expression $(1 / \beta)$ appears to provide a fairly good fit to the proton data. The helium data, however, appear to have a slightly steeper slope. At the same velocity the radius of gyration of an alpha particle will be twice that for a proton. Consequently it is more likely that the helium nucleus will undergo rigidity dependent scattering from irregularities in the interplanetary magnetic field. It is then possible that the helium data may, in fact, have a steeper slope than the proton data.

In a more recent paper Gloeckler and Jokipii [1966] have reported agreement with a $1 / \beta R$ dependence for the solar modulation using data from the IMP's I, II, and III satellites. This is to be contrasted with the earlier paper by Gloeckler [1965] where a velocity dependent $(1 / \beta)$ modulation was found using only the data from the IMP I satellite. Based on data from a large area Cerenkov-scintillator telescope, Webber [1966] has reported a $1 / \beta$ or $1 / R$ dependence for the modulation. However, as Gloeckler and Jokipii [1966] pointed out, Webber's data is really more consistent with a $1 / \beta R$ modulation. It is clear then that discrepancies exist between the various experimental results. There is consequently 
a real need for further more accurate measurements during the succeeding phases of the solar cycle. 


\section{ATMOSPHERIC SECONDARIES AND RE-ENTRANT ALBEDO}

\section{A. Atmospheric Secondaries}

A completely empirical approach has been adopted for obtaining the atmospheric secondary correction to our data. Methods used in the past have generally involved directly measuring the production of secondaries in nuclear emulsions and then by various means relating this to the production of secondaries in air. The method used here involves simultaneous balloon and satellite measurements of the low energy cosmic ray intensity, and provides a completely independent determination of the atmospheric secondary contribution.

The intensity of downward moving particles in the atmosphere can be written as follows:

$$
I(X)=I_{p}(X)+I_{s}(X)+I_{r a}(X),
$$

where

$$
\begin{aligned}
& I_{p}=\text { primary intensity, } \\
& I_{s}=\text { secondary intensity, } \\
& I_{r a}=\text { re-entrant albedo intensity, } \\
& X=\text { depth in atmosphere. }
\end{aligned}
$$

Re-entrant albedo consists only of particles with energies below the local geomagnetic cut-off. Since the geomagnetic cut-off for protons at Fort Churchill is less than $20 \mathrm{MeV}$, re-entrant albedo at this location can be neglected. Our detector measures the intensity I(X) between 20 and $80 \mathrm{MeV} /$ nucleon. Thus, if the primary intensity $I_{p}(X)$ is known, the secondary intensity is uniquely determined. We obtain the primary intensity at the top of the atmosphere, $I_{p}(0)$, from the IMP III satellite measurements of Balasubrahmanyan, et al. [1966]. It is then a straightforward matter to obtain the value of $I_{p}(X)$ at $X=3.1 \mathrm{gm} / \mathrm{cm}^{2}$, 
the altitude of the 1965 balloon (including $.3 \mathrm{gm} / \mathrm{cm}^{2}$ air equivalent of material in the balloon gondola). The difference in energy intervals is easily calculable, and a small correction must be made for nuclear interactions in the $3.1 \mathrm{gm} / \mathrm{cm}^{2}$ of atmosphere.

The IMP III spectrum (after passage through $3.1 \mathrm{gm} / \mathrm{cm}^{2}$ of absorber), the total spectrum at the detector, and the resultant secondary spectrum at the detector are shown in Figure 25. The surprising feature about the secondary spectrum is that it is essentially flat in the 20-80 $\mathrm{MeV} /$ nucleon interval. Also, we note that the secondary intensity is of the same order as the primary intensity at this particular depth in the atmosphere. Solar minimum is, of course, the optimum time for separation of primaries from background, since the primary intensity is at a maximum and effectively the signal to noise ratio is maximized. One expects that near solar maximum the situation will become much worse since the low energy primaries will be suppressed much more than the background.

Through averaging the two flights (65-1 and 65-2) it was also possible to obtain an intensity vs. altitude curve in the $20-80 \mathrm{MeV} / \mathrm{nucleon}$ interval. Again using the IMP III data, the primary intensity as a function of depth in the atmosphere was obtained. These were then subtracted to give the secondary intensity vs. altitude curve shown in Figure 26. Satellite data at low energies are also available for 1964 [McDonald and Ludwig, 1964]. Therefore it was possible to use the same method to obtain the 1964 secondary intensity. This was done only at an atmospheric depth corresponding to the floating altitude of the balloon and is also plotted in Figure 26. The agreement between the 1964 and 1965 secondary intensities provides a good internal consistency check. 
Two flights were made at low latitudes. The first (Flight 61-2) was launched in 1961 from Sioux Falls, S.D. The second (Flight 64-4) was launched in 1964 at approximately the same geomagnetic latitude from Fairibault, Minn. The total intensity at the detector for each of these flights is also plotted in Figure 26. It is significant that no measurable change in intensity at this latitude took place between 1961 and 1964. The geomagnetic cut-off at the latitude of Flights 61-2 and 64-4 is approximately $900 \mathrm{MeV}$ for protons [Shea and Smart, 1966]. Clearly the intensities observed in these flights in the 20 to $80 \mathrm{MeV}$ interval must then be entirely due to atmospheric secondaries and re-entrant albedo.

From our measurements an upper limit of $13 \%$ can be placed on the fractional change in intensity in the 20 to $80 \mathrm{MeV} /$ nucleon interval at the latitude of Sioux Falls from 1961 to 1964. In the discussion following, we shall argue that the secondary intensity at Fort Churchill is essentially the same as the secondary intensity at Sioux Falls, and that consequently the secondaries at Fort Churchill also remained constant from 1961 to 1964. Effectively, it is necessary to show that primaries with energies less than $900 \mathrm{MeV}$ (the geomagnetic cut-off at Sioux Falls) do not significantly contribute to the secondary intensity at Fort Churchi11. Our argument is based on the time variation of the primary intensity at the latitude of Sioux Falls due to solar modulation. The effect of increasing solar modulation is qualitatively similar to the effect of increasing geomagnetic cutoff. In both cases the low energy intensity is suppressed. In the case of solar modulation the energy dependency is gradual and continuous, whereas in the geomagnetic case the suppression occurs relatively suddenly at a single discrete energy, the geomagnetic cut-off. The differential proton intensity at $1 \mathrm{BeV}$ changed by more than a factor of two, [Webber, 1964] between 1961 and 
1964, yet no change was observed in the secondary intensity at Sioux Falls. It is therefore unlikely that primary cosmic rays with energies in the vicinity of $1 \mathrm{BeV}$ have contributed significantly to the secondary intensity at Sioux Falls. It is therefore even less likely that primary particles with energies less than 1 BeV have made a significant contribution to the secondary intensity at Fort Churchill. We shall then assume that the secondary intensity at Fort Churchill is the same as that at Sioux Falls.

As a corollary to this argument we claim that the median primary energy for the production of secondaries in the 20 to $80 \mathrm{MeV} /$ nucleon interval must be substantially higher than $1 \mathrm{BeV}$. This hypothesis is partially confirmed by the results of Flight $64-5$ which was launched from Palestine, Texas where the geomagnetic cut-off is $3.6 \mathrm{BeV}$ for protons. A tota 1 proton intensity of $.28 \pm .03$ particles $/ \mathrm{m}^{2}-\mathrm{MeV}-\mathrm{sec}-\mathrm{ster}$ was observed at the detector. This is very nearly equal to one-half of the secondary proton intensity observed at a similar depth in the atmosphere at Fort Churchi11. (We will show later that the re-entrant albedo contribution is smal1.) Thus the median primary for the production of secondary protons in the 20-80 MeV/nucleon lies somewhere in the vicinity of the cut-off energy at Palestine, $3.6 \mathrm{BeV}$. The fractional change in integral intensity at Palestine (> $3.6 \mathrm{BeV}$ ) from 1961 to 1964 was approximately $10 \%$ [Webber, 1964]. This is consistent with the upper bound of $13 \%$ placed on the fractional change in the low energy intensity at Sioux Falls from 1961 to 1964.

The possible existence of re-entrant albedo at Sioux Falls does not seriously affect the preceding conclusions. We observe a total flux at this latitude which remains constant from 1961 to 1964. One 
expects that re-entrant albedo and atmospheric secondaries will exhibit qualitatively similar long-term variations; that is, if the intensity of one component increases (or decreases), the intensity of the other will also increase (or decrease). Therefore if the sum of the two components remains constant, each individual component must also remain constant. We then conclude that within the limits of accuracy of our experiment the secondary intensity in the $20-80 \mathrm{MeV} /$ nucleon interval did not change from 1961 - 1964. On the basis of this argument we have simply applied the same secondary correction obtained from the simultaneous balloon and satellite measurements in 1965 to al1 of our measurements in the 1961 to 1965 period.

As we have mentioned, a number of different approaches have been adopted by various experimenters to obtain the proton secondary correction. Vogt [1962] and Brunstein [1964] have done quasi-theoretical calculations of the secondary intensity. Both calculations made use of the spectrum of secondary reaction products measured in emulsions. A somewhat different method has been used by Waddington and Freier [1964], Fichtel, et al. [1965], and others. The method involves a measurement more directly related to the atmospheric secondary intensity. Typically, an emulsion stack is scanned along a line which is $1 \mathrm{~cm}$ below the surface of the emulsion. At this depth in the emulsion both the primary spectrum and the spectrum of secondaries produced in the $1 \mathrm{~cm}$ of thickness of emulsion are measured. The emulsion secondaries are uniquely identified by tracing the tracks back to the interaction. It is claimed that the secondaries produced in $1 \mathrm{~cm}$ of emulsion will be the same as those produced in an equivalent thickness of air (approximately $3 \mathrm{gm} / \mathrm{cm}^{2}$ ). Our 1965 secondary spectrum was measured at an effective atmospheric depth of 
$3.1 \mathrm{gm} / \mathrm{cm}^{2}$. Evidence discussed earlier indicated that this spectrum did not change significantly from 1961 to 1965. We may therefore directly compare our secondary spectrum from $3.1 \mathrm{gm} / \mathrm{cm}^{2}$ of air to that obtained in 1963 and 1964 by Waddington and Freier $[1965,1965 \mathrm{a}]$ for a $3 \mathrm{gm} / \mathrm{cm}^{2}$ air equivalent thickness of emulsion. This is done in Figure 27.

In emulsion spectrums of the type presented in Figure 27, no distinction is usually made between protons and deuterons. At these low energies the energy is determined by measuring the particle's range in the emulsion. Consequently, in addition to our proton secondary spectrum, we have included in Figure 27 a spectrum representing the sum of the secondary proton and deuteron intensities with the addition made on an equal range basis. This should be directly comparable to the emulsion secondary spectrum. Unfortunately, the overlap between our data and those of Waddington and Freier is not large. It appears that, in the energy interval in which comparison can be made, our spectrum is somewhat flatter than that of Waddington and Freier. The difference, however, is not large and could be attributed to experimental uncertainties except, perhaps, at very low energies.

A detailed theoretical treatment of the production of secondaries in air, emulsion, or other absorbers is in general quite a complex problem. Extensive Monte Carlo calculations of intra-nuclear cascades have been carried out by Metropolis, et al. [1958a,b] and by Bertini [1963a,b; 1966]. Comparisons have been made between their results and the limited available data. In general, agreement was found between the experimental and theoretical results.

In order to derive a theoretical ratio between the secondary spectrums in emulsion and in air, it is necessary to know the number of 
cascade nucleons as a function of the incident particle's energy and also to know the energy spectrum of the cascade nucleons themselves. Fichtel, et al. [1964] have assumed that the cascade nucleon spectrums in air and in emulsion are the same; and they have integrated the data of Metropolis, et al. over the incident cosmic ray spectrum. They obtained a ratio of 0.85 between secondaries from air and secondaries from emulsion. In the region of overlap this ratio is consistent with the data presented in Figure 27. As mentioned earlier, however, the slopes of the emulsion and air spectrums appear to be different at low energies. Metropolis, et a1. have calculated the secondary proton spectrums for aluminum and uranium target nuclei with incident proton energies of 460 and $1840 \mathrm{MeV}$. For both incident energies the cascade proton spectrums from aluminum were slightly flatter at low energies than were the spectrums from uranium. This behavior is qualitatively similar to that exhibited in Figure 27. Also, if one integrates the cascade spectrum given by Metropolis, et a1. for $1840 \mathrm{MeV}$ protons incident on aluminum over 3 $\mathrm{gm} / \mathrm{cm}^{2}$ of air, a flat spectrum is obtained, similar in shape to that shown in Figure 27.

Also plotted in Figure 27 is the quasi-theoretical secondary correction of Vogt [1962]. Obviously there is a serious disagreement between the secondary spectrum obtained in this work and that obtained by Vogt. His results predict a secondary spectrum at $5 \mathrm{gm} / \mathrm{cm}^{2}$. Since the secondary intensity at this altitude increases with depth in the atmosphere these results are quite incompatible. For this reason we feel that the rise in the spectrum observed by Vogt at low energies ( $5150 \mathrm{MeV}$ ) is mainly due to atmospheric secondaries. An underestimate of the atmospheric secondary correction, however, cannot explain the time variation observed 
by Meyer \& Vogt [1963] from 1960 to 1961. A decrease in intensity was observed which was in positive correlation with the sunspot cycle. Our primary proton and helium data exhibited no such behavior (see Figure 21). However, our data go back only as far as 1961 . We therefore cannot exclude the possibility of a small quiescent solar contribution to the intensity at low energies in 1960 .

\section{B. Re-entrant Albedo}

Since the geomagnetic cut-off excludes low energy primaries at the latitude of Flight $64-4$ the total intensity measured during this flight can be written:

$$
I(X)=I_{S}(X)+I_{r a}(X)
$$

where $X=5.0 \mathrm{gm} / \mathrm{cm}^{2}$. Therefore, if the secondary intensity at this latitude $I_{S}(5.0)$ is known, we can determine the re-entrant albedo. We know the secondary intensity at Fort Churchill where the geomagnetic cutoff is less than the atmospheric cut-off. However, our previous arguments indicated that the secondary intensities at Fort Churchill and Sioux Falls were the same. Thus by a simple subtraction of these intensities we can obtain the re-entrant albedo intensity at Sioux Falls.

Reference to Figure 26 shows that there is no measurable difference between the total intensity at the latitude of Sioux Falls and the secondary intensity at Fort Churchill. Statistical uncertainties then permit us to place an upper bound on the re-entrant albedo at $5 \mathrm{gm} / \mathrm{cm}^{3}$ at Sioux Falls of .11 protons $/ \mathrm{m}^{2}-\mathrm{MeV}-\mathrm{sec}-\mathrm{ster}$. Correction to the top of the atmosphere yields an upper limit of .18 protons $/ \mathrm{m}^{2}-\mathrm{MeV}-\mathrm{sec}-\mathrm{ster}$ at an average energy of $95 \mathrm{MeV}$. In Figure 28 we have plotted the re-entrant albedo spectrum of Webber [1964] at $5 \mathrm{gm} / \mathrm{cm}^{2}$ along with the upper bound 
obtained in this work. Also plotted is a point obtained by Ray [1966] from a theoretical calculation. The difference between our upper bound and Webber's spectrum is not so great that it could not be attributed to experimental error. Webber has, however, in the same context obtained an atmospheric secondary spectrum which is substantially less that that presented in this work. Since the atmospheric secondaries and re-entrant albedo are by virtue of the experimental method mutually interdependent results, we feel that a real discrepancy exists. 
Previously we have discussed the production of one of the hydrogen isotopes, $\mathrm{H}^{1}$ (protons) in the earth's atmosphere. We will now present data on the production of the other two known isotopes of hydrogen, $\mathrm{H}^{2}$ and $\mathrm{H}^{3}$ (deuterium and tritium) in the earth's atmosphere. Certain very rare radioactive isotopes found in the earth's atmosphere are thought to be produced by interactions of primary cosmic rays with the atmosphere. It is assumed that an equilibrium is maintained between the production of such isotopes by cosmic radiation and their loss by radioactive decay and escape from the stratosphere. Perhaps the most famous of these isotopes is $\mathrm{C}^{14}$ with a half-1ife of 5568 years. In certain isolated samples of carbon - for example, fossils buried in the earth - the equilibrium process is destroyed. No recycling with the atmosphere takes place and consequently only the loss mechanism due to radioactive decay is present. By measuring the $\mathrm{C}^{14}$ concentration in such samples it is possible to determine their ages. The long half-life of $\mathrm{C}^{14}$ permits the determination of ages of samples many thousands of years old. Tritium falls into the class of rare radioactive elements thought to be produced by cosmic ray interactions in the earth's atmosphere. It's half-life of 12.5 years is substantially shorter than that of $\mathrm{C}^{14}$. Consequently its usefulness is limited to hundreds rather than thousands of years in the past. It is possible, however, to obtain a much more detailed picture of recent events using tritium. The pre-hydrogen bomb abundance of tritium is quite smal1. Typically, measurements have recorded a tritium to hydrogen ratio of one part in $10^{18}$ in the earth's atmosphere. Unfortunately, thermonuclear tests completely destroyed the 
tritium balance in the atmosphere. The "Castle" tests increased the atmospheric tritium abundance by several orders of magnitude. In the following discussion we will therefore necessarily always refer to the pre-bomb abundance measurements.

There have previously been no direct measurements made of the production of tritium in the earth's atmosphere. Several calculations of the tritium production rate in the atmosphere have been made [Fireman and Rowland, 1955; Currie, et a1., 1956; Benioff, 1957; La1 and Peters, 1962] based on machine measured cross-sections for the production of tritium by nucleons in air. The production rate, $P$, for tritium is defined as the total number of tritons produced per second in a $1 \mathrm{~cm}^{2}$ column of atmosphere. To calculate $P$ further information is required on the variation of the interaction rate with depth in the atmosphere. Generally the calculations have used emulsion data on star production as a function of atmospheric depth. One is interested in obtaining a global average production rate to compare with the global inventory of tritium. Due to geomagnetic cutoff effects the production rate will vary with latitude. Again, emulsion star data as a function of geomagnetic latitude is used to obtain a global average.

The results of the calculations described above 1ie between $P=.14$ and $\mathrm{P}=.37$ tritons $/ \mathrm{cm}^{2}-\mathrm{sec}$ (global average). Unfortunately, some difficulties arise when attempting to compare the calculated production rate with the measured terrestrial abundance of tritium. The tritium content has been found to vary with the type of sample on which the measurement is made, for example, oceanic rain water, continental rain water, glacial ice, vintage wines. The question arises as to which type of sample is most truly representive of the equilibrium abundance of tritium. La1 
and Peters [1962] claim that their calculated cosmic ray production rate is consistent with the equilibrium abundance of tritium. However, certain other authors [Craig, 1957; Begemann and Libby, 1957; Begemann, 1959] find that the cosmic ray production rate is insufficient to maintain the global abundance of tritium. It has been suggested that the sun might also be a source of tritium [Craig, 1957; Simpson, 1960] either directly by accretion or indirectly by interaction of high energy solar flare particles with the earth's atmosphere. One can only say that the question of the tritium balance is still open and further work is needed.

The 1965 balloon flights exhibited particularly good resolution in the hydrogen region. Circuitry of extended dynamic range permitted accumulation of data in the vicinity of the proton line for a longer period of time than was possible in the earlier years of the balloon flight series. As a result, a well resolved deuteron line was obtained, and it was also possible to resolve a triton line. In Figure 29 mass histograms for the two flights at Fort Churchill in 1965 are plotted. The histograms were constructed in exactly the same manner as those described in Chapter II, with one exception. Instead of taking the proton line as a reference the deuteron line was used. No line drawn parallel to the deuteron line will coincide exactly with the triton line. However, the difference is small enough that no significant artificial overlapping of the deuteron and triton distributions will occur. One can see from Figure 29 that in flight 65-1 deuterons and tritons are quite we11 separated. In flight 65-2 the resolution is not quite as good, owing to poorer statistics and perhaps to small gain shifts. 
The hydrogen isotope data obtained in 1965 are summarized in Table III. Again, we have compared intensities on an energy/nucleon basis. Due to the widely differing charge to mass ratios of the hydrogen isotopes, we are limited to the 20-38 MeV/nucleon interval. This is the widest energy interval in which it was possible to obtain data on all three hydrogen isotopes. It is our contention that nearly al1 the deuterons and tritons we observe are atmospheric secondary reaction products. It is possible that there is a small amount of primary deuterium present. Fan, et al. [1966] have reported the presence of a small flux of primary deuterium in their IMP II satellite measurements. The deuteron intensity measured by them was only 4 to 5 per cent of the primary proton intensity and was very near the limit of resolution of their detector. Hagge, et a1. [1966], on the other hand have reported the absence of deuterium in the primary cosmic radiation. If the results of Fan, et al. are correct and there really is primary deuterium, our conclusions will still not be affected. The small intensity observed by them would be even further reduced after passage through $3 \mathrm{gm} / \mathrm{cm}^{2}$ of air. It would then become negligibly small compared to the intensity we measure. We will therefore assume that a11 deuterons measured by our detector are atmospheric secondaries.

The energetic tritons observed in our experiment, if primary, could be either galactic or solar. The 12.5 year half-life eliminates the possibility of galactic origin. Since no appreciable solar activity was present during flights $65-1$ and $65-2$ we may also eliminate solar origin. A11 tritons we observe must therefore be atmospheric secondaries. Since we are dealing with atmospheric secondaries, it is appropriate to compare the deuteron and triton intensities with the secondary proton intensity at the detector. The deuteron production was quite large, approximately 
$-70-$

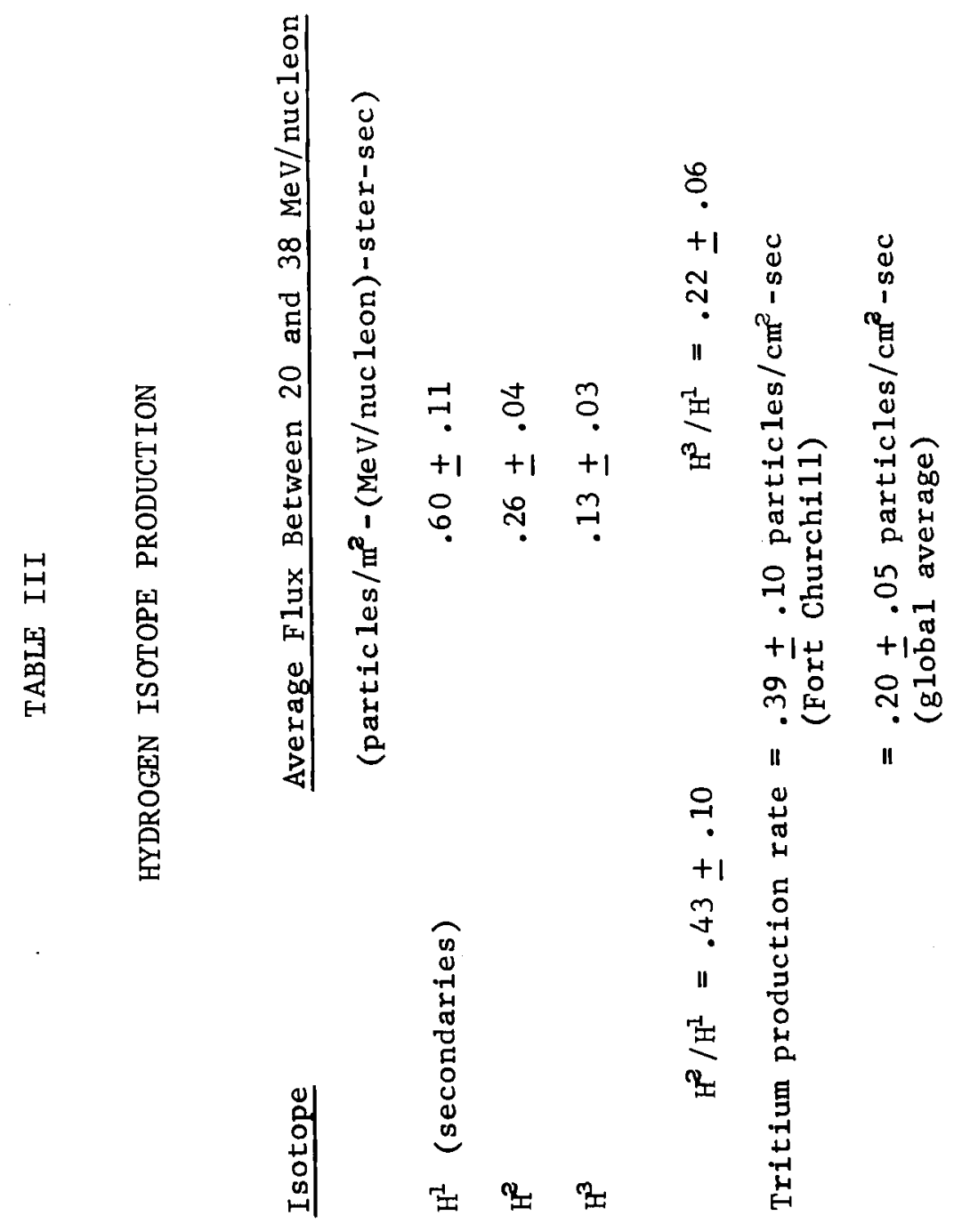


half of the secondary proton production. The tritium intensity was also surprisingly large. A triton to secondary proton intensity ratio of . 22 was observed. Our deuteron to proton ratio is consistent with the data obtained from secondary reaction products in nuclear emulsions. Dahanayake, et a1. [1955] have reported a deuteron to proton ratio of approximately $30 \%$ at $50 \mathrm{MeV} /$ nucleon in emulsions.

The question arises as to what type of interactions produced these particles. Two possibilities present themselves: (1) The energetic deuterons and tritons could be produced by the fragmentation of heavy primary cosmic ray nuclei as they impinge on the earth's atmosphere. (2) They could be the result of nuclear evaporation or pick-up reactions of the primary cosmic ray protons with air nucleii. Several arguments indicate that the latter possibility is most likely. First, when a heavy nucleus undergoes fragmentation, the secondary products tend to maintain the same velocity (or energy/nucleon) as the incident heavy particle. Therefore, most of the deuterons and tritons that we observe, which have energies less than $80 \mathrm{MeV} /$ nucleon, will be produced by heavier particles whose energies are also less than $80 \mathrm{MeV} /$ nucleon. The intensity of such low energy heavy nuclei, however, is much too sma1l to account for the large numbers of deuterons and tritons observed. The fact that little or no deuterium has been observed in the primary cosmic radiation, which has passed through approximately $3 \mathrm{gm} / \mathrm{cm}^{2}$ of interstellar matter agrees with this hypothesis. Second, the agreement of our deuteron to proton ratio with that measured in emulsions argues that nuclear evaporation or pick-up reactions are most important. Third, in Figure 30 we have plotted the deuteron intensity and the secondary proton intensity as a function of depth in the atmosphere. The qualitative agreement of the altitude 
dependency of these two hydrogen isotopes indicates that the production mechanisms are similar.

Cosmic ray produced deuterium contributes only a very small amount to the world's supply of deuterium. We have integrated the production rate of deuterium by cosmic rays as obtained from our data over the lifetime of the earth. During this time only approximately $10^{10}$ gm of deuterium were produced by cosmic rays. This is many orders of magnitude less than the global abundance of deuterium. Tritium is extremely rare and therefore a much more likely candidate. Based on our direct measurement of the secondary triton $f l u x$ at $3 \mathrm{gm} / \mathrm{cm}^{2}$ we shall therefore attempt to calculate the production rate of tritium in the earth's atmosphere.

Our measurement gives the triton intensity at a single energy, a single instant of time, a single position on the earth, and a single depth in the atmosphere. We must therefore obtain information elsewhere on the time, energy, latitude, and atmospheric depth dependency of the interactions producing tritium in order to obtain a global average production rate. Our source will again be the data on star production in nuclear emulsions.

In our calculations we shall follow a procedure out lined by Rossi [1952]. We shall define a source function for tritons as follows:

$S_{t}(E, x) d x d E=$ number of interactions per second producting a triton at an atmospheric depth $x$ in $d x$, and at energy $E$ in $\mathrm{dE}$ in the vertical direction.

It can then be shown that the vertical intensity of tritons at energy $E$ and depth $x$ in the atmosphere is given by [Rossi, 1952, page 487]:

$$
I_{t}(E, x)=\frac{1}{d E / d x} \int_{0}^{x} \frac{d E^{\prime}}{d x^{\prime}} S_{t}\left(E^{\prime}, x^{\prime}\right) e^{-\left(x-x^{\prime}\right) / \lambda} t d x^{\prime}
$$


where $\mathrm{dE} / \mathrm{dX}$ is the energy loss rate due to ionization in the atmosphere, and $\lambda_{t}$ is the collision mean free path for tritons in the atmosphere. The energies $E$ and $E^{\prime}$ are related as follows:

$$
R\left(E^{\prime}\right)=R(E)+x-x^{\prime} \text {, }
$$

where $R(E)$ is the range in air of particle with energy $E$.

We will be concerned with only small values of $x$ and $x^{\prime}\left(\leqslant 3 \mathrm{gm} / \mathrm{cm}^{2}\right)$; therefore the exponential term in equation (1) can be neglected.

Equation (1) can then be rewritten as follows:

$$
\begin{aligned}
I_{t}(E, x) & =\frac{1}{d E / d x} \int_{0}^{x} \frac{d E^{\prime}}{d x^{\prime}} S_{t}\left(E^{\prime}, x\right) d x^{\prime} \\
& =\frac{1}{d E / d x} \int_{E}^{E_{m}} S_{t}\left(E^{\prime}, x\right) d E^{\prime} .
\end{aligned}
$$

The upper limit $\mathrm{E}_{\mathrm{m}}$ is determined by the relation

$$
R\left(E_{m}\right)=R(E)+x
$$

We will make the assumption that the source function is a separable function of $E^{\prime}$ and $x^{\prime}$; that is, that $S_{t}\left(E^{\prime}, x\right)$ can be written

$$
S_{t}\left(E^{\prime}, x\right)=\text { const. } x \cdot S_{1}\left(E^{\prime}\right) S_{2}(x) \text {. }
$$

Physically, this is equivalent to assuming that the shape of the energy spectrum of secondary tritons at the point of interaction is not strongly dependent on depth in the atmosphere. For $S_{1}\left(E^{\prime}\right)$ we will use the emulsion star proton spectrum given by Powe11, Fowler, and Perkins [1959]. Since no triton spectrum is available, we must assume that protons and tritons have similar spectral shapes. Since we will be using a normalization procedure, our results will not depend too strongly on the choice of spectral shape. A piecewise power law fit has been made to the spectrum of Powe11, Fowler, and Perkins to simplify integration. For the depth dependency of the source function we have used the curves given by Lal 
and Peters [1962] based on emulsion star data.

Our measurement consists of determining the value of the triton intensity $I_{t}(E, x)$ at a single value of $E$ and $x$. After integration of equation (3) we can use this value of $I_{t}$ to solve for the constant in equation (5). Thus we have determined the triton source function $S_{t}\left(E^{\prime}, x\right)$. With this done it is a straightforward matter to calculate the tritium production rate. We have, however, obtained the source function only in the vertical direction. It is necessary to make some assumption about the angular dependency of the source function. Again we shall use emulsion star data. Brunstein [1963] has fit the emulsion data on the angular dependency of star protons [Camerini, et a1., 1951] with a function of the form

$$
f(\theta)=k e^{-3 \theta / \pi}
$$

where $\theta$ is the angle from the vertical.

To obtain the triton production rate at Fort Churchill we must then perform the following integration:

$$
P=\int_{\Omega}^{x} f(\theta) S_{t}\left(E^{\prime}, x\right) d E^{\prime} d x d \Omega
$$

where $x$ is the total thickness of atmosphere $\left(1030 \mathrm{gm} / \mathrm{cm}^{2}\right)$. A value of $.39 \pm .10$ tritons $/ \mathrm{cm}^{d}-\mathrm{sec}$ was obtained for the production rate at Fort Churchil1.

To obtain the global average tritium production rate we have used the curves of Lal and Peters giving the isotope production rate as a function of latitude. We obtain a value $\mathrm{P}_{\mathrm{avg}},=.20 \pm .05$. This is effectively also on average over a solar cycle. Our value lies well within the range of results $\left(.14-.31\right.$ tritons $\left./ \mathrm{cm}^{2}-\mathrm{sec}\right)$ from the quasitheoretical calculations of the production rate described earlier. In deriving the preceding results, we have used emulsion star data. 
Two assumptions have been made: that the secondary proton spectrums in emulsion and air are identical, and that the secondary proton, deuteron, and triton spectrums are also identical. The data presented in Chapter VI, however, indicated that a small difference existed between the spectrums of protons produced in emulsion and in air. It is possible to check the validity of these assumptions by examining the altitude dependency of the secondary proton and deuteron intensities as shown in Figure 30. We have integrated the intensity derived from equation 3 between 20 and $80 \mathrm{MeV}$, the energy range of the detector. These curves were then normalized to the proton and deuteron intensities at $3 \mathrm{gm} / \mathrm{cm}^{2}$ as measured in this experiment. The results of these calculations are shown by the solid curves in Figure 30. For protons, the calculated curve goes through a maximum higher in the atmosphere than the experimental data. This is, in fact, consistent with a flatter proton spectrum in air than in emulsion, as was reported in Chapter VI. The agreement between the deuteron data and the calculated curve is, however, quite good. The difference between the experimental proton and deuteron altitude curves can be explained by a slightly softer secondary deuteron spectrum. We are thus confronted with a fortuitous situation wherein the errors arising from our assumptions tend to cancel. 
A complete understanding of the eleven year modulation of galactic cosmic rays will come only through long-term detailed studies over the entire solar cycle. We have presented data from a relatively long series of balloon flights over a period of five years. Still, this covers less than one-half of one solar cycle. With the development of more sophisticated satellite technology, it has become possible to orbit multiparameter detectors similar to the one described in this thesis. Data from such detectors cover the period from late 1963 to the present. In addition to being free from the problem of atmospheric secondaries, these satellite-borne detectors provide a continuous monitor of the cosmic ray intensity over time periods that are long compared to the duration of an average balloon flight. The primary cosmic ray data presented in this thesis has been limited by statistics to only proton and alpha particle intensities. With the great volume of data accumulated during long-term satellite experiments it will become possible to study the time variations of other less abundant components of the cosmic radiation.

Thus far, our knowledge of the cosmic ray intensity is limited to a very narrow region about the orbit of the earth. Most theories of solar modulation predict a radial gradient in the cosmic ray intensity. Experiments aboard spacecraft whose trajectories carry them far from the earth's orbit could measure such a gradient. In addition magnetometers carried aboard these satellites could determine the radial dependence of the interplanetary magnetic field. Measurements such as these can be expected to provide a better understanding of the solar modulation. 
We have also presented data on the production of low energy secondaries in the earth's atmosphere. A proton secondary spectrum was obtained by comparing simultaneous balloon and satellite measurements. Satellite experiments capable of measuring the cosmic spectrum at higher energies are now being developed. Included among these is the modified $\mathrm{dE} / \mathrm{dx}$ and $\mathrm{E}$ detector which will be flown aboard the OGO-E spacecraft. It will thus be possible to extend the atmospheric secondary spectrum to higher energies.

Finally, we have experimentally determined the cosmic ray production rate of deuterium and tritium in the earth's atmosphere. The tritium loss rate, however, due to radioactive decay and escape from the stratosphere still remains an open question. Experimental work is now extremely difficult due to the great tritium production from thermonuclear tests the tritium abundance has been increased by approximately three orders of magnitude. The 12.5 year half-life of tritium means that it will take of the order of 100 years before equilibrium is restored given the unlikely possibility that no thermonuclear tests occur during this period. 


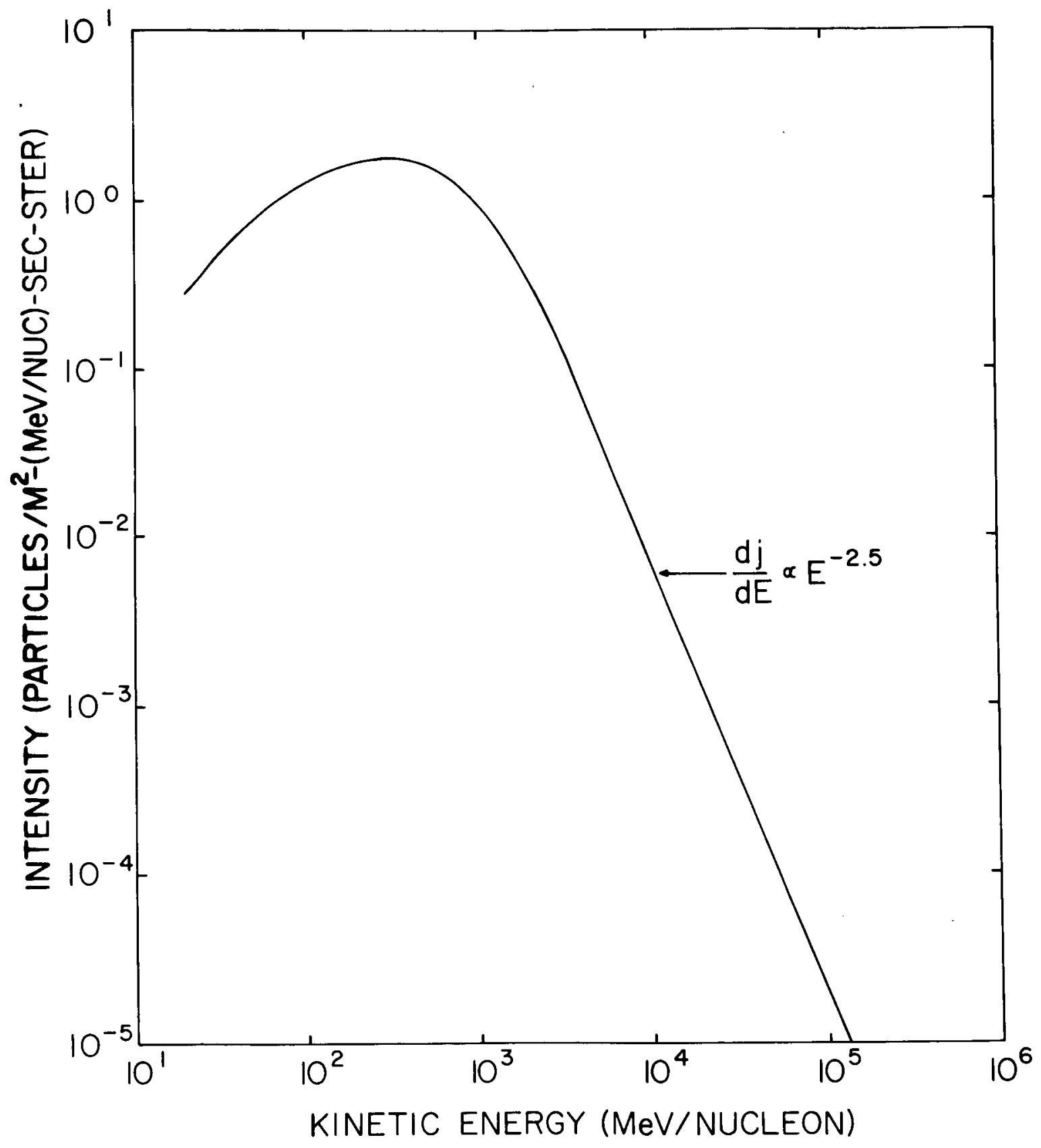

Figure 1. Total Cosmic Ray Spectrum at Solar Minimum 


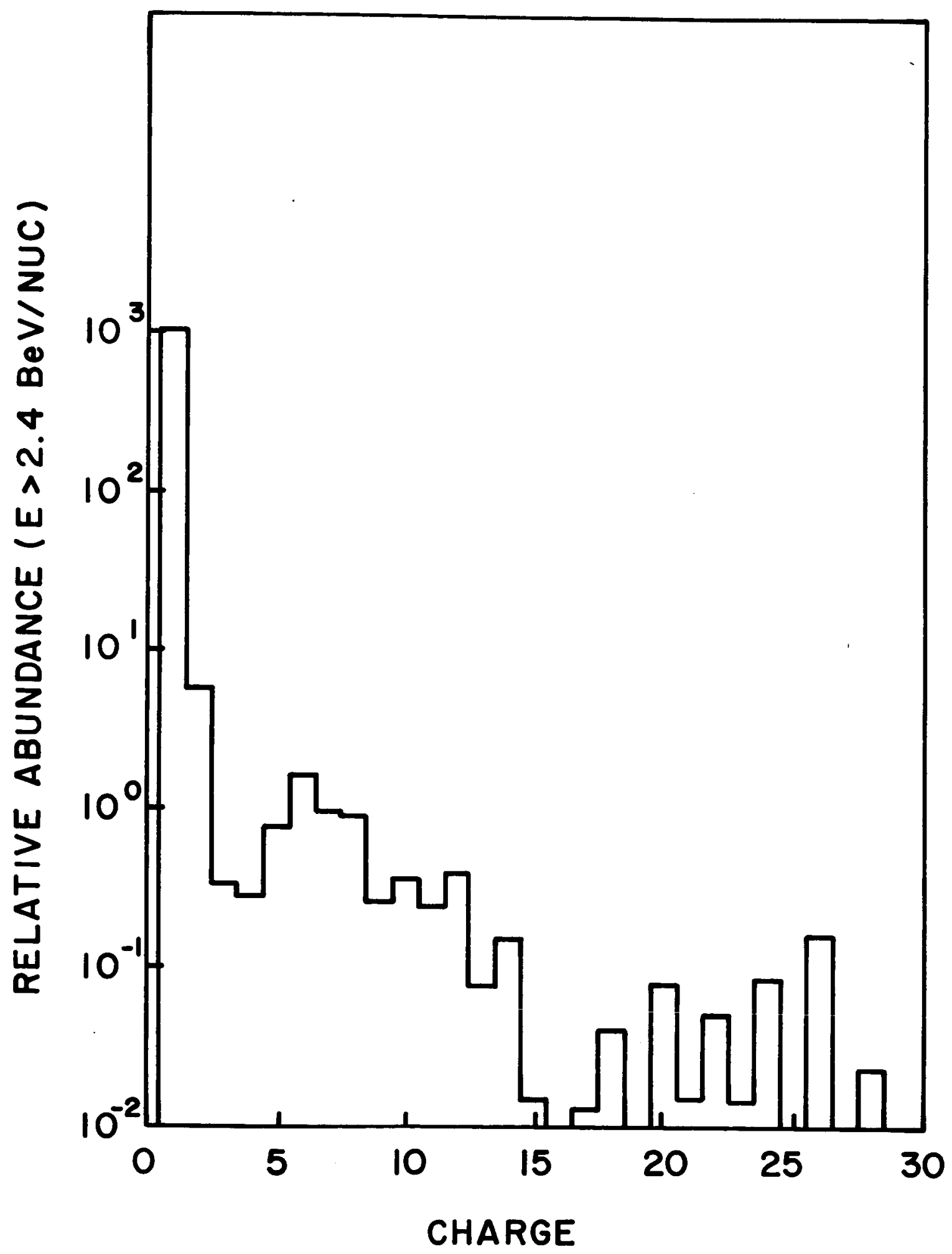

Figure 2. Cosmic Ray Relative Abundances 


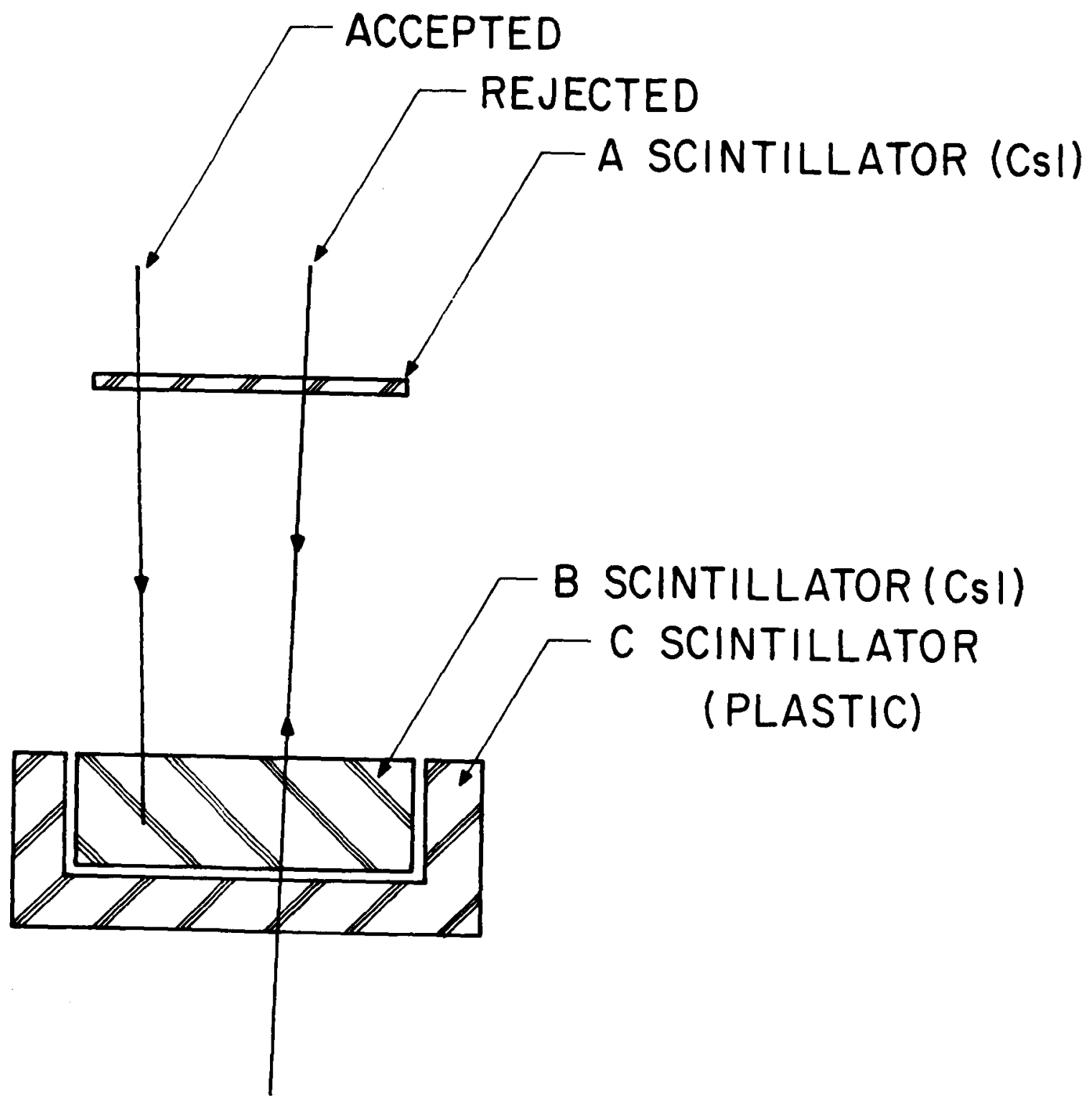

Figure 3. Idealized View of the DE/DX and E Detector 


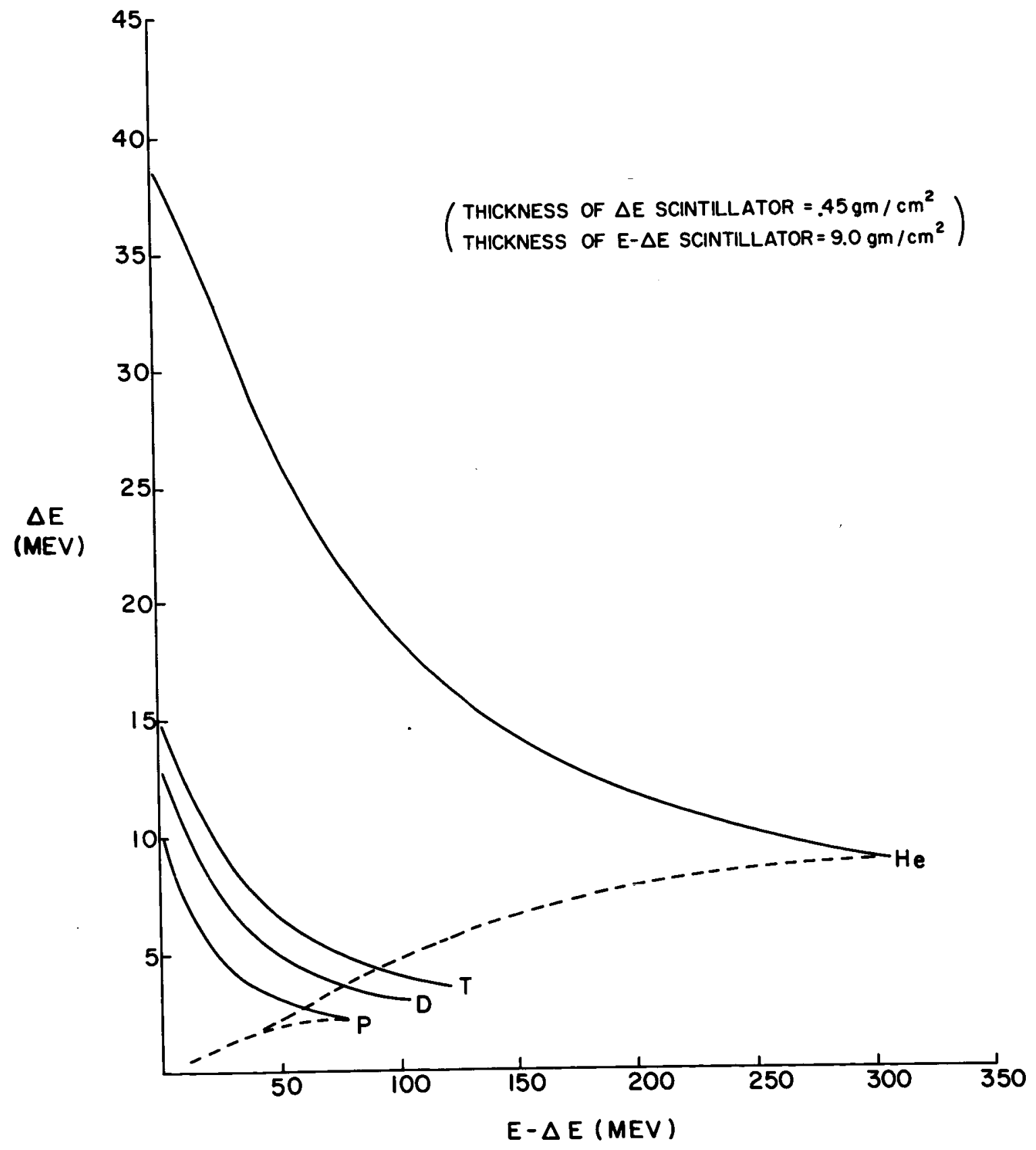

Figure 4. $\triangle E$ VS. $E-\Delta E$ 


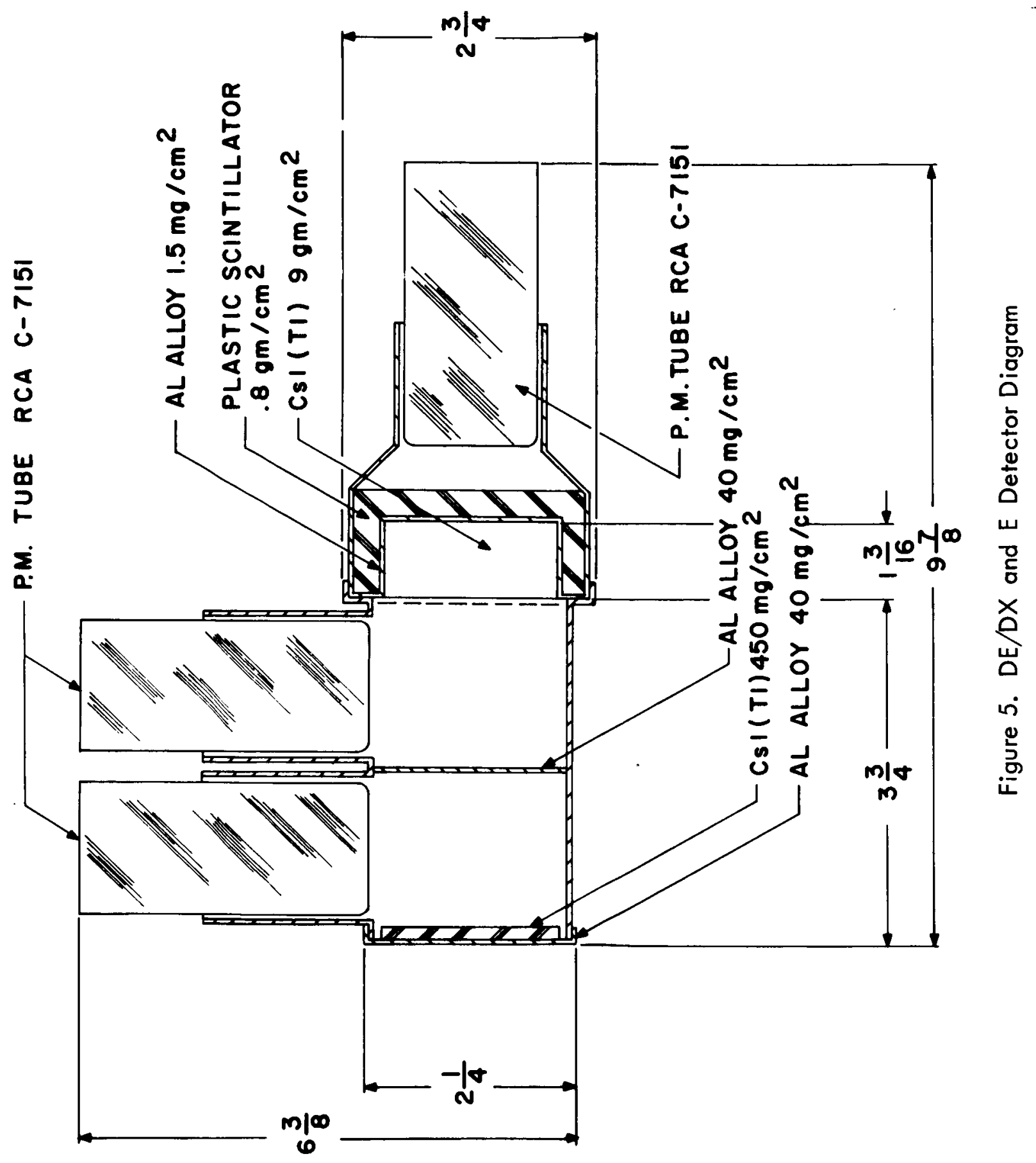




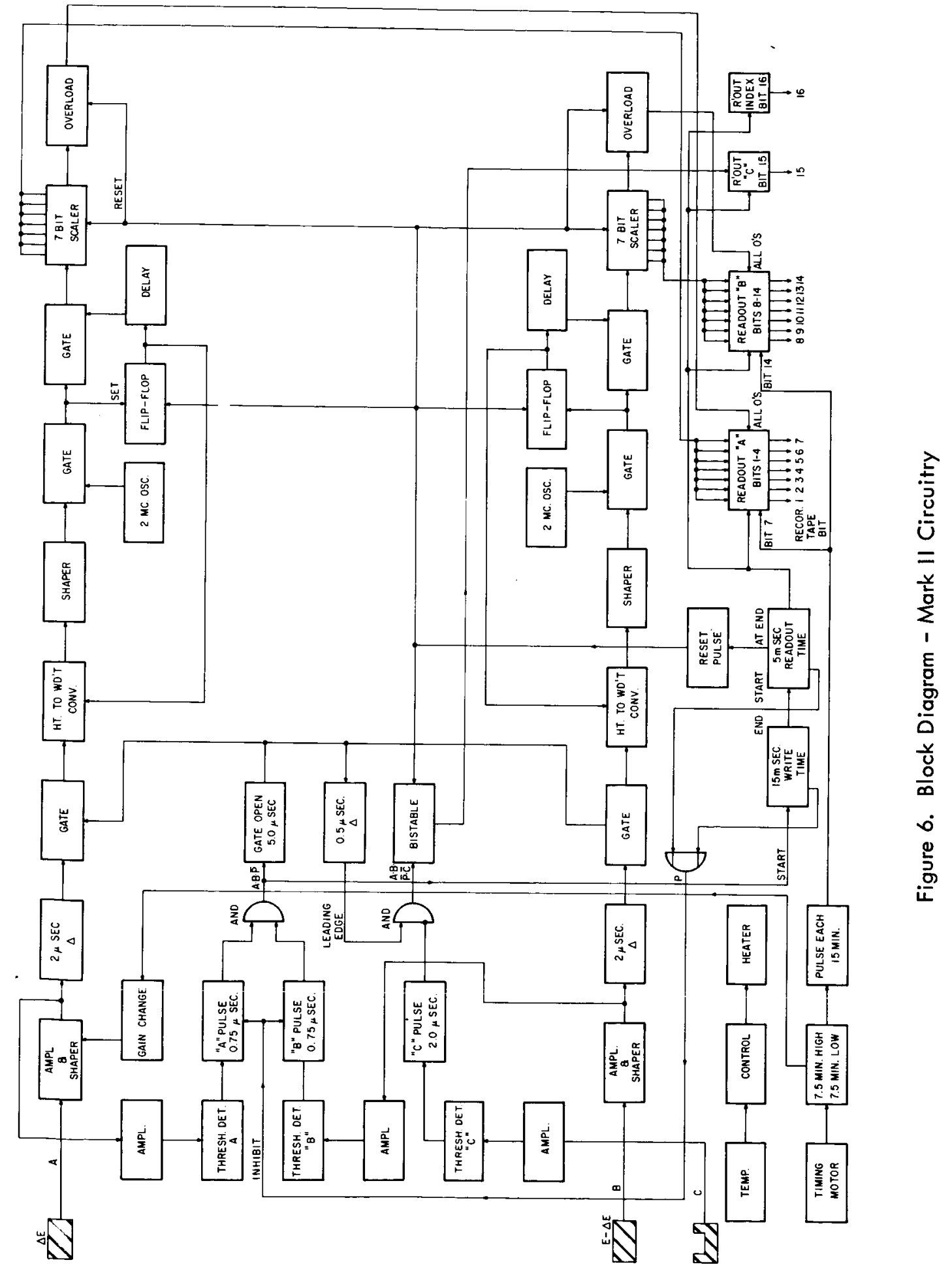




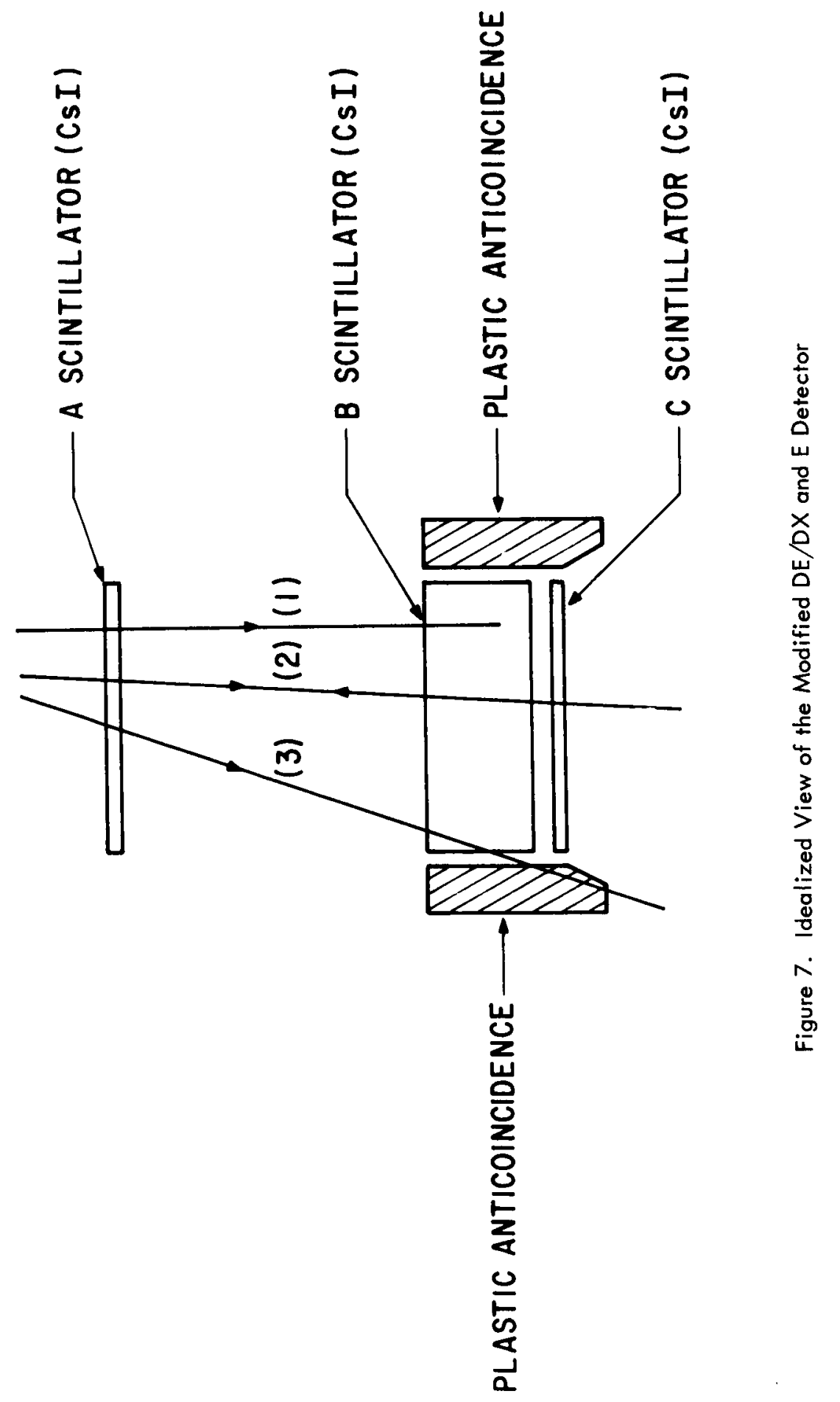




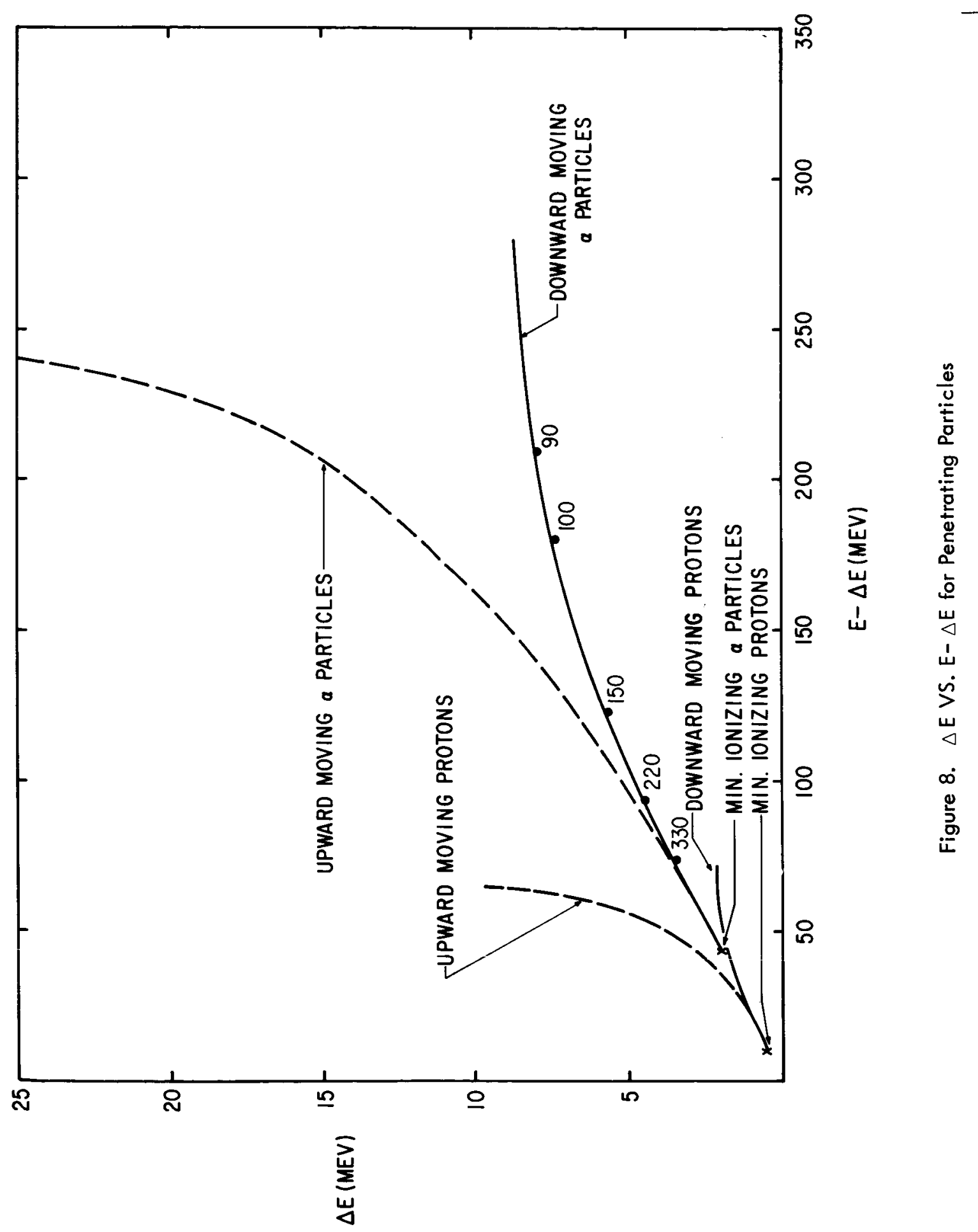




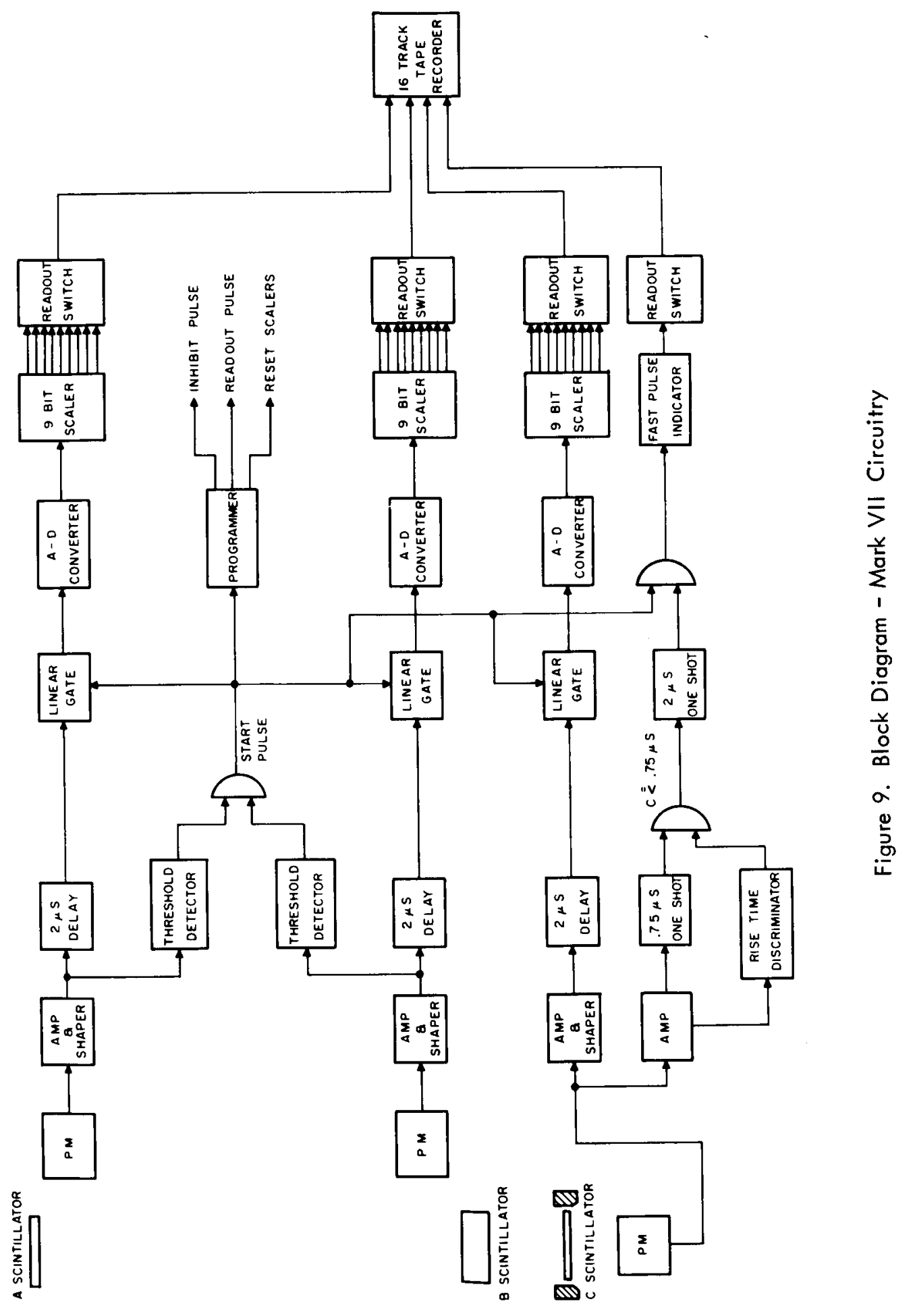




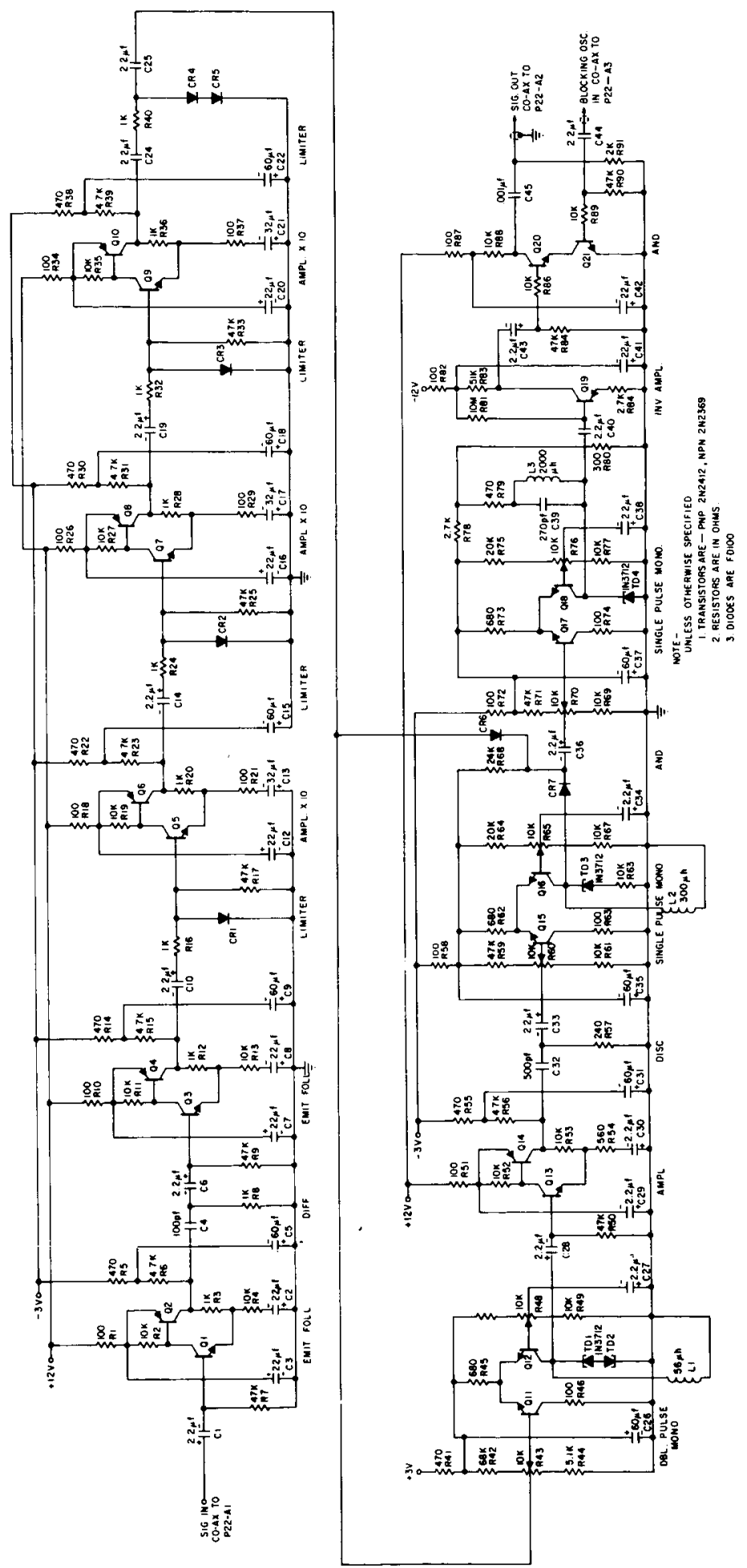

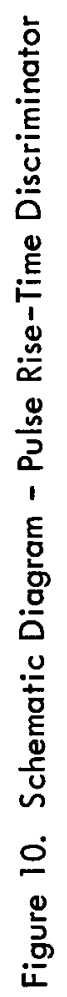




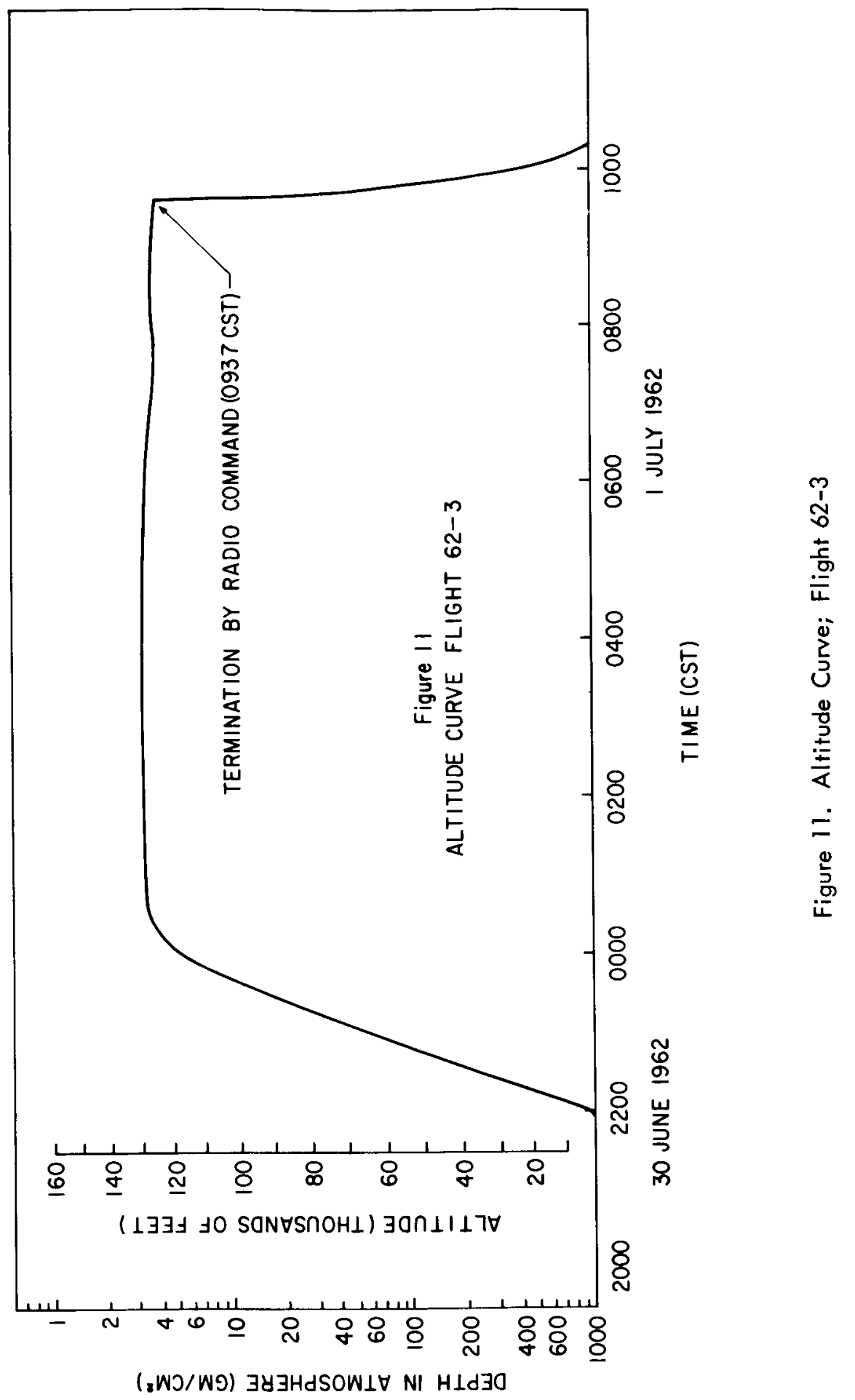




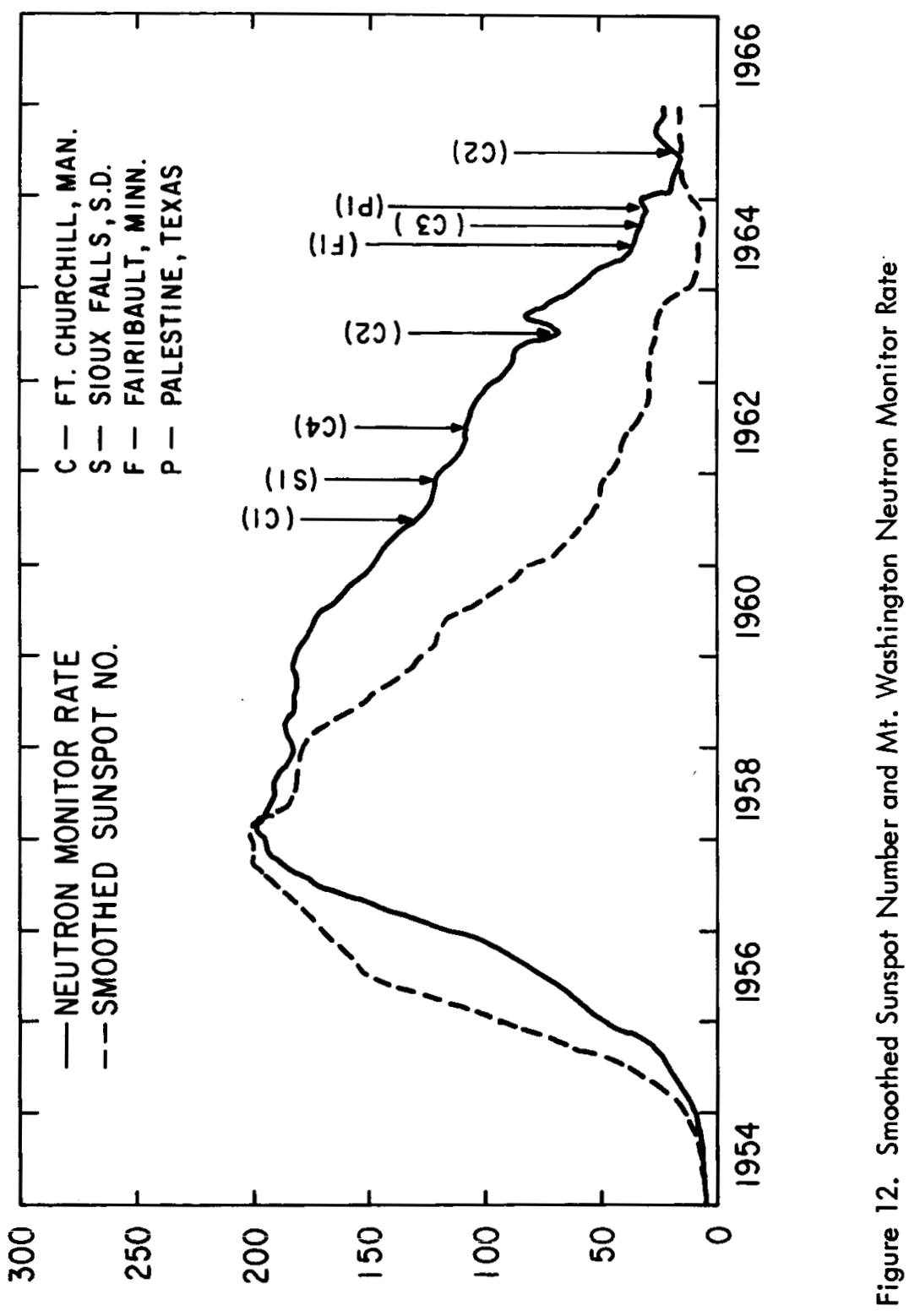

$\longleftarrow$ -

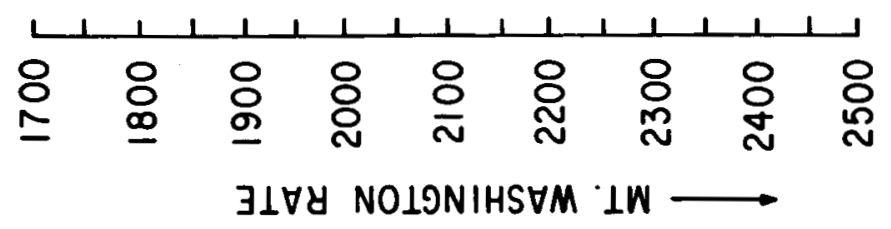




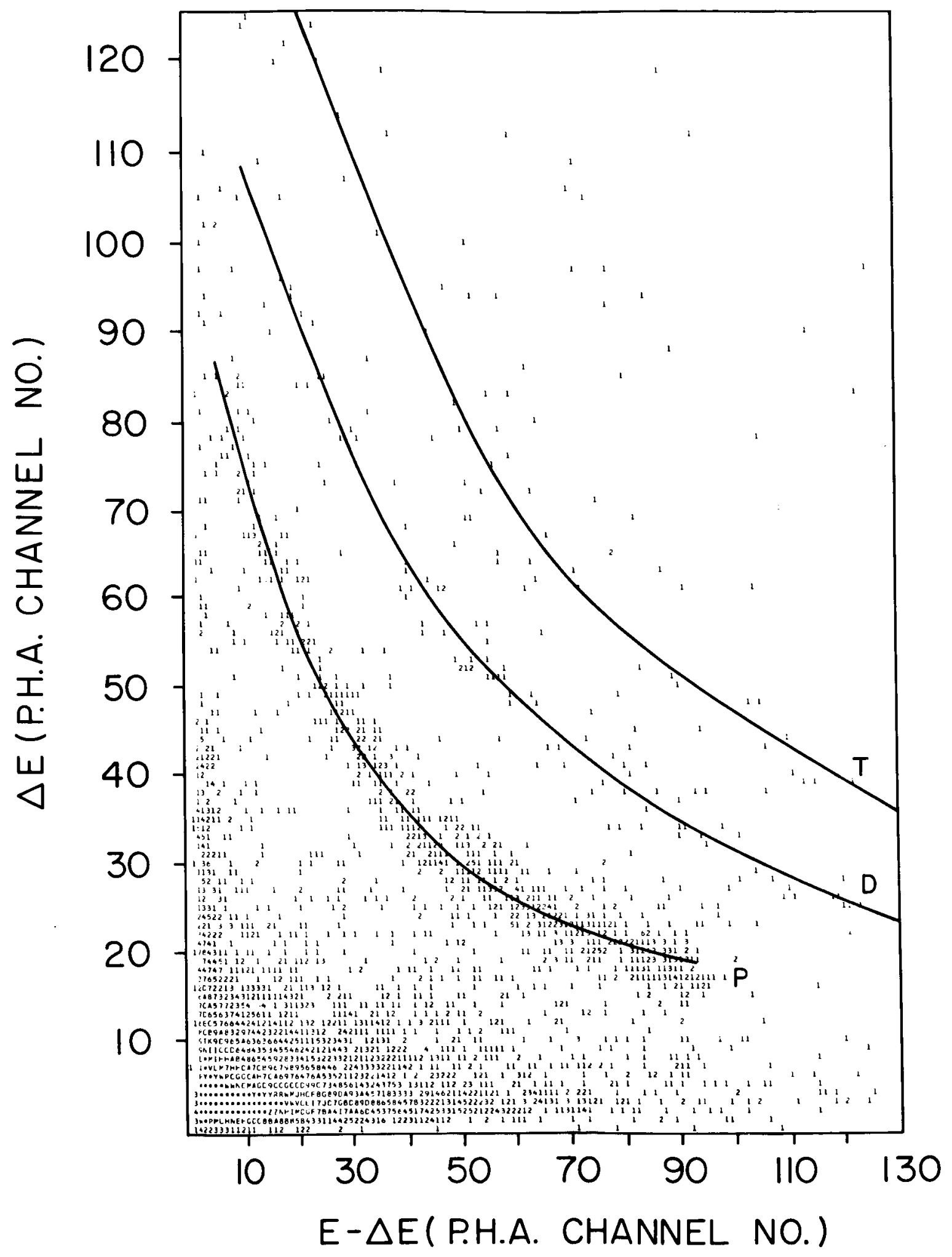

Figure 13. $\triangle E$ VS. E- $\triangle E$ (Stopping Particles); Flight $65-1 ; 128 \times 128$ Channels 


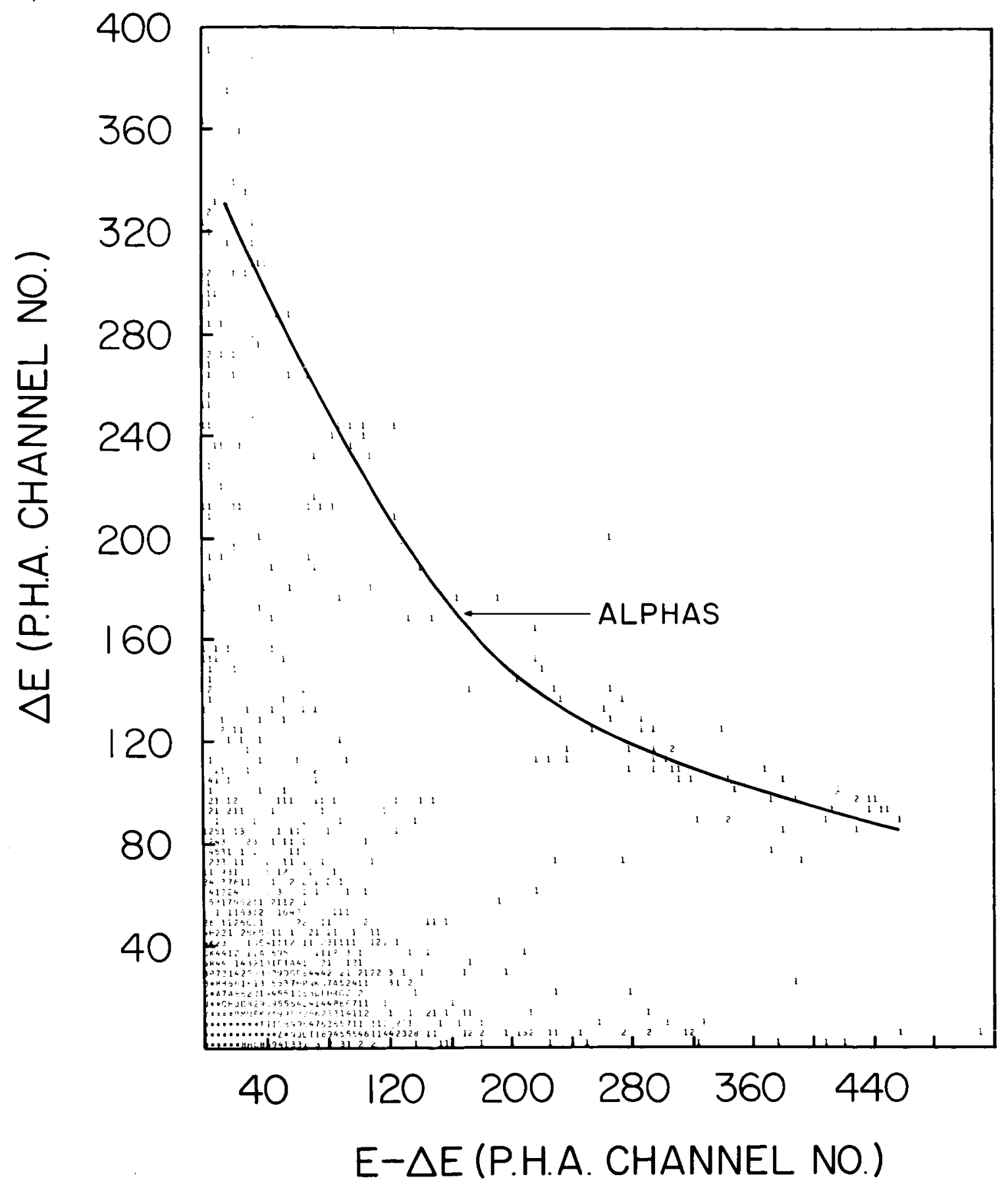

Figure 14. $\triangle E$ VS. E- $\triangle E$ (Stopping Particles); Flight $65-1 ; 512 \times 512$ Channels 


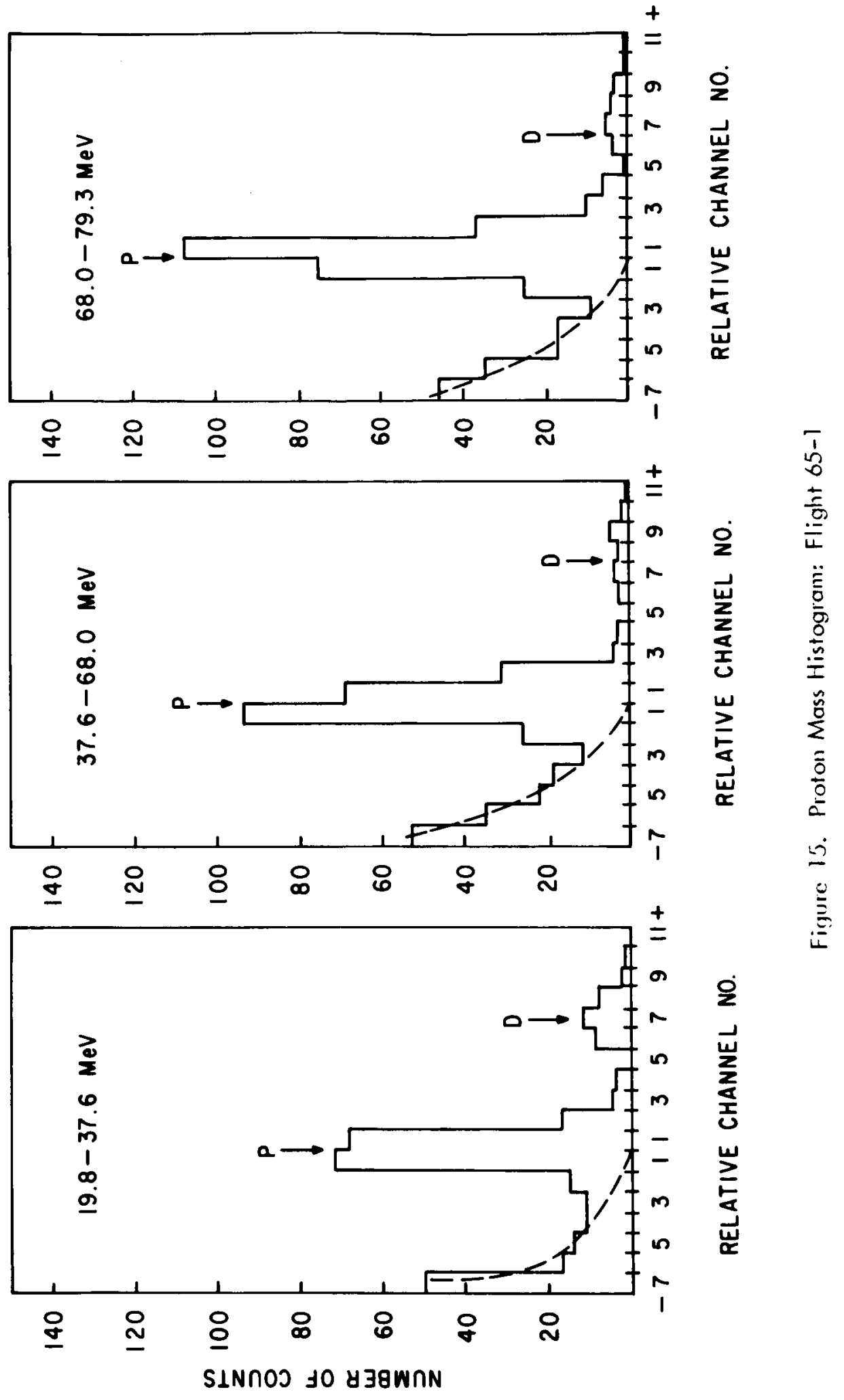




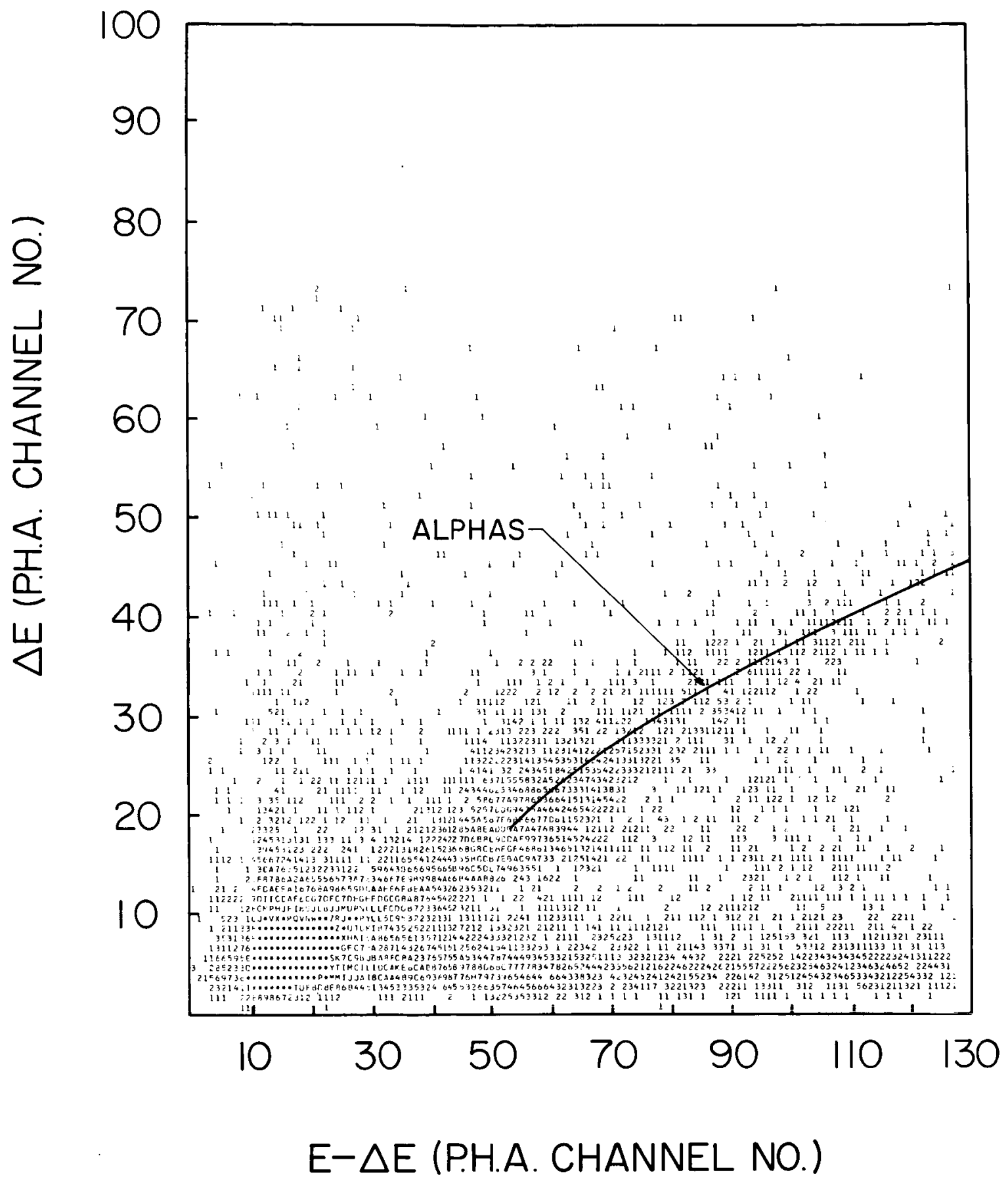

Figure 16. $\triangle E$ VS. E- $\triangle E$ (Penetrating Particles); Flight $65-1 ; 128 \times 128$ Channels 


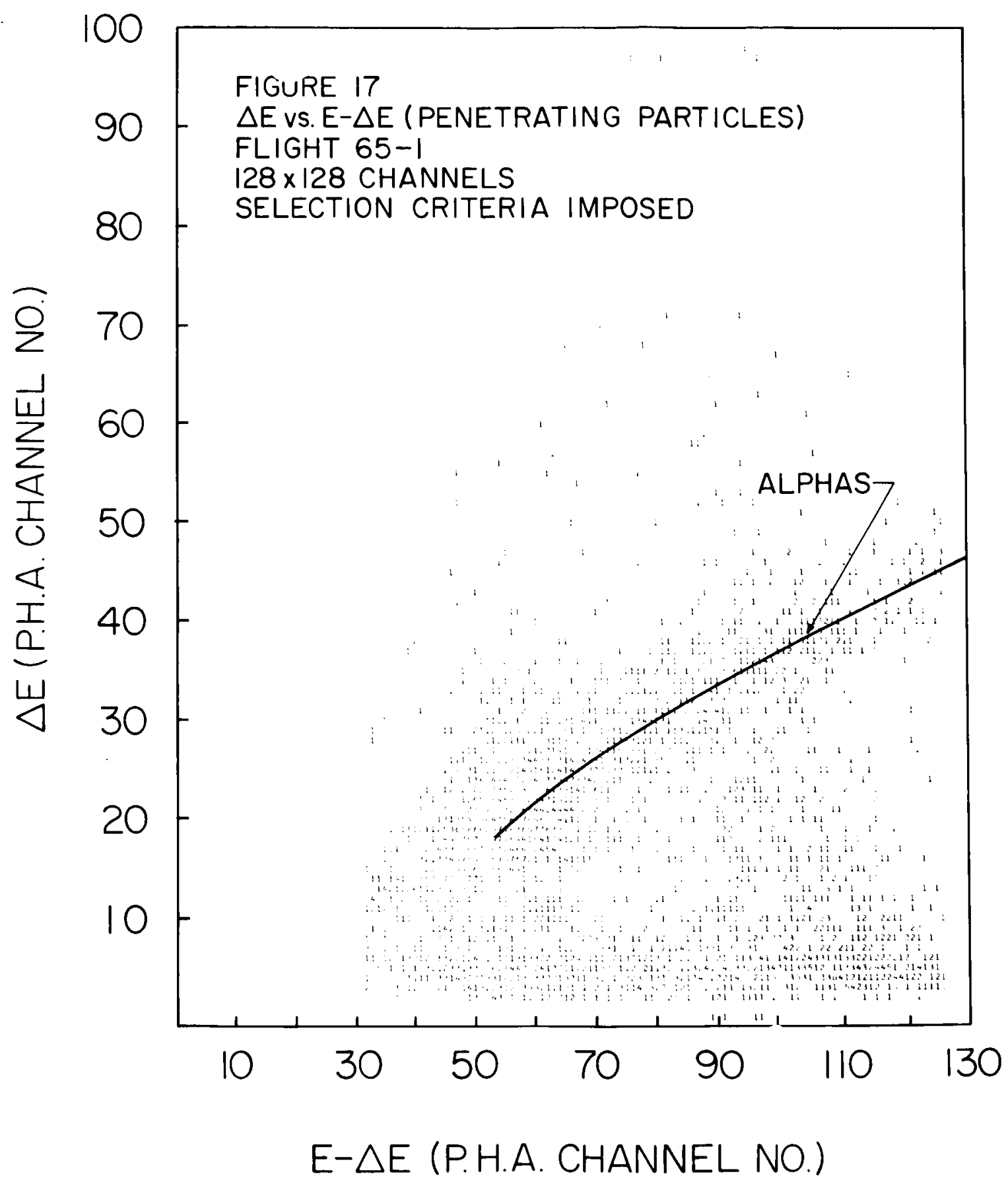

Figure 17. $\triangle E$ VS. E- $\triangle E$ (penetrating Particles); Flight $65-1 ; 128 \times 128$ Channels; Selection Criteria Imposed 


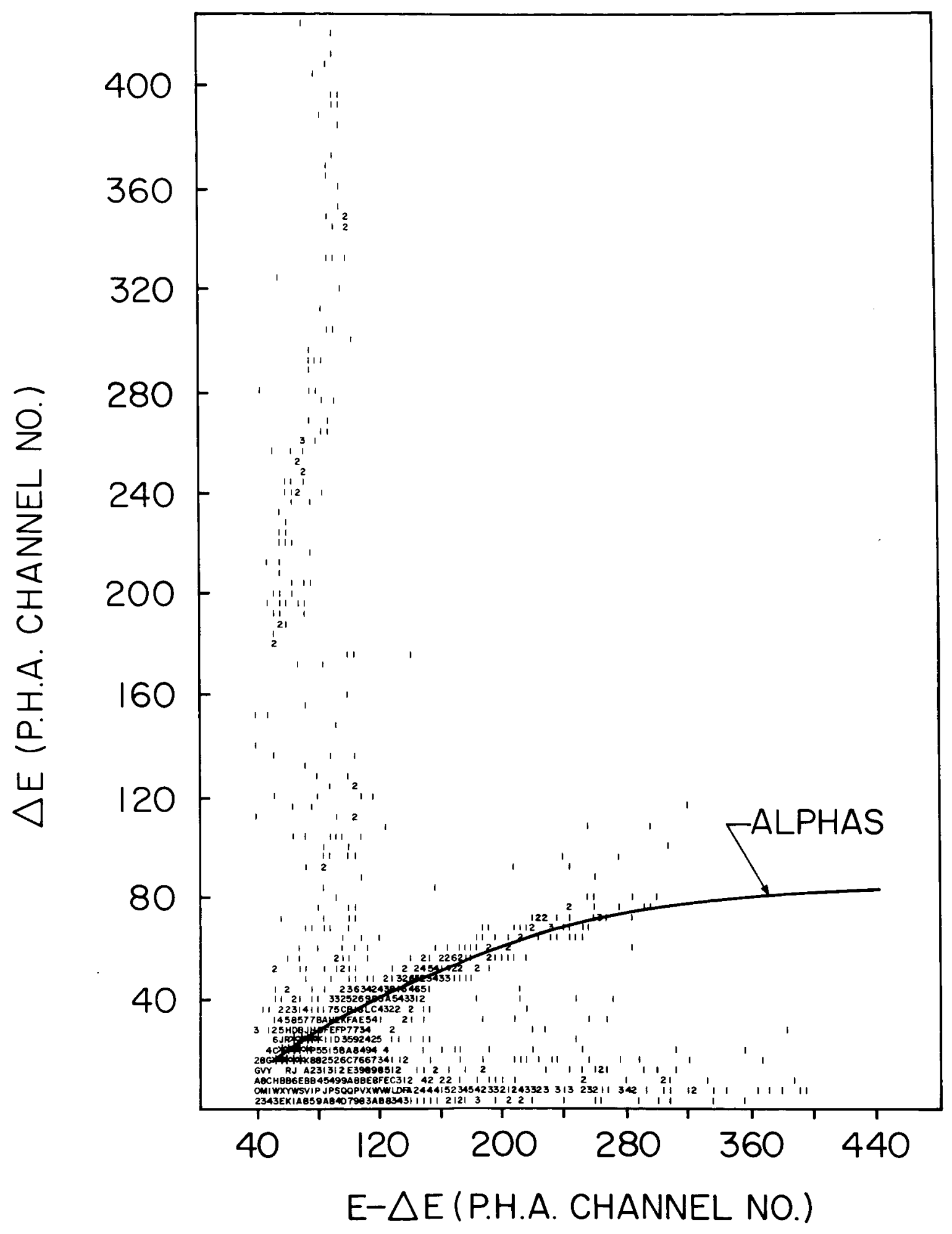

Figure 18. $\triangle E$ VS. $E-\triangle E$ (Penetrating Particles); Flight 65-1; $512 \times 512$ Channels; Selection Criteria Imposed 


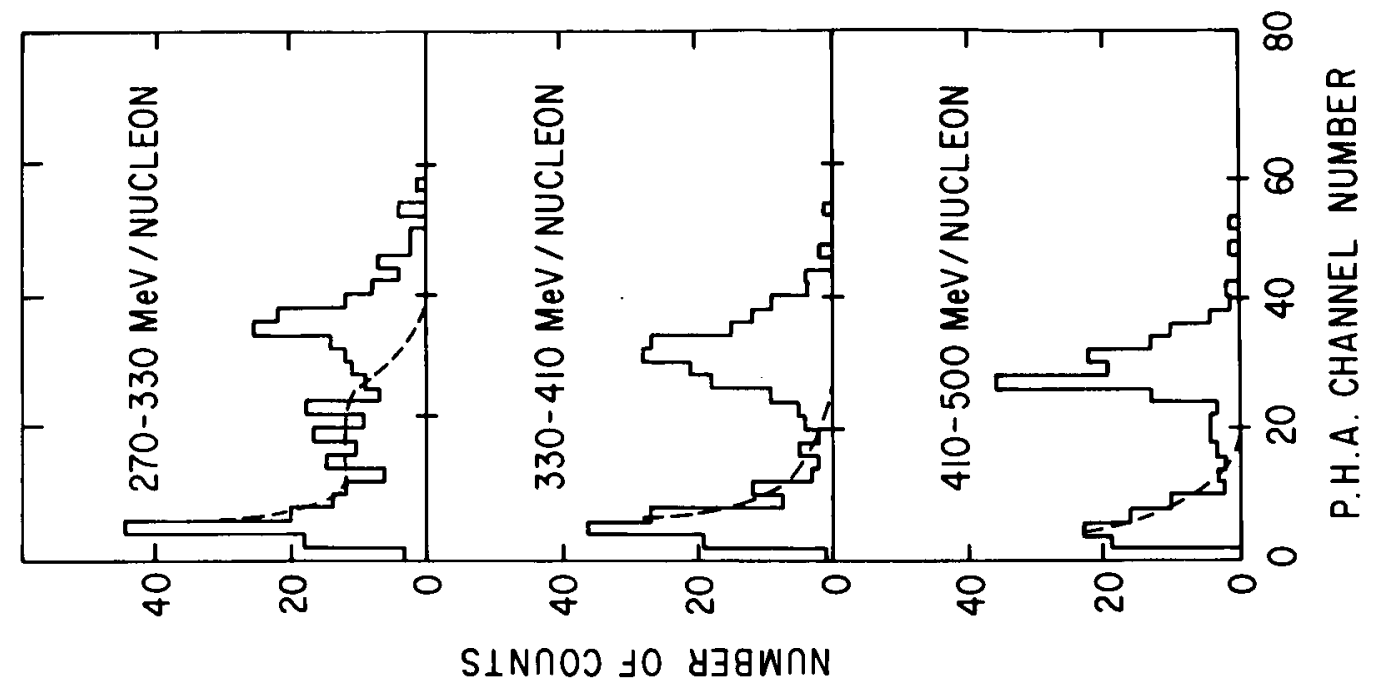

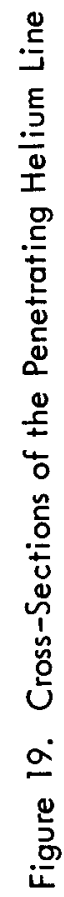

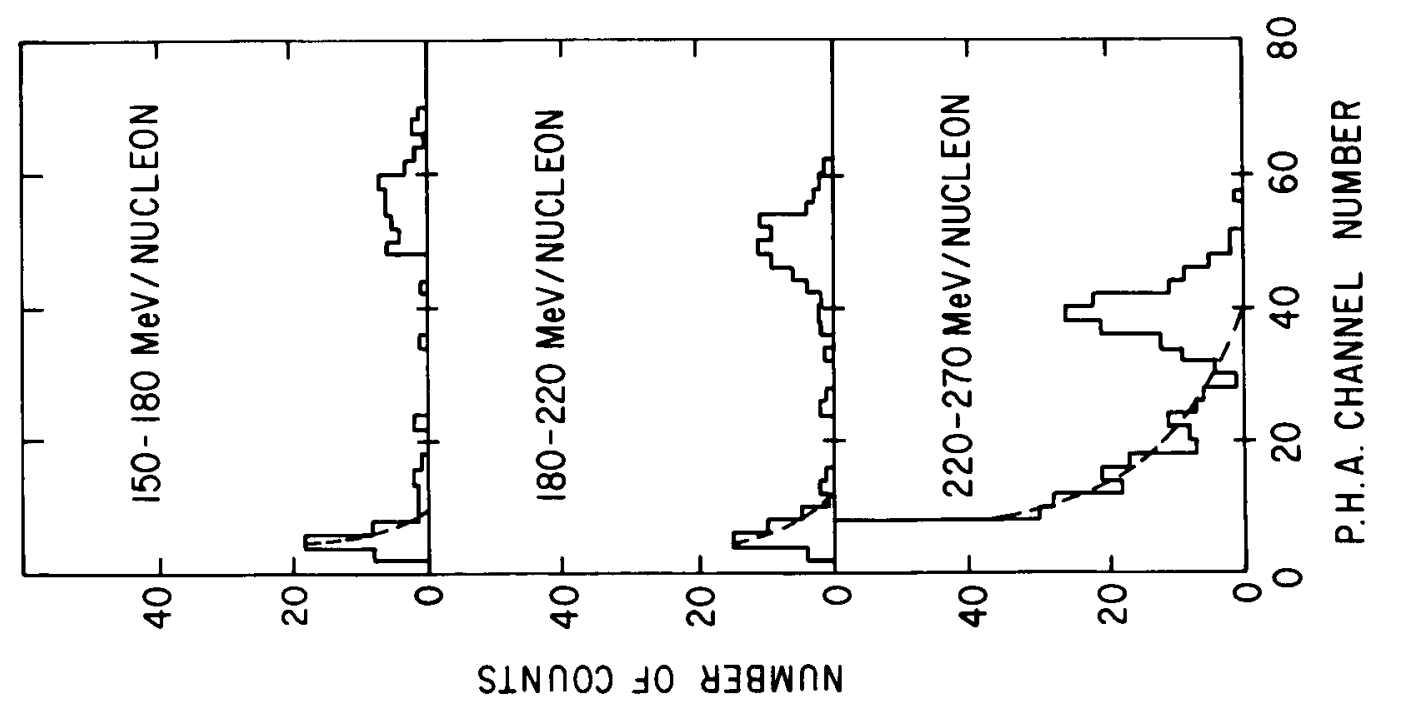




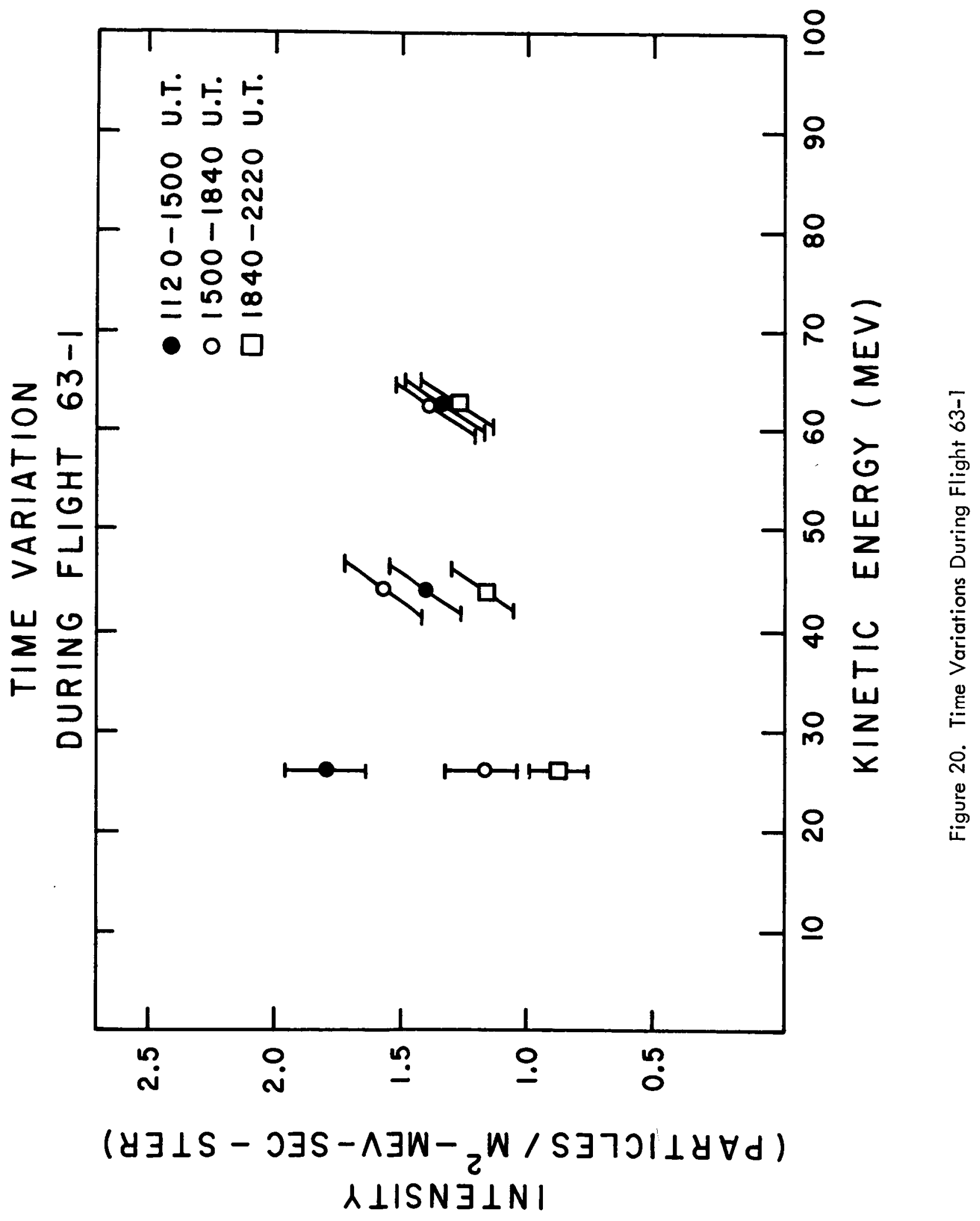




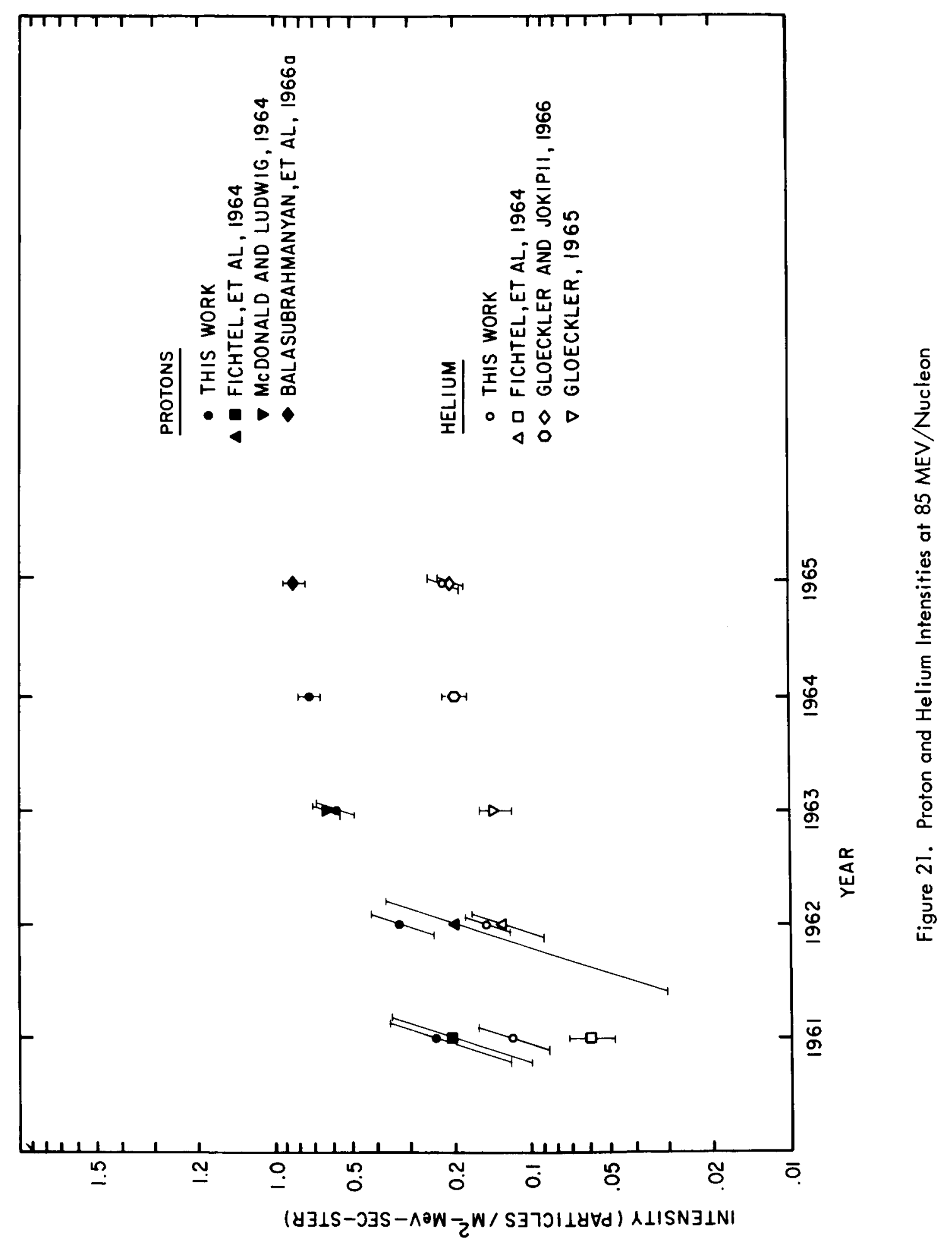




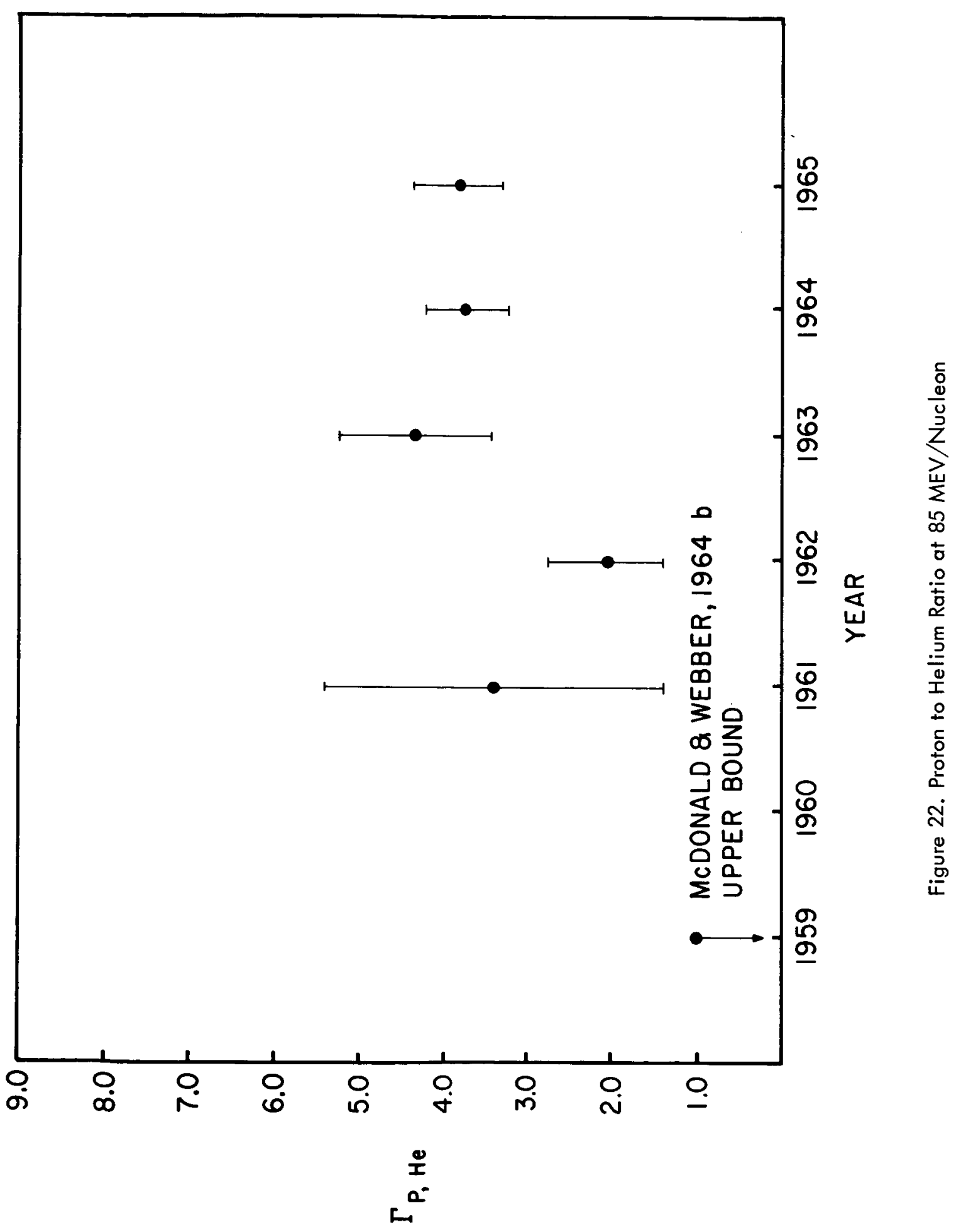




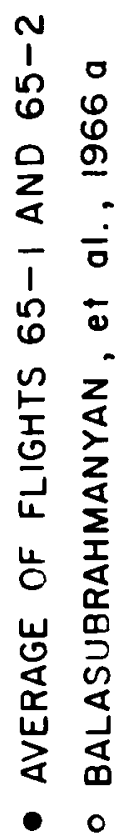

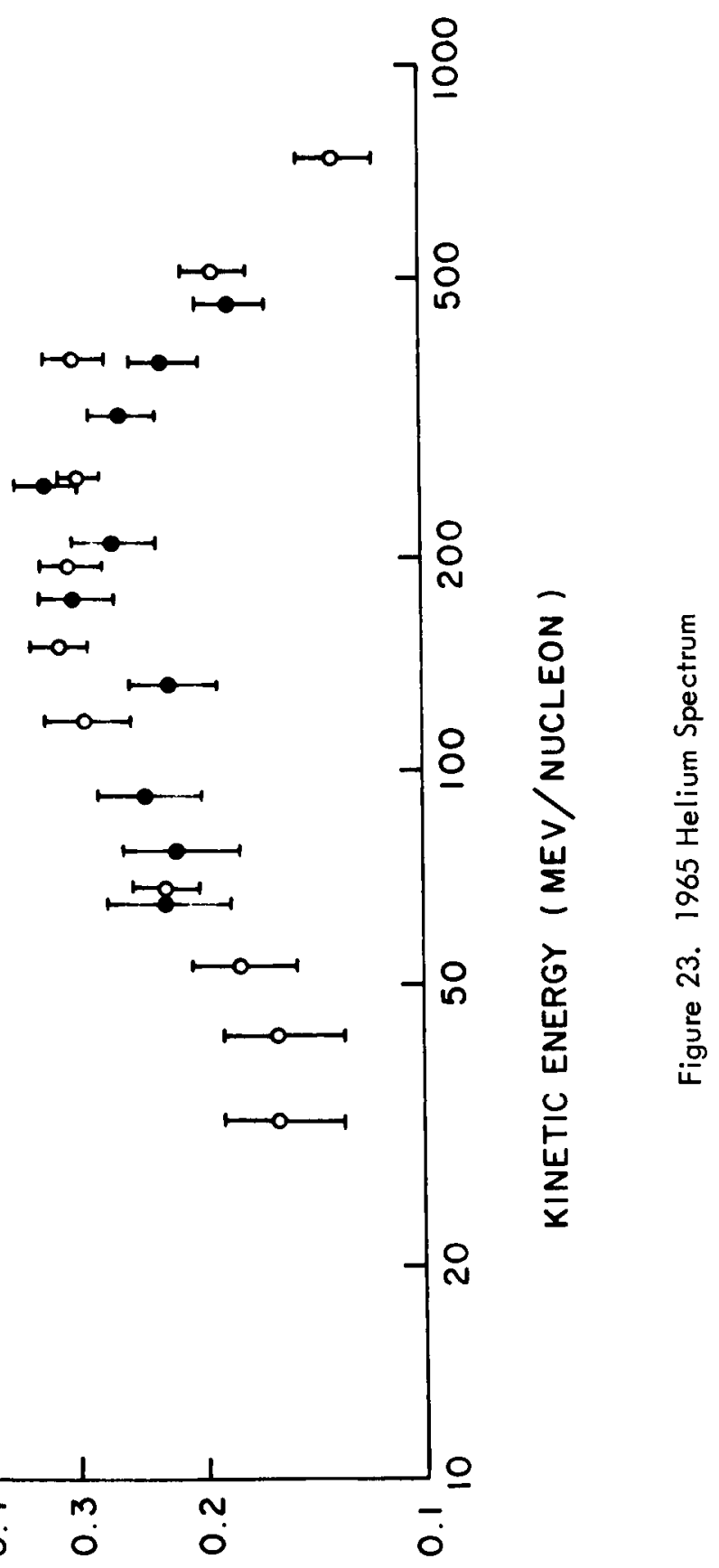

$($ INN/A3W-

$\downarrow \partial H+\varepsilon^{\partial H}$ 


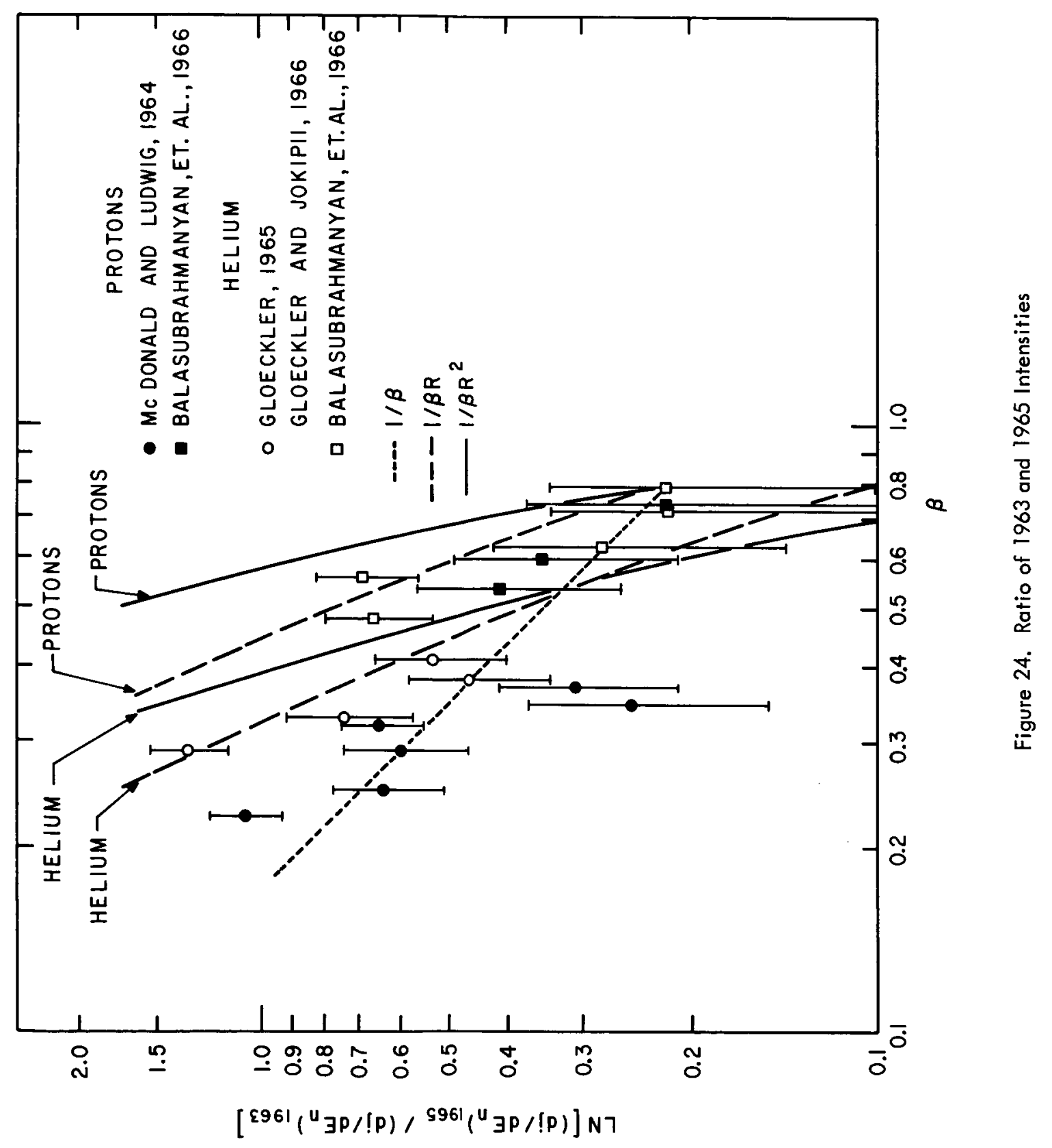




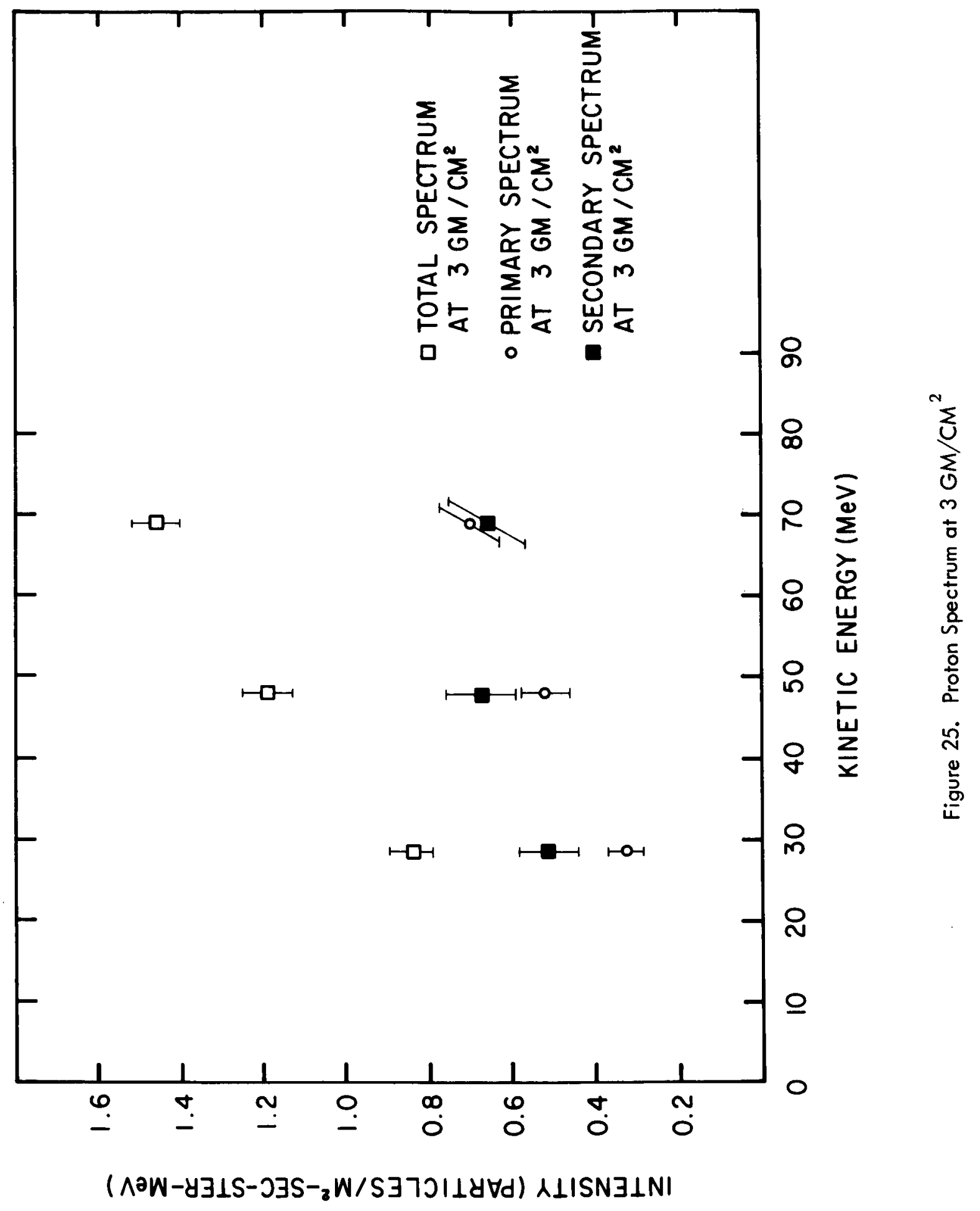




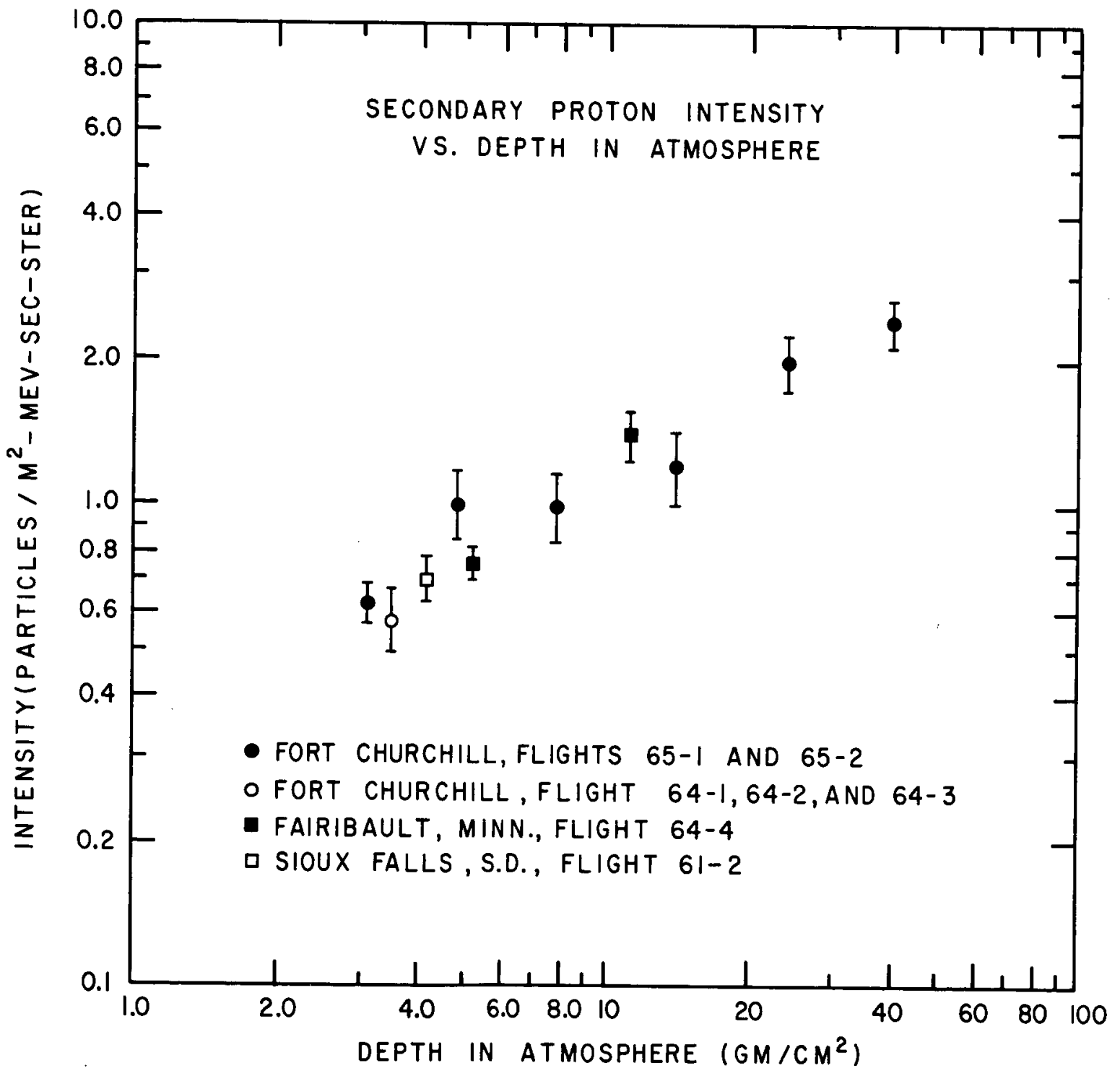

Figure 26. Secondary Proton Intensity VS. Depth in Atmosphere 


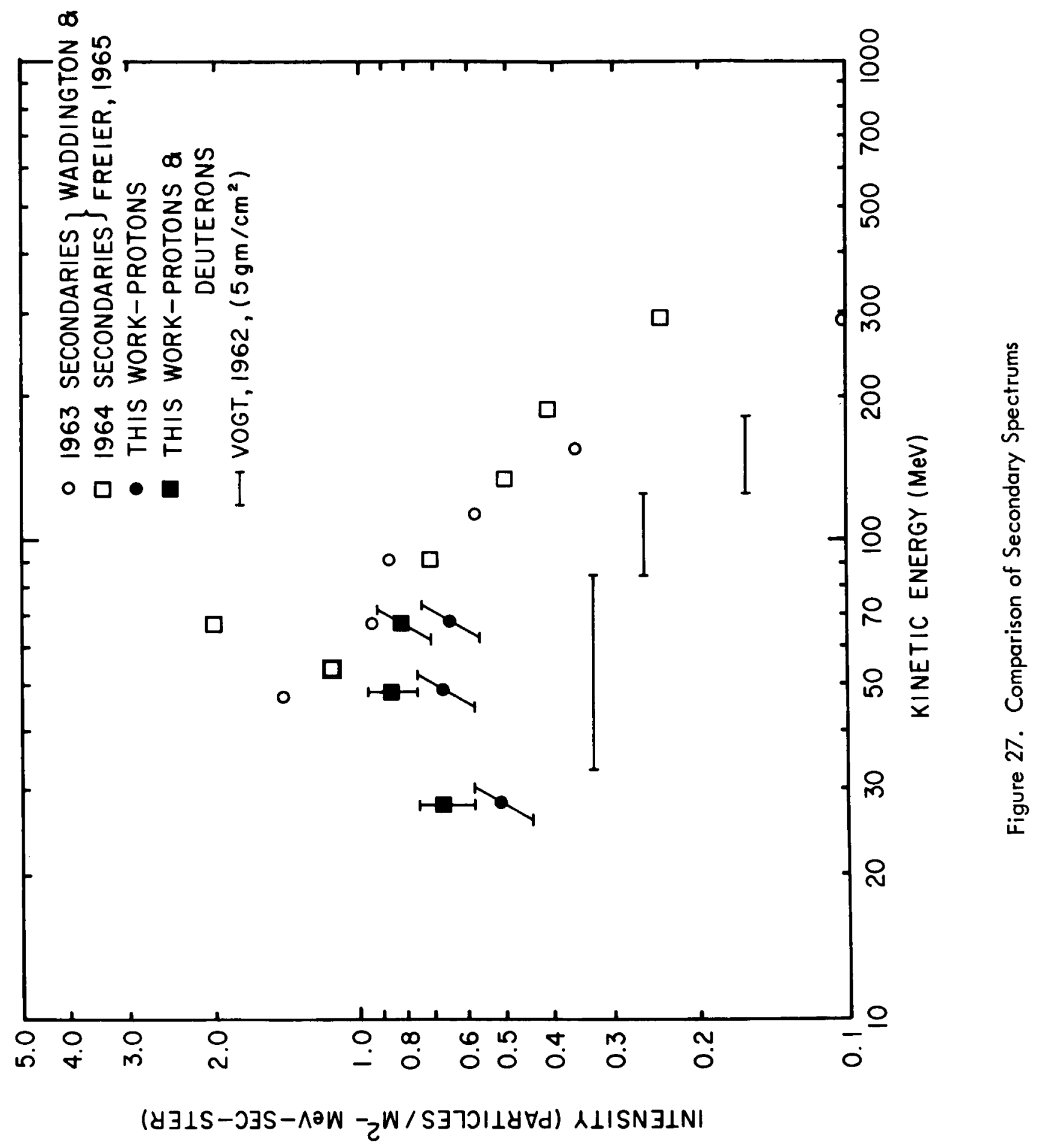




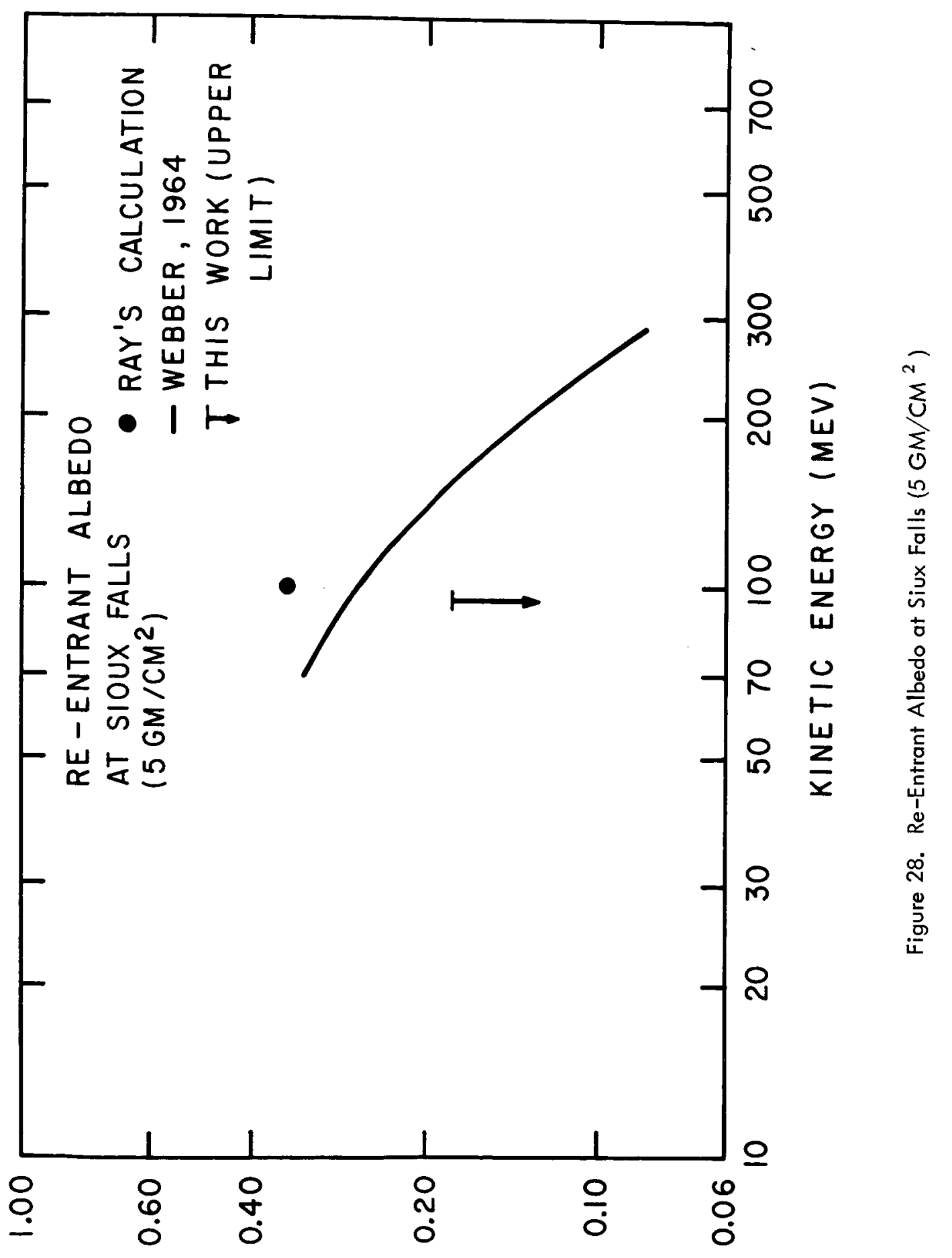

$\left(\forall \exists \perp S-\partial \exists S-\Lambda \exists W-{ }_{2} W / S \exists 7 J I \perp \forall \forall d\right) \wedge \perp I S N \exists \perp N \mid$ 

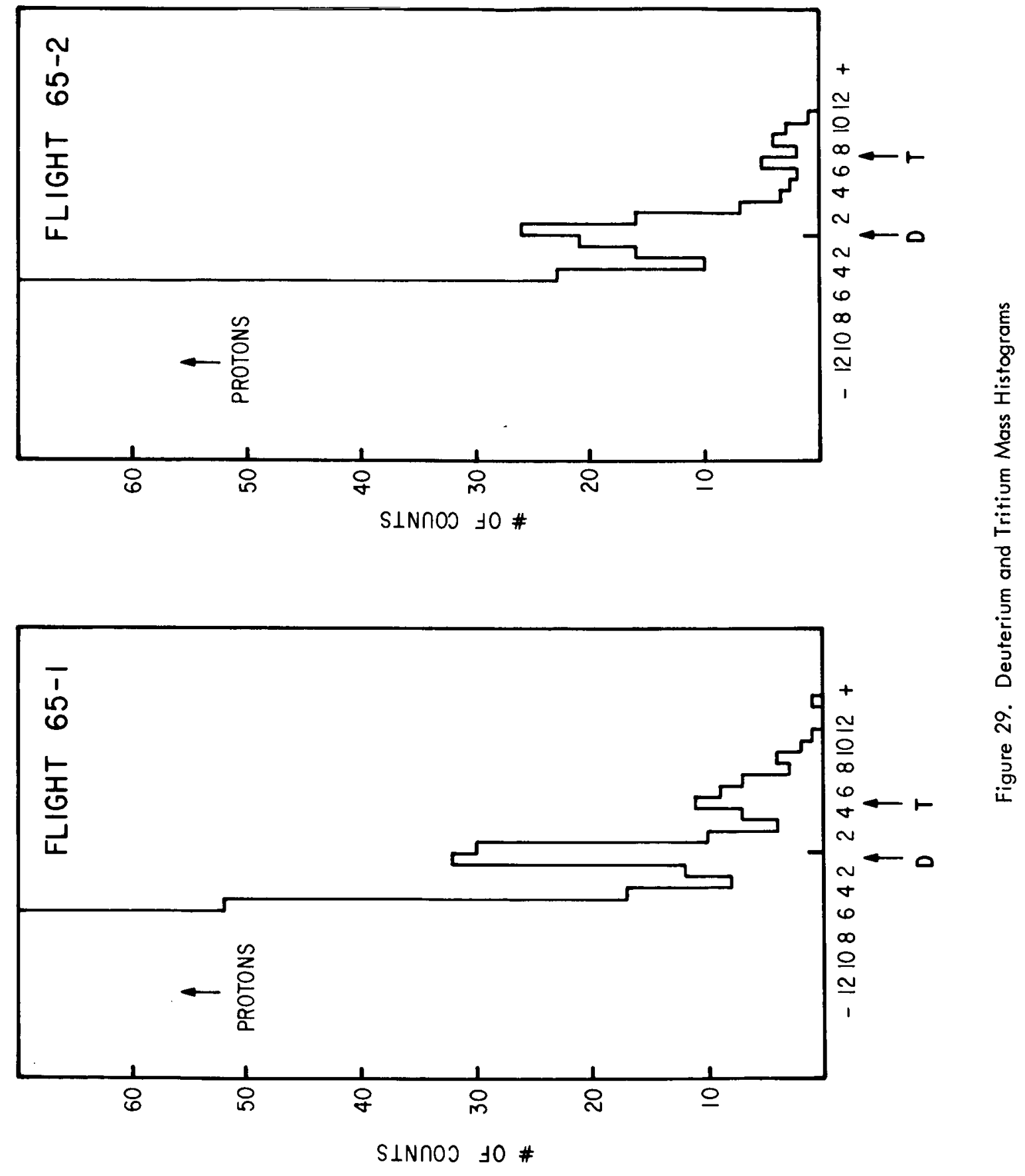


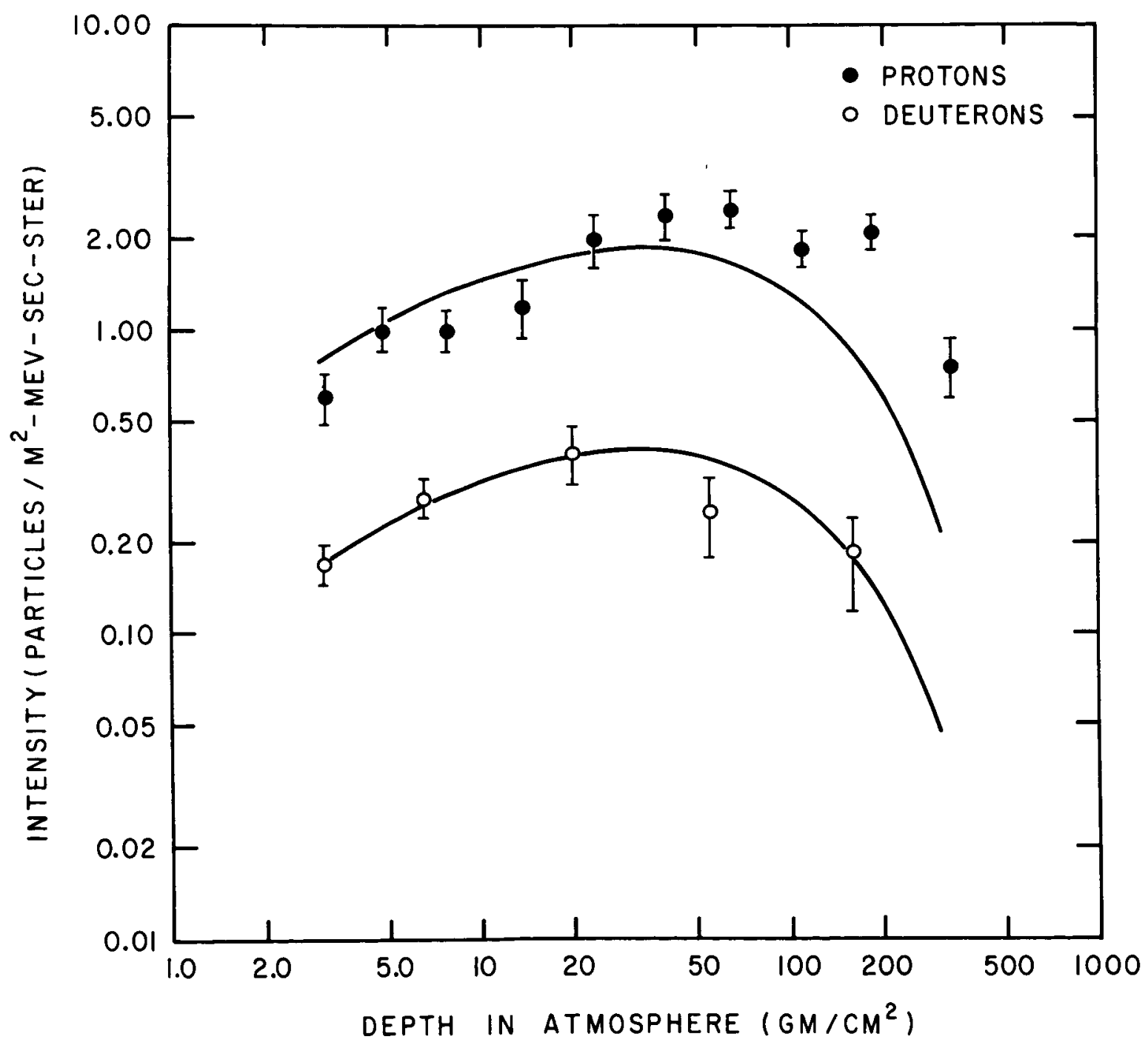

Figure 30. Secondary Proton and Deuteron Altitude Dependence 


\section{REFERENCES}

Anderson, H. R., Mariner 2: High Energy Radiation Experiment, Science, 139, Jan. 4, 1963.

Anderson, H. R., J. Geophys. Res., 69, 2561 (1964).

Balasubrahmanyan, V. K. , and McDonald, F. B., J. Geophys. Res., 69 , 3289 (1964).

Balasubrahmanyan, V. K., Brunstein, K.A., Ludwig, G. H., and McDonald, F. B., Transactions, American Geophys. Union, 46 (1), 124 (1965).

Balasubrahmanyan, V. K., Boldt, E., and Palmeira, R.A.R., J. Geophys. Res. (to be published).

Barkas, W. H., and Berger, M. J., NASA Publication SP-3013, 1964.

Benioff, P. A., Proceedings of Conference on Recent Research in Climatology, Ed. H. Craig, University of Calif., La Jolla, 1957 .

Bertini, H. W., Oak Ridge Nat. Lab. Tech. Report, ORNL-3383, Apr.,1963a.

Bertini, H. W., Phys. Rev., 131, 1801 (1963b).

Bertini, H. W., Oak Ridge Nat. Lab. Tech. Report, ORNL-3786, June, 1966.

Biswas, S., and Fichtel, C. E., Space Science Reviews, 4, 709 (1965).

Brunstein, K. A., Phys. Rev., 133, B1520 (1964).

Bryant, D. A., Cline, T. L., Desai, U. D., and McDonald, F. B., J. Geophys. Res., 67, 4983 (1962).

Bryant, D. A., Cline, T. L., Desai, U. D., and McDonald, F. B., Phys. Rev. Letters, 11,144 (1963).

Bryant, D. A., Cline, T. L., Desai, U. D., and McDonald, F. B., Ap. I., $\underline{141}, 478$ (1965).

Bryant, D. A., Cline, T. L., Desai, U. D., and McDonald, F. B., Phys. Rev. Letters, 14, 781 (1965).

Camerini, U., Davies, J. H., Fowler, P. H., Franzinetti, C. Muirhead, H., Lock, W. 0., Perkins, D. H., and Yekutieli, G., Phil. Mag., 42, 1241 (1951).

Courtier, G. M., and Linney, A. D., Proceedings International Conference on Cosmic Rays, London, p. 377, 1965.

Currie, L. A., Libby, W. F., and Wolfgang, R. L., Phys. Rev. 101, 1557 (1956). 
-109-

Dahanayake, C., Francois, P. E., Fujimoto, Y., Iredale, P., Waddington, C. J., and Yasin, M., Nuovo Cimento 1,888 (1955).

Davis, L. R., Caulk, H. M., and Johnson, C. Y., Phys. Rev., 101, 801 (1956).

Dorman, L. I., Proceedings of the Moscow Cosmic Ray Conference, Vo1. IV, p. 320, International Union of Pure and Applied Physics, Moscow, 1960 .

Dorman, L. I., Progress in Elementary Particles and Cosmic Ray Physics, Vo1. VII, P. 1, North-Holland Publishing Co., Amsterdam, 1963.

Duke, P. J., Phil. Mag., 5, 1151 (1960).

Fan, C. Y., Gloeckler, G., and Simpson, J. A., Trans. American Geophys. Union, 45 (3), 507 (1964).

Fan, C. Y., Gloeckler, G., and Simpson, J. A., J. Geophys. Res., 70, 3515 , (1965a).

Fan, C. Y., Gloeckler, G., and Simpson, J. A., Trans. American Geophys. Union, 46 (1), 1246 (1965b).

Fan, C. Y., Gloeckler, G., and Simpson, J. A., Phys. Rev. Letters, 17, 329 (1966).

Fichte1, C. E., Guss, D. E., Kniffen, D. A., and Neelakantan, J., J. Geophys. Res., 3293 (1964).

Fichte1, C. E., Durgaprasad, N., and Guss, D. E., Proceedings Internationa1 Conference on Cosmic Rays, London, p. 371, 1965.

Fireman, E. L., and Rowland, F. S., Phys. Rev., 97, 780 (1955).

Forbush, S. E., J. Geophys. Res., 59, 525 (1954).

Fowler, P. H., Freier, P. S., and Ney, E. P., Nature, 181, 1319 (1958).

Freier, P. S., Ney, E. P., and Waddington, C. J., Phys. Rev., 114, 365 (1959).

Freier, P. S., and Waddington, C. J., Phys. Rev. Letters, 13, 108 (1964).

Freier, P. S., and Waddington, C. J., Univ. of Minn. Tech. Report CR-74, 1965 .

Freier, P. S., and Waddington, C. J., J. Geophys. Res., 70, 5753 (1965).

Gloeckler, G., J. Geophys. Res., 70, 5333 (1965).

Gloeckler, G., and Jokipii, J. R., Phys. Rev. Letters, 17, 203 (1966). 
Hagge, Donald E., Ludwig, George H., and McDonald, Frank B., paper presented at American Physical Society Meeting, Washington, D.C., April, 1966.

Lal, D., and Peters, B., Progress in Elementary Particles and Cosmic Ray Physics, Vol. VI, p. 1, North-Holland Publishing Co., Amsterdam, 1962 .

Maehlum, B., and O'Brien, B. J., J. Geophys. Res., 67, 3269 (1962).

McDonald, F. B., and Webber, W. R., Phys. Rev., 104, 1723 (1956).

McDonald, F. B., and Webber, W. R., Phys. Rev., 107, 1386 (1957).

McDonald, F. B., and Webber, W. R., Phys. Rev., 115, 194 (1959).

McDonald, F. B., and Webber, W. R., J. Geophys. Res. 67, 2119 (1962).

McDonald, F. B., and Ludwig, G. H., Phys. Rev. Letters, 13, 783. (1964).

Metropolis, N., Bivins, R., Storm, M., Turkevich, Anthony, Miller, J. M. and Friedlander, G., Phys. Rev., 110, 185 (1958).

Metropolis, N., Bivins, R., Storm, M., Miller, J. M., Friedlander, G., and Turkevich, Anthony, Phys. Rev., 110, 204 (1958b).

Meredith, L. H., Van Allen, J. A., and Gottlieb, M. B., Phys. Rev., 99, 199 (1955).

Meyer, P., and Simpson, J. A., Phys. Rev., 99, 1517 (1955).

Meyer, P., and Simpson, J. A., Phys. Rev., 106, 568 (1957).

Meyer, P., and Vogt, R., Phys. Rev., 129, 2275 (1963).

Neher, H. V., Phys. Rev., 103, 228 (1956).

Neher, H. V., Phys. Rev., 107, 588 (1957).

Neher, H. V., and Anderson, H. R., J. Geophys. Res., 69, 807 (1964).

Ness, N. F., Scearce, C. F., and Seek, J. B., J. Geophys. Res., 69, 3531 (1964).

Ney, E. P., Proceedings of Internationa1 Conference on Cosmic Rays, Guanajuato, 1955 .

Ormes, J., and Webber, W. R. Proceedings International Conference on Cosmic Rays, Jaipur, 1963.

Ormes, J., and Webber, W. R., Phys. Rev. Letters, 13, 106 (1964). 
Powe11, C. F., Fowler, P. H., and Perkins, D. H., The Study of Elementary Particles by the Photographic Method, Pergamon Press, New York, 1959.

Ray, E. C., An Improved Reentrant Cosmic Ray Albedo Calculation, (to be published).

Shea, M. A., and Smart, D. F., Cosmic Ray Cutoff Rigidity Calculations and their Application to Experimental Measurements, "paper presented at Symposium on Recent Advances in Cosmic Ray Research, Douglas Advanced Research Laboratories, Huntingdon Beach, Calif., April, 1966.

Silberberg, R., Phys. Rev., 148, 1247 (1966).

Simpson, J. A., J. Geophys. Res., 65, 1615, (1960).

Singer, S. F., Progress in Elementary Particles and Cosmic Ray Physics, Vo1. IV, p. 205, North-Holland Publishing Co., Amsterdam, 1958.

Vogt, R., Phys. Rev., 125, 366 (1962).

Waddington, C. J., Phil. Mag., 45, 1312 (1954).

Waddington, C. J., Progress in Nuclear Physics, Vol. 8, p. 3, Pergamon Press, New York, 1960.

Waddington, C. J., and Freier, P. S., Proceedings International Conference on Cosmic Rays, London, p. 339, 1965.

Webber, W. R., and McDonald, F. B., Phys. Rev., 100, 1460 (1955).

Webber, W. R., Progress in Elementary Particles and Cosmic Ray Physics, Vo1. VI, p. 77, North-Holland Pub1ishing Co., Amsterdam, 1962.

Webber, W. R., and Ormes, J. F., Proceedings of International Conference on Cosmic Rays, Jaipur, 1963.

Webber, W. R., The Spectrum and Charge Composition of the Primary Cosmic Radiation, Univ. of Minn. Technical Report CR -76, Dec., $1964 \mathrm{a}$.

Webber, W. R., and McDonald, F. B., J. Geophys. Res., 69, 3097 (1964b).

Webber, W. R., Proceedings of International Conference on Cosmic Rays, London, p. 345,1965 .

Winckler, J. R., and Anderson, K. A., Phys. Rev., 108, 148 (1957). 